\title{
DERIVED EQUIVALENCES OF K3 SURFACES AND ORIENTATION
}

\author{
DANIEL HUYBRECHTS, EMANUELE MACRÌ, AND PAOLO STELLARI
}

\begin{abstract}
Every Fourier-Mukai equivalence between the derived categories of two K3 surfaces induces a Hodge isometry of their cohomologies viewed as Hodge structures of weight two endowed with the Mukai pairing. We prove that this Hodge isometry preserves the natural orientation of the four positive directions. This leads to a complete description of the action of the group of all autoequivalences on cohomology very much like the classical Torelli theorem for K3 surfaces determining all Hodge isometries that are induced by automorphisms.
\end{abstract}

\section{INTRODUCTION}

The second cohomology $H^{2}(X, \mathbb{Z})$ of a $\mathrm{K} 3$ surface $X$ is an even unimodular lattice of signature $(3,19)$ endowed with a natural weight two Hodge structure. The inequality $(\alpha, \alpha)>0$ describes an open subset of the 20-dimensional real vector space $H^{1,1}(X) \cap H^{2}(X, \mathbb{R})$ with two connected components $\mathcal{C}_{X}$ and $-\mathcal{C}_{X}$. Here $\mathcal{C}_{X}$ denotes the positive cone, i.e. the connected component that contains the Kähler cone $\mathcal{K}_{X}$ of all Kähler classes on $X$.

Any automorphism $f: X \stackrel{\sim}{\longrightarrow} X$ of the complex surface $X$ defines an isometry

$$
f_{*}: H^{2}(X, \mathbb{Z}) \stackrel{\sim}{\longrightarrow} H^{2}(X, \mathbb{Z})
$$

compatible with the weight two Hodge structure. In particular, $f_{*}$ preserves the set $\mathcal{C}_{X} \sqcup\left(-\mathcal{C}_{X}\right)$. As the image of a Kähler class is again a Kähler class, one actually has $f_{*}\left(\mathcal{C}_{X}\right)=\mathcal{C}_{X}$. In other words, $f_{*}$ respects the connected components of the set of $(1,1)$-classes $\alpha$ with $(\alpha, \alpha)>0$. If one wants to avoid the existence of Kähler structures, the proof of this assertion is a little more delicate. However, applying his polynomial invariants, Donaldson proved in [11] a much stronger result not appealing to the complex or Kähler structure of $X$ at all.

Before recalling his result, let us rephrase the above discussion in terms of orientations of positive three-spaces. Consider any three-dimensional subspace $F \subset H^{2}(X, \mathbb{R})$ on which the intersection pairing is positive definite. Then $F$ is called a positive three-space. Using orthogonal projections, given orientations on two positive three-spaces can be compared to each other. So, if $\rho$ is an arbitrary isometry of $H^{2}(X, \mathbb{R})$ and $F$ is a positive three-space, one can ask whether a given orientation of $F$ coincides with the image of this orientation on $\rho(F)$. If this is the case, then one says that $\rho$ is orientation preserving. Note that this does neither depend on $F$ nor on the chosen orientation of $F$. The fact that any automorphism $f$ of the complex surface $X$ induces a Hodge isometry with $f_{*}\left(\mathcal{C}_{X}\right)=\mathcal{C}_{X}$ is equivalent to saying that $f_{*}$ is orientation preserving. More generally one has:

Theorem 1. (Donaldson) Let $f: X \stackrel{\sim}{\rightarrow} X$ be any diffeomorphism. Then the induced isometry $f_{*}: H^{2}(X, \mathbb{Z}) \stackrel{\sim}{\longrightarrow} H^{2}(X, \mathbb{Z})$ is orientation preserving.

This leads to a complete description of the image of the natural representation

$$
\operatorname{Diff}(X) \longrightarrow \mathrm{O}\left(H^{2}(X, \mathbb{Z})\right)
$$

2000 Mathematics Subject Classification. 18E30, 14J28.

Key words and phrases. K3 surfaces, derived categories, deformations. 
as the set of all orientation preserving isometries of the lattice $H^{2}(X, \mathbb{Z})$. That every orientation preserving isometry can be lifted to a diffeomorphism relies on the Global Torelli theorem (see [2]). The other inclusion is the above result of Donaldson.

There are several reasons to pass from automorphisms or, more generally, diffeomorphisms of a K3 surface $X$ to derived autoequivalences. First of all, exact autoequivalences of the bounded derived category

$$
\mathrm{D}^{\mathrm{b}}(X):=\mathrm{D}^{\mathrm{b}}(\mathbf{C o h}(X)) \simeq \mathrm{D}_{\text {coh }}^{\mathrm{b}}\left(\mathcal{O}_{X}-\mathbf{M o d}\right)
$$

of coherent sheaves can be considered as natural generalizations of automorphisms of the complex surface $X$, for any automorphism clearly induces an autoequivalence of $\mathrm{D}^{\mathrm{b}}(X)$. The second motivation comes from mirror symmetry, which suggests a link between the group of autoequivalences of $\mathrm{D}^{\mathrm{b}}(X)$ and the group of diffeomorphisms or rather symplectomorphisms of the mirror dual K3 surface.

In order to study the derived category $\mathrm{D}^{\mathrm{b}}(X)$ and its autoequivalences, one needs to introduce the Mukai lattice $\widetilde{H}(X, \mathbb{Z})$ which comes with a natural weight two Hodge structure. The lattice $\widetilde{H}(X, \mathbb{Z})$ is by definition the full cohomology $H^{*}(X, \mathbb{Z})$ endowed with a modification of the intersection pairing (the Mukai pairing) obtained by introducing a sign in the pairing of $H^{0}$ with $H^{4}$. The weight two Hodge structure on $\widetilde{H}(X, \mathbb{Z})$ is by definition orthogonal with respect to the Mukai pairing and therefore determined by setting $\widetilde{H}^{2,0}(X):=H^{2,0}(X)$.

In his seminal article [29], Mukai showed that to any exact autoequivalence (of Fourier-Mukai type) $\Phi: \mathrm{D}^{\mathrm{b}}(X) \stackrel{\sim}{\rightarrow} \mathrm{D}^{\mathrm{b}}(X)$ of the bounded derived category of a projective K3 surface there is naturally associated an isomorphism

$$
\Phi^{H^{*}}: \widetilde{H}(X, \mathbb{Z}) \stackrel{\sim}{\longrightarrow} \widetilde{H}(X, \mathbb{Z})
$$

which respects the Mukai pairing and the Hodge structure, i.e. $\Phi^{H^{*}}$ is a Hodge isometry of the Mukai lattice. Thus, the natural representation $\operatorname{Aut}(X) \rightarrow \mathrm{O}\left(H^{2}(X, \mathbb{Z})\right)$ is generalized to a representation

$$
\operatorname{Aut}\left(\mathrm{D}^{\mathrm{b}}(X)\right) \longrightarrow \mathrm{O}(\widetilde{H}(X, \mathbb{Z}))
$$

The lattice $\widetilde{H}(X, \mathbb{Z})$ has signature $(4,20)$ and, in analogy to the discussion above, one says that an isometry $\rho$ of $\widetilde{H}(X, \mathbb{Z})$ is orientation preserving if under orthogonal projection a given orientation of a positive four-space in $\widetilde{H}(X, \mathbb{Z})$ coincides with the induced one on its image under $\rho$. Whether $\rho$ is orientation preserving does neither depend on the positive four-space nor on the chosen orientation of it. The main result of this paper is the proof of a conjecture that has been formulated by Szendröi in [33] as the mirror dual of Donaldson's Theorem 1.

Theorem 2. Let $\Phi: \mathrm{D}^{\mathrm{b}}(X) \stackrel{\sim}{\longrightarrow} \mathrm{D}^{\mathrm{b}}(X)$ be an exact autoequivalence of the bounded derived category of a projective $K 3$ surface $X$. Then the induced Hodge isometry $\Phi^{H^{*}}: \widetilde{H}(X, \mathbb{Z}) \stackrel{\sim}{\longrightarrow} \widetilde{H}(X, \mathbb{Z})$ is orientation preserving.

For most of the known equivalences this can be checked directly, e.g. for spherical twists and tensor products with line bundles. The case of equivalences given by the universal family of stable sheaves is more complicated and was treated in [21]. The proof of the general case, as presented in this article, is rather involved.

Theorem 2 can also be formulated for derived equivalences between two different projective K3 surfaces by using the natural orientation of the four positive directions (see Section 4.5).

Based on results of Orlov [30], it was proved in [15, 31] that any orientation preserving Hodge isometry actually occurs in the image of the representation $\operatorname{Aut}\left(\mathrm{D}^{\mathrm{b}}(X)\right) \rightarrow \mathrm{O}(\widetilde{H}(X, \mathbb{Z}))$. This can be considered as the analogue of the fact alluded to above that any orientation preserving isometry of $H^{2}(X, \mathbb{Z})$ lifts to a diffeomorphism or to the part of the Global Torelli theorem that describes the 
automorphisms of a K3 surfaces in terms of Hodge isometries of the second cohomology. Together with Theorem 1, it now allows one to describe the image of the representation $\Phi \longmapsto \Phi^{H^{*}}$ as the group of all orientation preserving Hodge isometries of the Mukai lattice $\widetilde{H}(X, \mathbb{Z})$ :

Corollary 3. For any algebraic $K 3$ surface $X$ one has

$$
\operatorname{Im}\left(\operatorname{Aut}\left(\mathrm{D}^{\mathrm{b}}(X)\right) \rightarrow \mathrm{O}(\widetilde{H}(X, \mathbb{Z}))\right)=\mathrm{O}_{+}(\widetilde{H}(X, \mathbb{Z})) .
$$

The kernel of $\operatorname{Diff}(M) \rightarrow \mathrm{O}\left(H^{2}(X, \mathbb{Z})\right)$ is largely unknown, e.g. we do not know whether it is connected. In the derived setting we have at least a beautiful conjecture due to Bridgeland which describes the kernel of the analogous representation in the derived setting as the fundamental group of an explicit period domain (see [3]).

The key idea of our approach is actually quite simple: Deforming the Fourier-Mukai kernel of a given derived equivalence yields a derived equivalence between generic K3 surfaces and those have been dealt with in [18. In particular, it is known that in the generic case the action on cohomology is orientation preserving. As the action on the lattice $\widetilde{H}(X, \mathbb{Z})$ stays constant under deformation, this proves the assertion.

What makes this program complicated and interesting, is the deformation theory that is involved. First of all, one has to make sure that the Fourier-Mukai kernel does deform sideways to any order. This can be shown if one of the two Fourier-Mukai partners is deformed along a twistor space, which itself depends on a chosen Ricci-flat metric on the K3 surface, and the other is deformed appropriately. The second problem, as usual in deformation theory, is convergence of the deformation. This point is quite delicate for at least two reasons: The Fourier-Mukai kernel is not just a coherent sheaf but a complex of coherent sheaves and the deformation we consider is not algebraic. We circumvent both problems by deforming only to the very general fibre of a formal deformation, which is a rigid analytic variety. (In fact, only the abelian and derived category of coherent sheaves on the rigid analytic variety are used and never the variety itself.) The price one pays for passing to the general fibre of the formal deformation only and not to an actual non-algebraic K3 surface is that the usual $\mathbb{C}$-linear categories are replaced by categories defined over the non-algebraically closed field $\mathbb{C}((t))$ of Laurent series.

The original paper [19] combined the results of this article and the more formal aspects now written up in [20]. We hope that splitting [19] in two shorter articles will make the structure of the discussion clearer and not lead to confusion.

The plan of this paper is as follows: In Section 2, after defining the formal setting we will work with, we show that, for a formal twistor deformation associated to a very general Kähler class, the bounded derived category of its general fibre has only one spherical object up to shift (Proposition 2.14). Hence the results of [18] can be applied. This part is based on results in [19] not covered here, which can now also be found in the separate [20]. In order to study autoequivalences of the bounded derived category of the general fibre, we construct a special stability condition for which the sections of the formal deformations yield the only stable semi-rigid objects (Proposition 2.17). As a consequence, we prove that up to shift and spherical twist any autoequivalence of the general fibre sends points to points (Proposition 2.18) and its Fourier-Mukai kernel is a sheaf (Proposition 2.19).

Section 3 deals with the deformation theory of kernels of Fourier-Mukai equivalences. In order to control the obstructions, one has to compare the Kodaira-Spencer classes of the two sides of the Fourier-Mukai equivalence, which will be done using the language of Hochschild (co)homology. In particular we show that, under suitable hypotheses on the deformation and on the Fourier-Mukai kernel, the kernel itself deforms.

In Section 4 we come back to derived equivalences of K3 surfaces and their deformations. We will prove in two steps that the first order obstruction and all the higher order obstructions are 
trivial. For one of the K3 surfaces the deformation will be given by the twistor space and for the other it will be constructed recursively. The conclusion of the proof of Theorem 2 is in Section 4.4 .

\section{The Very General tWistor Fibre of A K3 SURface}

In this section we study very special formal deformations of smooth projective K3 surfaces. The aim is to prove that the derived category of what will be called the general fibre of the formal deformation behaves similarly to the derived category of a generic non-projective K3 surface.

2.1. Formal deformations. Let $R:=\mathbb{C}[[t]]$ be the ring of power series in $t$ with field of fractions $K:=\mathbb{C}((t))$, the field of all Laurent series. For any $n$, the surjection $R \longrightarrow R_{n}:=\mathbb{C}[t] /\left(t^{n+1}\right)$ yields a closed embedding $\operatorname{Spec}\left(R_{n}\right) \subset \operatorname{Spec}(R)$, the $n$-th infinitesimal neighbourhood of $0 \in \operatorname{Spec}(R)$. The increasing sequence of closed subschemes $0=\operatorname{Spec}\left(R_{0}\right) \subset \operatorname{Spec}\left(R_{1}\right) \subset \ldots \subset \operatorname{Spec}\left(R_{n}\right) \subset \ldots$ defines the formal scheme $\operatorname{Spf}(R)$.

A formal deformation of a smooth projective variety $X$ is a smooth and proper formal $R$-scheme $\pi: \mathcal{X} \rightarrow \operatorname{Spf}(R)$, where $\mathcal{X}$ is given by an inductive system of schemes $\pi_{n}: \mathcal{X}_{n} \longrightarrow \operatorname{Spec}\left(R_{n}\right)$, smooth and proper over $R_{n}$, and isomorphisms

$$
\mathcal{X}_{n+1} \times_{R_{n+1}} \operatorname{Spec}\left(R_{n}\right) \simeq \mathcal{X}_{n}
$$

over $R_{n}$ such that $\mathcal{X}_{0}=X$. While the topological space underlying the scheme $\mathcal{X}$ is $X$, the structure sheaf of $\mathcal{X}$ is $\mathcal{O}_{\mathcal{X}}=\lim \mathcal{O}_{\mathcal{X}_{n}}$. For the rest of this paper the natural inclusions will be

denoted as follows $(m<n)$ :

$$
\begin{gathered}
\iota_{n}: \mathcal{X}_{n} \hookrightarrow \mathcal{X} \quad \text { and } \quad \iota:=\iota_{0}: X \hookrightarrow \mathcal{X} ; \\
i_{m, n}: \mathcal{X}_{m} \hookrightarrow \mathcal{X}_{n}, i_{n}:=i_{n, n+1}: \mathcal{X}_{n} \hookrightarrow \mathcal{X}_{n+1} \text {, and } j_{n}=i_{0, n}: X \hookrightarrow \mathcal{X}_{n} .
\end{gathered}
$$

Example 2.1. Examples of formal deformations of a smooth projective variety $X$ are obtained by looking at smooth and proper families $\mathbb{X} \rightarrow D$ of (usually non-algebraic) complex manifolds over a one-dimensional disk $D$ with local parameter $t$ and special fibre $X=\mathbb{X}_{0}$. The infinitesimal neighbourhoods $\mathcal{X}_{n}:=\mathbb{X} \times{ }_{D} \operatorname{Spec}\left(R_{n}\right)$, considered as $R_{n}$-schemes, form an inductive system and thus give rise to a formal $R$-scheme $\pi: \mathcal{X} \rightarrow \operatorname{Spf}(R)$. Thus, although the nearby fibres $\mathbb{X}_{t}$ of $X=\mathbb{X}_{0}$ could be non-algebraic, the construction leads to the algebraic object $\mathcal{X}$.

If $X$ is a K3 surface, examples of such families are provided by the twistor space $\pi: \mathbb{X}(\omega) \rightarrow \mathbb{P}(\omega)$ associated to a Kähler class $\omega$ on $X$. The total space $\mathbb{X}(\omega)$ is a compact complex threefold, which is never algebraic nor Kähler (see [13, Rem. 25.2]), and the projection $\pi$ is smooth and holomorphic onto the base $\mathbb{P}(\omega)$, which is non-canonically isomorphic to $\mathbb{P}^{1}$. The fibres are the complex manifolds obtained by hyperkähler rotating the original complex structure defining $X$ in the direction of the hyperkähler metric determined by $\omega$. In particular, there is a distinguished point $0 \in \mathbb{P}(\omega)$ such that the fibre $\mathbb{X}(\omega)_{0}:=\pi^{-1}(0)$ is our original K3 surface $X$. By construction, the image of the composition

$$
T_{0} \mathbb{P}(\omega) \longrightarrow H^{1}\left(X, \mathcal{T}_{X}\right) \longrightarrow H^{1}\left(X, \Omega_{X}^{1}\right)
$$

of the Kodaira-Spencer map and the contraction $v \longmapsto v\lrcorner \sigma=\sigma(v,-)$, where $\sigma \in H^{0}\left(X, \Omega_{X}^{2}\right)$ is any non-trivial holomorphic two-form, is spanned by the Kähler class $\omega$ (for further details, see [1]).

Choosing a local parameter $t$ around 0 , one gets a formal deformation $\pi: \mathcal{X} \rightarrow \operatorname{Spf}(R)$ which we call the formal twistor space of $X$. Notice that the construction depends on the choice of the Kähler class $\omega$ and of the local parameter $t$.

The $R$-linear category $\operatorname{Coh}(\mathcal{X})$ of coherent sheaves on $\mathcal{X}$ contains the full abelian subcategory $\operatorname{Coh}(\mathcal{X})_{0} \subset \operatorname{Coh}(\mathcal{X})$ consisting of all sheaves $E \in \operatorname{Coh}(\mathcal{X})$ such that $t^{n} E=0$ for $n \gg 0$. (For the definition of coherent sheaves on noetherian formal schemes see [14, Ch. II.9] or [23.) By definition $\operatorname{Coh}(\mathcal{X})_{0}$ is a Serre subcategory and the quotient category

$$
\operatorname{Coh}\left(\mathcal{X}_{K}\right):=\operatorname{Coh}(\mathcal{X}) / \operatorname{Coh}(\mathcal{X})_{0}
$$


is called the category of coherent sheaves on the general fibre. By abuse of notation, we sometimes denote $\operatorname{Coh}\left(\mathcal{X}_{K}\right)$ by $\mathcal{X}_{K}$. When $\mathcal{X}$ is a formal twistor space, we call the general fibre $\mathcal{X}_{K}$ the general twistor fibre.

For $E \in \operatorname{Coh}(\mathcal{X})$, denote by $E_{K}$ its projection in $\operatorname{Coh}\left(\mathcal{X}_{K}\right)$. The category $\operatorname{Coh}\left(\mathcal{X}_{K}\right)$ is a $K$-linear abelian category and

$$
\operatorname{Hom}_{\mathcal{X}_{K}}\left(E_{K}, F_{K}\right) \simeq \operatorname{Hom}_{\mathcal{X}}(E, F) \otimes_{R} K
$$

for any $E, F \in \mathbf{C o h}(\mathcal{X})$ (see [19, Prop. 2.4] or [20, Prop. 2.3]).

A coherent sheaf $E \in \mathbf{C o h}(\mathcal{X})$ is $R$-flat if the multiplication with $t$ yields an injective homomorphism $t: E \rightarrow E$. We denote by $\operatorname{Coh}(\mathcal{X})_{\mathrm{f}} \subset \mathbf{C o h}(\mathcal{X})$ the full additive subcategory of all $R$-flat sheaves. Observe that $\operatorname{Coh}(\mathcal{X})_{\mathrm{f}} \rightarrow \operatorname{Coh}\left(\mathcal{X}_{K}\right)$ is essentially surjective, i.e. every object $F \in \operatorname{Coh}\left(\mathcal{X}_{K}\right)$ can be lifted to an $R$-flat sheaf on $\mathcal{X}$. Indeed, if $F=E_{K}$, then $\left(E_{\mathrm{f}}\right)_{K} \simeq E_{K}=F$, where $E_{\mathrm{f}}=E / T$ and $T:=\cup \operatorname{Ker}\left(t^{n}: E \rightarrow E\right.$ ) (notice that since locally a coherent sheaf $E$ is the completion of a finitely generated module over a noetherian ring, the union stabilizes). By definition $E_{\mathrm{f}}$ is an $R$-flat lift of $F$.

Passing to derived categories, consider the full thick triangulated subcategory

$$
\mathrm{D}_{0}^{\mathrm{b}}(\mathcal{X}) \subseteq \mathrm{D}^{\mathrm{b}}(\mathcal{X}):=\mathrm{D}_{\text {coh }}^{\mathrm{b}}\left(\mathcal{O}_{\mathcal{X}} \text {-Mod }\right)
$$

consisting of complexes of $\mathcal{O}_{\mathcal{X}}$-modules with cohomologies in $\operatorname{Coh}(\mathcal{X})_{0}$. The Verdier quotient category $\mathrm{D}^{\mathrm{b}}\left(\mathcal{X}_{K}\right):=\mathrm{D}^{\mathrm{b}}(\mathcal{X}) / \mathrm{D}_{0}^{\mathrm{b}}(\mathcal{X})$ is called the derived category of the general fibre. As before, we denote by $E_{K}$ the projection to $\mathrm{D}^{\mathrm{b}}\left(\mathcal{X}_{K}\right)$ of any $E \in \mathrm{D}^{\mathrm{b}}(\mathcal{X})$. The category $\mathrm{D}^{\mathrm{b}}\left(\mathcal{X}_{K}\right)$ is a $K$-linear triangulated category and

$$
\operatorname{Hom}_{\mathcal{X}_{K}}\left(E_{K}, F_{K}\right) \simeq \operatorname{Hom}_{\mathcal{X}}(E, F) \otimes_{R} K
$$

for any $E, F \in \mathrm{D}^{\mathrm{b}}(\mathcal{X})$ (see [19, Prop. 3.9] or [20, Prop. 2.9]). In particular $\mathrm{D}^{\mathrm{b}}\left(\mathcal{X}_{K}\right)$ has finite dimensional Hom-spaces over $K$. Moreover $\operatorname{Coh}\left(\mathcal{X}_{K}\right)$ is the heart of a bounded $t$-structure in $\mathrm{D}^{\mathrm{b}}\left(\mathcal{X}_{K}\right)$.

When $X$ is a K3 surface, the main properties of $\mathrm{D}^{\mathrm{b}}\left(\mathcal{X}_{K}\right)$ are summarized by the following result which is proved in [19, Sect. 3] or [20, Thm. 1.1].

Theorem 2.2. Let $\pi: \mathcal{X} \rightarrow \operatorname{Spf}(R)$ be a formal deformation of a K3 surface $X=\mathcal{X}_{0}$. Then the derived category $\mathrm{D}^{\mathrm{b}}\left(\mathcal{X}_{K}\right)$ of its general fibre is equivalent to $\mathrm{D}^{\mathrm{b}}\left(\mathbf{C o h}\left(\mathcal{X}_{K}\right)\right)$ which is a $K$-linear K3 category.

Recall that a $K 3$ category is a triangulated category with finite dimensional Hom-spaces and such that the double shift defines a Serre functor (see [18]).

The main derived functors (tensor product, pull-back push-forward, Hom's) are well-defined at the level of derived categories of formal schemes over $\operatorname{Spf}(R)$. Moreover, all the basic properties of them (e.g. commutativity, flat base change, projection formula) hold in the formal context. All those functors are $R$-linear and hence they factorize through the derived category of the general fibre, verifying the same compatibilities (see [19, App. A.1] or [20, Sect. 2.3]). To simplify the notation, sometimes we will denote a functor and its derived version in the same manner. In the case of an immersion of (formal) schemes $j: Y \hookrightarrow Z$ and a sheaf $F \in \operatorname{Coh}(Z)$, we set $\left.F\right|_{Y}:=\mathcal{H}^{0}\left(L j^{*} F\right)$.

Remark 2.3. Let $X$ and $Y$ be smooth and projective varieties. Let $\mathcal{X}_{n}, \mathcal{Y}_{n} \rightarrow \operatorname{Spec}\left(R_{n}\right)$ be an inductive system of smooth and proper schemes such that $\mathcal{X}_{n} \times_{R_{n}} \operatorname{Spec}\left(R_{0}\right) \simeq X$ and $\mathcal{Y}_{n} \times_{R_{n}}$ $\operatorname{Spec}\left(R_{0}\right) \simeq Y$, with $n \in \mathbb{N}$. These collections yield formal deformations $\mathcal{X}, \mathcal{Y} \rightarrow \operatorname{Spf}(R)$ of $X$ and $Y$ respectively. 
i) Any bounded complex with coherent cohomology on a smooth formal scheme is perfect, i.e. locally quasi-isomorphic to a finite complex of locally free sheaves of finite type. This is however not true for $\mathcal{X}_{n}, n>0$, in which case we will have sometimes to work with

$$
\mathrm{D}_{\text {perf }}\left(\mathcal{X}_{n}\right) \subset \mathrm{D}^{\mathrm{b}}\left(\mathcal{X}_{n}\right)
$$

the full triangulated subcategory of perfect complexes on $\mathcal{X}_{n}$. For $E \in \mathrm{D}^{\mathrm{b}}(\mathcal{X})$, we set $E_{n}:=$ $L \iota_{n}^{*} E \in \mathrm{D}_{\text {perf }}\left(\mathcal{X}_{n}\right)$.

ii) Given $\mathcal{E} \in \mathrm{D}^{\mathrm{b}}\left(\mathcal{X} \times{ }_{R} \mathcal{Y}\right)$, we can define the Fourier-Mukai transform

$$
\Phi_{\mathcal{E}}: \mathrm{D}^{\mathrm{b}}(\mathcal{X}) \longrightarrow \mathrm{D}^{\mathrm{b}}(\mathcal{Y}), \quad E \longmapsto R p_{*}\left(q^{*} E \otimes^{L} \mathcal{E}\right),
$$

where $p: \mathcal{X} \times \mathcal{Y} \longrightarrow \mathcal{Y}$ and $q: \mathcal{X} \times \mathcal{Y} \rightarrow \mathcal{X}$ are the projections. All the basic properties of FourierMukai transforms valid for smooth projective varieties extend to the formal setting (see [19, App. A.2] or [20, Sect. 2.3]). Everything said also works for $\mathcal{X}_{n}$ and $\mathcal{Y}_{n}$ with the only difference that we have to assume now that the Fourier-Mukai kernel $\mathcal{E}_{n} \in \mathrm{D}^{\mathrm{b}}\left(\mathcal{X}_{n} \times_{R_{n}} \mathcal{Y}_{n}\right)$ is perfect. Analogously, one can define the Fourier-Mukai transform

$$
\Phi_{\mathcal{F}}: \mathrm{D}^{\mathrm{b}}\left(\mathcal{X}_{K}\right) \longrightarrow \mathrm{D}^{\mathrm{b}}\left(\mathcal{Y}_{K}\right)
$$

associated to an object $\mathcal{F} \in \mathrm{D}^{\mathrm{b}}\left(\left(\mathcal{X} \times_{R} \mathcal{Y}\right)_{K}\right)$. Indeed, given $\mathcal{E} \in \mathrm{D}^{\mathrm{b}}\left(\mathcal{X} \times_{R} \mathcal{Y}\right)$ with $\mathcal{E}_{K} \simeq \mathcal{F}$, by $R$-linearity, the Fourier-Mukai transform $\Phi_{\mathcal{E}}: \mathrm{D}^{\mathrm{b}}(\mathcal{X}) \rightarrow \mathrm{D}^{\mathrm{b}}(\mathcal{Y})$ descends to a Fourier-Mukai transform $\Phi_{\mathcal{F}}: \mathrm{D}^{\mathrm{b}}\left(\mathcal{X}_{K}\right) \longrightarrow \mathrm{D}^{\mathrm{b}}\left(\mathcal{Y}_{K}\right)$, i.e. one has a commutative diagram

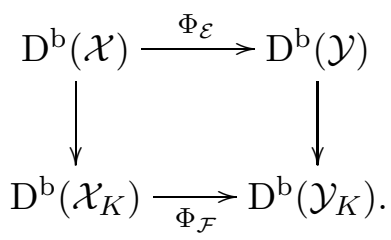

iii) Let $\mathcal{E}_{n} \in \mathrm{D}_{\text {perf }}\left(\mathcal{X}_{n} \times_{R_{n}} \mathcal{Y}_{n}\right)$, with $n \in \mathbb{N}$, be such that its restriction $\mathcal{E}_{0}:=L j_{n}^{*} \mathcal{E}_{n} \in \mathrm{D}^{\mathrm{b}}(X \times Y)$ is the kernel of a Fourier-Mukai equivalence $\Phi_{\mathcal{E}_{0}}: \mathrm{D}^{\mathrm{b}}(X) \stackrel{\sim}{\longrightarrow} \mathrm{D}^{\mathrm{b}}(Y)$. Then the Fourier-Mukai transforms $\Phi_{\mathcal{E}_{n}}: \mathrm{D}_{\text {perf }}\left(\mathcal{X}_{n}\right) \rightarrow \mathrm{D}_{\text {perf }}\left(\mathcal{Y}_{n}\right)$ and $\Phi_{\mathcal{E}_{n}}: \mathrm{D}^{\mathrm{b}}\left(\mathcal{X}_{n}\right) \rightarrow \mathrm{D}^{\mathrm{b}}\left(\mathcal{Y}_{n}\right)$ are equivalences. The same holds true for $\mathcal{E} \in \mathrm{D}^{\mathrm{b}}\left(\mathcal{X} \times_{R} \mathcal{Y}\right)$ (see [19, Prop. 3.19] or [20, Prop. 2.12]).

iv) As a consequence of iii), for $\mathcal{E} \in \mathrm{D}^{\mathrm{b}}\left(\mathcal{X} \times_{R} \mathcal{Y}\right)$ such that $\Phi_{\mathcal{E}_{0}}: \mathrm{D}^{\mathrm{b}}(X) \stackrel{\sim}{\longrightarrow} \mathrm{D}^{\mathrm{b}}(Y)$ is an equivalence, the Fourier-Mukai transform $\Phi_{\mathcal{E}_{K}}: \mathrm{D}^{\mathrm{b}}\left(\mathcal{X}_{K}\right) \stackrel{\sim}{\longrightarrow} \mathrm{D}^{\mathrm{b}}\left(\mathcal{Y}_{K}\right)$ is an equivalence, where $\mathcal{E}_{K} \in \mathrm{D}^{\mathrm{b}}\left(\left(\mathcal{X} \times_{R} \mathcal{Y}\right)_{K}\right)$ (see [19, Cor. 3.20] or [20, Cor. 2.13]).

Sheaves and complexes of sheaves are usually denoted by $E, F$, etc. The use of $\mathcal{E}, \mathcal{F}$ wants to indicate that they are Fourier-Mukai kernels, which we wish to distinguish from the objects on the source and target variety of the associated Fourier-Mukai transform.

Given a formal deformation $\pi: \mathcal{X} \rightarrow \operatorname{Spf}(R)$ of a smooth projective variety $X$, the category $\mathrm{D}^{\mathrm{b}}\left(\mathcal{X}_{K}\right)$ contains the special object $\mathcal{O}_{\mathcal{X}_{K}}:=\left(\mathcal{O}_{\mathcal{X}}\right)_{K}$. Other objects of interest for this paper are obtained as follows. A multisection is an integral formal subscheme $\mathcal{Z} \subset \mathcal{X}$ which is flat of relative dimension zero over $\operatorname{Spf}(R)$. The structure sheaf $\mathcal{O}_{\mathcal{Z}}$ of such a multisection induces an object in $\operatorname{Coh}\left(\mathcal{X}_{K}\right)$. Objects of this form will usually be denoted by $K(\mathbf{x}) \in \mathbf{C o h}\left(\mathcal{X}_{K}\right)$ and should be thought of as (structure sheaves of) closed points $\mathbf{x} \in \mathcal{X}_{K}$ of the general fibre $\mathcal{X}_{K}$. By specialization, any point $K(\mathbf{x}) \in \operatorname{Coh}\left(\mathcal{X}_{K}\right)$ determines a closed point $x \in X$ of the special fibre. The point $\mathbf{x}$ is called $K$-rational if $\mathcal{Z} \subset \mathcal{X}$ is a section, i.e. $\left.\pi\right|_{\mathcal{Z}}: \mathcal{Z} \rightarrow \operatorname{Spf}(R)$ is an isomorphism. Clearly, a closed point $\mathbf{x} \in \mathcal{X}_{K}$ is $K$-rational if and only if the natural homomorphism $K \rightarrow \operatorname{End}_{\mathcal{X}_{K}}(K(\mathbf{x}))$ is an isomorphism. 
Remark 2.4. Let $\mathcal{X}$ be a formal deformation of a K3 surface $X$ and take $F \in \mathrm{D}^{\mathrm{b}}\left(\mathcal{X}_{K}\right)$.

i) We call $F$ rigid if $\operatorname{Ext}_{\mathcal{X}_{K}}^{1}(F, F)=0$. We call $F$ spherical if it is rigid and $\operatorname{Ext}_{\mathcal{X}_{K}}^{i}(F, F) \simeq K$ if $i=0,2$. An example of spherical object is provided by $\mathcal{O}_{\mathcal{X}_{K}}$. For this use (2.3) and (2.2).

ii) The object $F$ is semi-rigid if $\operatorname{Ext}_{\mathcal{X}_{K}}^{1}(F, F)=K^{\oplus 2}$. Applying again (2.2) and Serre duality one shows that a $K$-rational point $\mathbf{x}$ is semi-rigid.

2.2. Torsion (free) sheaves on the general fibre. For a formal deformation $\mathcal{X}$ of a smooth projective variety $X$, we say that $F \in \mathbf{C o h}\left(\mathcal{X}_{K}\right)$ is torsion (resp. torsion free) if there exists a lift $E \in \operatorname{Coh}(\mathcal{X})$ of $F$ which is a torsion (resp. torsion free) sheaf on $\mathcal{X}$.

Note that $F \in \operatorname{Coh}\left(\mathcal{X}_{K}\right)$ is torsion if and only if any lift of $F$ is torsion. A torsion free $F$ always admits also lifts which are not torsion free (just add $R$-torsion sheaves). However, the lift $E$ of a torsion free $F$ is $R$-flat if and only if it is torsion free. We leave it to the reader to show that any subobject of a torsion free $F \in \mathbf{C o h}\left(\mathcal{X}_{K}\right)$ is again torsion free and that any $F \in \mathbf{C o h}\left(\mathcal{X}_{K}\right)$ admits a maximal torsion subobject $F_{\text {tor }} \subset F$ whose cokernel $F / F_{\text {tor }}$ is torsion free (use (2.1)).

Well-known arguments of Langton and Maruyama can be adapted to prove the following:

Lemma 2.5. Any torsion free $F \in \operatorname{Coh}\left(\mathcal{X}_{K}\right)$ admits an $R$-flat lift $E \in \operatorname{Coh}(\mathcal{X})$ such that the restriction $E_{0}$ of $E$ to the special fibre is a torsion free sheaf on $X$.

Proof. We shall prove the following more precise claim (cf. the proof of [16, Thm. 2.B.1]): Let $E$ be a torsion free (as $\mathcal{O}_{\mathcal{X}}$-module) coherent sheaf on $\mathcal{X}$. Then there exists a coherent subsheaf $E^{\prime} \subset E$ with $E_{0}^{\prime}:=L \iota^{*} E^{\prime}$ a torsion free sheaf and such that the inclusion induces an isomorphism $E_{K}^{\prime} \simeq E_{K}$.

Suppose there is no such $E^{\prime} \subset E$. Then we construct a strictly decreasing sequence $\ldots E^{n+1} \subset$ $E^{n} \subset \ldots \subset E^{0}=E$ inductively as follows:

$$
E^{n+1}:=\operatorname{Ker}\left(E^{n} \rightarrow E_{0}^{n} \rightarrow E_{0}^{n} /\left(E_{0}^{n}\right)_{\text {tor }}\right),
$$

where $\left(E_{0}^{n}\right)_{\text {tor }}$ means the torsion part on the special fibre. Clearly, $E_{K}^{n}=E_{K}$. For later use, we introduce $B^{n}:=\left(E_{0}^{n}\right)_{\text {tor }}$ and $G^{n}:=E_{0}^{n} / B^{n}$, which will be considered simultaneously as sheaves on the special fibre $X$ and as sheaves on $\mathcal{X}$. Then there are two exact sequences of sheaves on $\mathcal{X}$, respectively $X$

$$
0 \longrightarrow E^{n+1} \longrightarrow E^{n} \longrightarrow G^{n} \longrightarrow 0
$$

and

$$
0 \longrightarrow G^{n} \longrightarrow E_{0}^{n+1} \longrightarrow B^{n} \longrightarrow 0 .
$$

The first exact sequence is just the definition of $E^{n+1}$. For the second one we first construct the surjection $E_{0}^{n+1} \longrightarrow B^{n}$ by restricting $E^{n+1} \longrightarrow E^{n}$ to the closed fibre. The image of the resulting homomorphism $E_{0}^{n+1} \rightarrow E_{0}^{n}$ is the kernel of $E_{0}^{n} \rightarrow G^{n}$, i.e. $B^{n}$. Let $K$ be the kernel of $E_{0}^{n+1} \rightarrow B^{n}$. We will show $K \simeq G^{n}$. For this we use the two short exact sequences

$$
0 \longrightarrow t E^{n+1} \longrightarrow E^{n+1} \longrightarrow E_{0}^{n+1} \longrightarrow 0
$$

and

$$
0 \longrightarrow t E^{n} \longrightarrow E^{n+1} \longrightarrow B^{n} \longrightarrow 0
$$

where the latter one is obtained from snake lemma applied to the natural surjection $E^{n} / t E^{n}=$ $E_{0}^{n} \longrightarrow E^{n} / E^{n+1}=G^{n}$. Another diagram chase shows that $K$ sits inside the short exact sequence

$$
0 \longrightarrow t E^{n+1} \stackrel{\varphi}{\longrightarrow} t E^{n} \longrightarrow K \longrightarrow 0,
$$

where $\varphi$ is the morphism induced by the inclusion $E^{n+1} \rightarrow E^{n}$ via the isomorphisms $E_{n} \simeq t E_{n}$ and $E_{n+1} \simeq t E_{n+1}$, given by the multiplication by $t$. Thus $K \simeq G^{n}$.

As $G^{n}$ is a torsion free sheaf on $X$, one has $B^{n+1} \cap G^{n}=0$ in $E_{0}^{n+1}$. Therefore, there is a descending filtration of torsion sheaves ... $\subset B^{n+1} \subset B^{n} \subset \ldots$ and an ascending sequence of 
torsion free sheaves $\ldots \subset G^{n} \subset G^{n+1} \subset \ldots$. The support of the torsion sheaves $B^{n}$ on $X$ might have components of codimension one, but for $n \gg 0$ the filtration stabilizes in codimension one. Indeed, clearly, the support of the $B^{n}$ stabilizes for $n \gg 0$ and on there the generic rank will have to stabilize, which then means that the $B^{n}$ themselves stabilize in codimension one.

Hence, $\ldots \subset G^{n} \subset G^{n+1} \subset \ldots$ stabilizes for $n \gg 0$ in codimension one as well. In particular, the reflexive hulls do not change, i.e. $\left(G^{n}\right)^{\llcorner}=\left(G^{n+1}\right)^{\llcorner}$for $n \gg 0$. Therefore, for $n \gg 0$ the sequence $G^{n} \subset G^{n+1} \subset \ldots$ is an ascending sequence of coherent subsheaves of a fixed coherent sheaf and hence stabilizes for $n \gg 0$. This in turn implies that ... $\subset B^{n+1} \subset B^{n} \subset \ldots$ stabilizes for $n \gg 0$.

Replacing $E$ by $E^{n}$ with $n \gg 0$, we may assume that $G:=G^{0}=G^{1}=\ldots=G^{n}=\ldots$ and $0 \neq B:=B^{0}=B^{1}=\ldots=B^{n}=\ldots$. Note that this actually implies $E_{0}=G \oplus B$.

We continue with the new $E$ obtained in this way and consider the filtration $E^{n}$ for it. Now set $Q^{n}:=E / E^{n}$. Then by definition of $E^{n}$ one has $Q_{0}^{n} \simeq G$. Moreover, there exists an exact sequence

$$
0 \longrightarrow G \longrightarrow Q^{n+1} \longrightarrow Q^{n} \longrightarrow 0
$$

for $E^{n} / E^{n+1} \simeq G^{n}=G$. Next, the quotient $E \longrightarrow Q^{n}$ factorizes over $E \longrightarrow E / t^{n} E \longrightarrow Q^{n}$. Indeed, by construction $t E^{n} \subset E^{n+1}$ and thus $t^{n} E=t^{n} E^{0} \subset E^{n}$. Thus, we have a sequence of surjections $E / t^{n} E \longrightarrow Q^{n}$ of coherent sheaves on $\mathcal{X}_{n-1}$ whose restriction to the special fibre yields the surjection $E_{0} \longrightarrow G$ with non-trivial torsion kernel $B$.

One easily verifies that the system $\left(E / t^{n} E \longrightarrow Q^{n}\right)$ yields a surjection $E \longrightarrow Q$ of coherent sheaves on the formal scheme $\mathcal{X}$. Indeed, the system $\left(Q^{n}\right)$ defines a coherent sheaf on the formal scheme $\mathcal{X}$, for $G=\operatorname{Ker}\left(Q^{n+1} \rightarrow Q^{n}\right)=t^{n} Q^{n+1}$. The inclusion $t^{n} Q^{n+1} \subset G$ is obvious and $G \subset t^{n} Q^{n+1}$ can be proved inductively as follows: Suppose one has proved already that $G \subset$ $t^{k} Q^{n+1}$ for $k<n$, is the kernel of the projection $t^{k} Q^{n+1} \longrightarrow t^{k} Q^{n}$. Then use $t^{k} Q^{n+1} / t^{k+1} Q^{n+1} \simeq$ $t^{k} Q^{n} / t^{k+1} Q^{n} \simeq G$ to deduce that $G=\operatorname{Ker}\left(t^{k+1} Q^{n+1} \rightarrow t^{k+1} Q^{n}\right)$. The compatibility with the quotient maps $E / t^{n} E \longrightarrow Q^{n}$ is obvious.

Outside the support of $B$ the morphism $E \longrightarrow Q$ is an isomorphism and hence $\operatorname{Ker}(E \longrightarrow Q)$ must be torsion and non-trivial. This contradicts the assumption on $E$.

2.3. The $K$-group of the general fibre. With the usual notation, for $E, E^{\prime} \in \mathrm{D}^{\mathrm{b}}(X)$ one sets:

$$
\chi_{0}\left(E, E^{\prime}\right):=\sum(-1)^{i} \operatorname{dim}_{\mathbb{C}} \operatorname{Ext}_{X}^{i}\left(E, E^{\prime}\right)
$$

and analogously for $F, F^{\prime} \in \mathrm{D}^{\mathrm{b}}\left(\mathcal{X}_{K}\right)$ :

$$
\chi_{K}\left(F, F^{\prime}\right):=\sum(-1)^{i} \operatorname{dim}_{K} \operatorname{Ext}_{\mathcal{X}_{K}}^{i}\left(F, F^{\prime}\right) .
$$

Recall that (see [19, Cor. 3.15 and 3.16] or [20, Cor. 3.2 and 3.3]) for $E, E^{\prime} \in \mathrm{D}^{\mathrm{b}}(\mathcal{X})$, one has

$$
\chi_{0}\left(E_{0}, E_{0}^{\prime}\right)=\chi\left(E_{K}, E_{K}^{\prime}\right)
$$

and the following semi-continuity result

$$
\operatorname{dim}_{\mathbb{C}} \operatorname{Hom}_{X}\left(E_{0}, E_{0}^{\prime}\right) \geq \operatorname{dim}_{K} \operatorname{Hom}_{\mathcal{X}_{K}}\left(E_{K}, E_{K}^{\prime}\right),
$$

where $E_{0}$ and $E_{0}^{\prime}$ are the restrictions of $E$ and $E^{\prime}$ to the special fibre.

Let us now consider the $K$-groups of the various derived categories: $K(X):=K\left(\mathrm{D}^{\mathrm{b}}(X)\right)$, $K(\mathcal{X}):=K\left(\mathrm{D}^{\mathrm{b}}(\mathcal{X})\right)$, and $K\left(\mathcal{X}_{K}\right):=K\left(\mathrm{D}^{\mathrm{b}}\left(\mathcal{X}_{K}\right)\right)$. We say that a class $[E] \in K(X)$ is numerically trivial, $[E] \sim 0$, if $\chi_{0}\left(E, E^{\prime}\right)=0$ for all $E^{\prime} \in \mathrm{D}^{\mathrm{b}}(X)$. Numerical equivalence for the general fibre is defined similarly in terms of $\chi_{K}$. Set

$$
\mathcal{N}(X):=K(X) / \sim \quad \text { and } \quad \mathcal{N}\left(\mathcal{X}_{K}\right):=K\left(\mathcal{X}_{K}\right) / \sim .
$$


Lemma 2.6. Sending $[F]=\left[E_{K}\right] \in \mathcal{N}\left(\mathcal{X}_{K}\right)$ (where $E \in \mathrm{D}^{\mathrm{b}}(\mathcal{X})$ is any lift of $F$ ) to $\left[E_{0}\right] \in \mathcal{N}(X)$ determines an injective linear map

$$
\text { res }: \mathcal{N}\left(\mathcal{X}_{K}\right) \longrightarrow \mathcal{N}(X) / \iota^{*} K(\mathcal{X})^{\perp} .
$$

(The orthogonal complement is taken with respect to $\chi_{0}$. )

Proof. The linearity of the map is evident, but in order to show that it is well-defined one needs that $\chi_{K}\left(E_{K},-\right) \equiv 0$ implies $\chi_{0}\left(E_{0}, E_{0}^{\prime}\right)=0$ for all $E^{\prime} \in \mathrm{D}^{\mathrm{b}}(\mathcal{X})$. This follows from (2.2).

In order to prove injectivity of res, suppose $E_{0} \in \iota^{*} K(\mathcal{X})^{\perp}$. Then $\chi_{K}\left(E_{K}, E_{K}^{\prime}\right)=\chi_{0}\left(E_{0}, E_{0}^{\prime}\right)=0$ for all $\left[E^{\prime}\right] \in K(\mathcal{X})$. Since $K(\mathcal{X}) \rightarrow K\left(\mathcal{X}_{K}\right)$ is surjective, this proves the claim.

Remark 2.7. In fact, res can be lifted to a map

$$
K\left(\mathcal{X}_{K}\right) \longrightarrow K(X)
$$

which will be used only once (see the proof of Corollary 4.9). To show that the natural map $\left[E_{K}\right] \longmapsto\left[E_{0}\right]$ is well-defined, it suffices to show that any $R$-torsion sheaf $E \in \operatorname{Coh}(\mathcal{X})$ leads to a trivial class $\left[L \iota^{*} E\right]=\left[E_{0}\right]$ in $K(X)$.

As any $R$-torsion sheaf admits a filtration with quotients living on $\mathcal{X}_{0}=X$, it is enough to prove that $0=\left[L \iota^{*} \iota_{*} G\right] \in K(X)$ for any $G \in \mathbf{C o h}(X)$. For this, we complete the adjunction morphism $L \iota^{*} \iota_{*} G \longrightarrow G$ to the distinguished triangle

$$
G[1] \longrightarrow L \iota^{*} \iota_{*} G \longrightarrow G
$$

which shows $\left[L \iota^{*} \iota_{*} G\right]=[G]+[G[1]]=0$. For the existence of (2.4) see e.g. [17, Cor. 11.4]. The proof there can be adapted to the formal setting.

2.4. The general fibre of a very general twistor space. Let $\pi: \mathcal{X} \rightarrow \operatorname{Spf}(R)$ be a formal twistor space of a K3 surface $X$ associated to a Kähler class $\omega$. In the following, $\omega$ has to be chosen very general in order to ensure that only the trivial line bundle $\mathcal{O}_{X}$ deforms sideways. Here is the precise definition we shall work with.

Definition 2.8. A Kähler class $\omega \in H^{1,1}(X, \mathbb{R})$ is called very general if there is no non-trivial integral class $0 \neq \alpha \in H^{1,1}(X, \mathbb{Z})$ orthogonal to $\omega$, i.e. $\omega^{\perp} \cap H^{1,1}(X, \mathbb{Z})=0$.

The twistor space associated to a very general Kähler class will be called a very general twistor space and its general fibre a very general twistor fibre.

Remark 2.9. Thus the set of very general Kähler classes is the complement (inside the Kähler cone $\left.\mathcal{K}_{X}\right)$ of the countable union of all hyperplanes $\alpha^{\perp} \subset H^{1,1}(X, \mathbb{R})$ with $0 \neq \alpha \in H^{1,1}(X, \mathbb{Z})$ and is, therefore, not empty. Moreover, very general Kähler classes always exist also in $\operatorname{Pic}(X) \otimes \mathbb{R}$.

In the next proposition we collect the consequences of this choice that will be used in the following discussion.

Proposition 2.10. Let $\omega$ be a very general Kähler class on a K3 surface $X$ and let $\pi: \mathcal{X} \rightarrow \operatorname{Spf}(R)$ be the induced formal twistor space. Then the following conditions hold:

i) Any line bundle on $\mathcal{X}$ is trivial.

ii) Let $\mathcal{Z} \subset \mathcal{X}$ be an $R$-flat formal subscheme. Then either the projection $\pi: \mathcal{Z} \rightarrow \operatorname{Spf}(R)$ is of relative dimension zero or $\mathcal{Z}=\mathcal{X}$.

iii) The Mukai vector $v:=\mathrm{ch} \cdot \sqrt{\operatorname{td}(X)}$ and the restriction map res (see Lemma 2.6) define isomorphisms

$$
v \circ \text { res }: \mathcal{N}\left(\mathcal{X}_{K}\right) \stackrel{\sim}{\longrightarrow} \mathcal{N}(X) / \iota^{*} K(\mathcal{X})^{\perp} \stackrel{\sim}{\longrightarrow}\left(H^{0} \oplus H^{4}\right)(X, \mathbb{Z}) \simeq \mathbb{Z}^{\oplus 2}
$$


Proof. i) As $\omega$ is very general, even to first order no integral $(1,1)$-class on $X$ stays pure. Thus, in fact any line bundle on $\mathcal{X}_{1}$ is trivial (see e.g. [13, Lemma 26.4]).

ii) The second assertion holds without any genericity assumption on the Kähler class $\omega$ and goes back to Fujiki [12. The case of relative dimension one can also be excluded using i).

iii) For the second isomorphism we need to show that $\iota^{*} K(\mathcal{X})^{\perp} / \sim=H^{1,1}(X, \mathbb{Z})$. As has been used already in the proof of $\mathrm{i})$, no class in $H^{1,1}(X, \mathbb{Z})$ deforms even to first order. In other words, the image of $\iota^{*} K(\mathcal{X})$ in $\mathcal{N}(X)$ is contained in $H^{0}(X, \mathbb{Z}) \oplus H^{4}(X, \mathbb{Z})$ and thus orthogonal to $H^{2}(X)$ and in particular to $H^{1,1}(X, \mathbb{Z})$, proving $H^{1,1}(X, \mathbb{Z}) \subset \iota^{*} K(\mathcal{X})^{\perp}$.

To prove that the inclusion $H^{1,1}(X, \mathbb{Z}) \subset \iota^{*} K(\mathcal{X})^{\perp}$ is an equality, consider $\mathcal{O}_{\mathcal{X}}$ and the structure sheaf $\mathcal{O}_{L_{x}}$ of any section through a given closed point $x \in X$. Then $\iota^{*} \mathcal{O}_{\mathcal{X}} \simeq \mathcal{O}_{X}$ and $\iota^{*} \mathcal{O}_{L_{x}} \simeq k(x)$ with Mukai vectors $(1,0,1)$ and $(0,0,1)$, respectively. These two vectors form a basis of $\left(H^{0} \oplus H^{4}\right)(X, \mathbb{Z})$ and their images in $\mathcal{N}(X) / \iota^{*} K(\mathcal{X})^{\perp}$ are linearly independent, because $\chi_{0}\left(k(x), \iota^{*} \mathcal{O}_{L_{x}}\right)=\chi_{0}(k(x), k(x))=0$ but $\chi_{0}\left(\mathcal{O}_{X}, \iota^{*} \mathcal{O}_{L_{x}}\right) \neq 0$. This proves that the inclusion $H^{1,1}(X, \mathbb{Z}) \subset \iota^{*} K(\mathcal{X})^{\perp}$ cannot be strict. Hence we get the second isomorphism.

The injectivity of the map res has been shown in general in Lemma 2.6 and $\left[\mathcal{O}_{X}\right]$ and $[k(x)]$, spanning $\mathcal{N}(X) / \iota^{*} K(\mathcal{X})^{\perp}$, are clearly in the image of it.

Example 2.11. Under the assumptions of Proposition 2.10, we often write $(r, s)$ instead of $(r, 0, s)$ for the Mukai vector in the image of $v \circ$ res.

i) If $F$ is a non-trivial torsion free sheaf on $\mathcal{X}_{K}$, then $v(\operatorname{res}(F))=(r, s)$ with $r>0$.

ii) For any closed point $\mathbf{y} \in \mathcal{X}_{K}$ one has $v(\operatorname{res}(K(\mathbf{y})))=(0, d)$, where $d$ is the degree (over $\operatorname{Spf}(R))$ of the multisection $\mathcal{Z} \subset \mathcal{X}$ corresponding to $\mathbf{y}$.

iii) If $F \in \operatorname{Coh}\left(\mathcal{X}_{K}\right)$ with $v(\operatorname{res}(F))=(0,0)$, then $F=0$. Indeed, if $E$ is an $R$-flat lift of $F$, then $E_{0}$ would be a sheaf concentrated in dimension zero without global sections. Hence $E_{0}=0$ and then also $E=0$.

The restriction $E_{0}$ of an $R$-flat lift $E$ of a torsion $F \in \mathbf{C o h}\left(\mathcal{X}_{K}\right)$ is a torsion sheaf on the special fibre $\mathcal{X}_{0} \simeq X$ with zero-dimensional support (use Proposition 2.10). The structure of torsion sheaves on the general twistor fibre is described by the following result.

Corollary 2.12. Let $\pi: \mathcal{X} \rightarrow \operatorname{Spf}(R)$ be as in Proposition 2.10 .

i) Any torsion sheaf $F \in \operatorname{Coh}\left(\mathcal{X}_{K}\right)$ can be written as a direct sum $\bigoplus F_{i}$ such that each $F_{i}$ admits a filtration with all quotients of the form $K\left(\mathbf{y}_{i}\right)$ for some point $\mathbf{y}_{i} \in \mathcal{X}_{K}$.

ii) If $F \in \operatorname{Coh}\left(\mathcal{X}_{K}\right)$ is a non-trivial torsion free object and $0 \neq F^{\prime} \in \operatorname{Coh}\left(\mathcal{X}_{K}\right)$ is torsion, then $\operatorname{Hom}_{\mathcal{X}_{K}}\left(F, F^{\prime}\right) \neq 0$.

Proof. i) Indeed, if one lifts $F$ to an $R$-flat sheaf $E$, then $E$ is supported on a finite union of irreducible multisections $\mathcal{Z}_{i} \subset \mathcal{X}$. If only one $\mathcal{Z}_{1}$ occurs, $E$ can be filtered such that all quotients are isomorphic to $\mathcal{O}_{\mathcal{Z}_{1}}$ which induces the claimed filtration of $F=E_{K}$. Thus, it suffices to show that for two distinct multisections $\mathcal{Z}_{1}, \mathcal{Z}_{2} \subset \mathcal{X}$ inducing points $\mathbf{y}_{1} \neq \mathbf{y}_{2} \in \mathcal{X}_{K}$ in the general fibre there are no non-trivial extensions, i.e. $\operatorname{Ext}_{\mathcal{X}_{K}}^{1}\left(K\left(\mathbf{y}_{1}\right), K\left(\mathbf{y}_{2}\right)\right)=0$. If $\mathcal{Z}_{1}$ and $\mathcal{Z}_{2}$ specialize to distinct points $y_{1} \neq y_{2} \in X$ (with multiplicities), then this obvious by semi-continuity (2.3). For $y_{1}=y_{2}$ one still has $\chi_{K}\left(K\left(\mathbf{y}_{1}\right), K\left(\mathbf{y}_{2}\right)\right)=\chi_{0}\left(k\left(y_{1}\right), k\left(y_{2}\right)\right)=0$ due to (2.2). Using Serre duality, it therefore suffices to show that $\operatorname{Hom}_{\mathcal{X}_{K}}\left(K\left(\mathbf{y}_{1}\right), K\left(\mathbf{y}_{2}\right)\right)=0$. If $f: \mathcal{O}_{\mathcal{Z}_{1}} \longrightarrow \mathcal{O}_{\mathcal{Z}_{2}}$ is a non-trivial homomorphism, then its image would be the structure sheaf of a subscheme of $\mathcal{X}$ contained in $\mathcal{Z}_{1}$ and in $\mathcal{Z}_{2}$. Clearly, the irreducible multisections $\mathcal{Z}_{i}$ do not contain any proper subschemes.

ii) By i) it suffices to show that $\operatorname{Hom}_{\mathcal{X}_{K}}(F, K(\mathbf{y})) \neq 0$ for any closed point $\mathbf{y} \in \mathcal{X}_{K}$ and any torsion free $F \in \operatorname{Coh}\left(\mathcal{X}_{K}\right)$. Using Serre duality, one knows $\operatorname{Ext}_{\mathcal{X}_{K}}^{2}(F, K(\mathbf{y})) \simeq \operatorname{Hom}_{\mathcal{X}_{K}}(K(\mathbf{y}), F)^{*}=0$. Thus, $\chi_{K}(F, K(\mathbf{y}))=r \cdot d>0$, where $r$ is given by $v(\operatorname{res}(F))=(r, s)$ and $d$ is the degree of the multisection corresponding to $\mathbf{y} \in \mathcal{X}_{K}$, implies the assertion.

Clearly, in the decomposition $F \simeq \bigoplus F_{i}$ we may assume that the points $\mathbf{y}_{i}$ are pairwise distinct, which we will usually do. 
Remark 2.13. Later we shall use Proposition 2.10 and Corollary 2.12 under slightly weaker assumptions. One easily checks that it suffices to assume that the first order neighbourhood of $\mathcal{X} \rightarrow \operatorname{Spf}(R)$ is induced by a generic twistor space. In fact, the only assumption that is really needed is that $\mathcal{O}_{\mathcal{X}}$ is the only line bundle on $\mathcal{X}$.

2.5. Spherical objects on the very general twistor fibre. The proof of the following proposition is almost a word by word copy of the proof of [18, Lemma 4.1] and is included only to show that indeed the techniques well-known for classical K3 surfaces work as well for the general twistor fibre.

Proposition 2.14. Suppose $\pi: \mathcal{X} \rightarrow \operatorname{Spf}(R)$ is the formal twistor space of a K3 surface $X$ associated to a very general Kähler class. Then

i) The structure sheaf $\mathcal{O}_{\mathcal{X}_{K}}$ is the only indecomposable rigid object in $\operatorname{Coh}\left(\mathcal{X}_{K}\right)$.

ii) Up to shift, $\mathcal{O}_{\mathcal{X}_{K}}$ is the only quasi-spherical object in $\mathrm{D}^{\mathrm{b}}\left(\mathcal{X}_{K}\right)$ (see [18, Def. 2.5]).

iii) The K3 category $\mathrm{D}^{\mathrm{b}}\left(\mathcal{X}_{K}\right)$ satisfies condition $(*)$ in [18, Rem. 2.8].

Proof. Proposition 2.14 in [18] shows that i) implies ii) and iii). Thus, only i) needs a proof.

First, let us show that any rigid $F \in \mathbf{C o h}\left(\mathcal{X}_{K}\right)$ is torsion free. If not, the standard exact sequence $0 \longrightarrow F_{\text {tor }} \rightarrow F \longrightarrow F^{\prime} \longrightarrow 0$ (see Section [2.2) together with $\operatorname{Hom}_{\mathcal{X}}\left(F_{\text {tor }}, F^{\prime}\right)=0$ and [18, Lemma 2.7] would show that also $F_{\text {tor }}$ is rigid. However, due to Corollary [2.12, i) $\left[F_{\text {tor }}\right] \in K\left(\mathcal{X}_{K}\right)$ equals a direct sum of sheaves of the form $K(\mathbf{y})$. As $\chi_{K}\left(K\left(\mathbf{y}_{1}\right), K\left(\mathbf{y}_{2}\right)\right)=0$ for arbitrary points $\mathbf{y}_{1}, \mathbf{y}_{2} \in \mathcal{X}_{K}$, one also has $\chi_{K}\left(F_{\text {tor }}, F_{\text {tor }}\right)=0$, which obviously contradicts rigidity of a non-trivial $F_{\text {tor }} \in \operatorname{Coh}\left(\mathcal{X}_{K}\right)$.

As an illustration of the techniques, let us next prove that $\mathcal{O}_{\mathcal{X}_{K}}$ is the only spherical object in $\mathrm{D}^{\mathrm{b}}\left(\mathcal{X}_{K}\right)$ that is contained in $\operatorname{Coh}\left(\mathcal{X}_{K}\right)$. Suppose $F \in \operatorname{Coh}\left(\mathcal{X}_{K}\right)$ is spherical and let $E \in \operatorname{Coh}(\mathcal{X})$ be an $R$-flat torsion free lift of $F$. Then, by (2.2), one has $2=\chi_{K}(F, F)=\chi_{0}\left(E_{0}, E_{0}\right)$, i.e. $v\left(E_{0}\right)=v(\operatorname{res}(F))= \pm(1,0,1)$. As $F$ (and hence $E_{0}$ ) is a sheaf, we must have $v\left(E_{0}\right)=(1,0,1)$. In other words, $F$ and $\mathcal{O}_{\mathcal{X}_{K}}$ are numerically equivalent and, in particular, $\chi_{K}\left(\mathcal{O}_{\mathcal{X}_{K}}, F\right)=2$. The latter implies the existence of a non-trivial $f: F \rightarrow \mathcal{O}_{\mathcal{X}_{K}}$ or a non-trivial $f: \mathcal{O}_{\mathcal{X}_{K}} \longrightarrow F$. Now we conclude by observing that any non-trivial $f: G_{1} \rightarrow G_{2}$ in $\operatorname{Coh}\left(\mathcal{X}_{K}\right)$ between torsion free $G_{1}$ and $G_{2}$ with $v\left(\operatorname{res}\left(G_{1}\right)\right)=v\left(\operatorname{res}\left(G_{2}\right)\right)=(1,0,1)$ is necessarily an isomorphism. Indeed, kernel and image of such an $f$ are either trivial or torsion free of rank one. Since the rank is additive and $f \neq 0$, in fact $f$ is injective. The cokernel of the injective $f: G_{1} \hookrightarrow G_{2}$ would be an $H \in \mathbf{C o h}\left(\mathcal{X}_{K}\right)$ with trivial Mukai vector $v(\operatorname{res}(H))=0$ and hence $H=0$ (see Example 2.11, iii)), i.e. $f$ is an isomorphism.

Consider now an arbitrary rigid indecomposable $F \in \mathbf{C o h}\left(\mathcal{X}_{K}\right)$ and let $\left(r_{0}, s_{0}\right)=v(\operatorname{res}(F))$. Then $\chi_{K}(F, F)=2 r_{0} s_{0}>0$ and hence $s_{0}>0$. Therefore, $\chi_{K}\left(\mathcal{O}_{\mathcal{X}_{K}}, F\right)=r_{0}+s_{0}>0$. Suppose $\operatorname{Hom}_{\mathcal{X}_{K}}\left(\mathcal{O}_{\mathcal{X}_{K}}, F\right) \neq 0$ and consider a short exact sequence of the form

$$
0 \longrightarrow \mathcal{O}_{\mathcal{X}_{K}}^{\oplus r} \longrightarrow F \stackrel{\xi}{\longrightarrow} F^{\prime} \longrightarrow 0 .
$$

We claim that then $F^{\prime}$ must be torsion free. If not, the extension

$$
0 \longrightarrow \mathcal{O}_{\mathcal{X}_{K}}^{\oplus r} \longrightarrow \xi^{-1}\left(F_{\text {tor }}^{\prime}\right) \longrightarrow F_{\text {tor }}^{\prime} \longrightarrow 0
$$

would necessarily be non-trivial, for $\xi^{-1}\left(F_{\text {tor }}^{\prime}\right) \subset F$ is torsion free. On the other hand, by Serre duality and Corollary 2.12, i), one has $\operatorname{Ext}_{\mathcal{X}_{K}}^{1}\left(F_{\text {tor }}^{\prime}, \mathcal{O}_{\mathcal{X}_{K}}\right)=0$. Indeed, $\operatorname{Ext}_{\mathcal{X}_{K}}^{1}\left(K(\mathbf{y}), \mathcal{O}_{\mathcal{X}_{K}}\right) \simeq$ $\operatorname{Ext}_{\mathcal{X}_{K}}^{1}\left(\mathcal{O}_{\mathcal{X}_{K}}, K(\mathbf{y})\right)^{*} \simeq\left(R^{1} \pi_{*} \mathcal{O}_{\mathcal{Z}} \otimes K\right)^{*}=0$, where $\mathcal{Z} \subset \mathcal{X}$ is the multisection corresponding to $\mathbf{y} \in \mathcal{X}_{K}$.

Now choose $r$ maximal in (2.5). As any $0 \neq s \in \operatorname{Hom}_{\mathcal{X}_{K}}\left(\mathcal{O}_{\mathcal{X}_{K}}, F^{\prime}\right)$ defines an injection (use $F^{\prime}$ torsion free), the lift of $s$ to $\tilde{s} \in \operatorname{Hom}_{\mathcal{X}_{K}}\left(\mathcal{O}_{\mathcal{X}_{K}}, F\right)$, which exists as $\mathcal{O}_{\mathcal{X}_{K}}$ is spherical, together with the given inclusion $\mathcal{O}_{\mathcal{X}_{K}}^{\oplus r} \subset F$ would yield an inclusion $\mathcal{O}_{\mathcal{X}_{K}}^{\oplus r+1} \subset F$ contradicting the maximality of 
$r$. Thus, for maximal $r$ the cokernel $F^{\prime}$ satisfies $\operatorname{Hom}_{\mathcal{X}_{K}}\left(\mathcal{O}_{\mathcal{X}_{K}}, F^{\prime}\right)=0$ and by [18, Lemma 2.7] $F^{\prime}$ would be rigid as well. Then by induction on the rank, we may assume $F^{\prime} \simeq \mathcal{O}_{\mathcal{X}_{K}}^{\oplus s^{\prime}}$ and, since $\mathcal{O}_{\mathcal{X}_{K}}$ is spherical, this contradicts the assumption that $F$ is indecomposable.

Eventually, one has to deal with the case that $\operatorname{Hom}_{\mathcal{X}_{K}}\left(\mathcal{O}_{\mathcal{X}_{K}}, F\right)=0$, but $\operatorname{Hom}_{\mathcal{X}_{K}}\left(F, \mathcal{O}_{\mathcal{X}_{K}}\right) \neq 0$. To this end, consider the reflexive hull $F^{\vee}$. By definition $F^{\checkmark \checkmark}=\left(E^{\vee}\right)_{K}$, where $E$ is an $R$-flat lift of $F$. As we have seen above, $F$ and hence $E$ is torsion free. Thus, $F \rightarrow F^{\smile}$ is injective. The quotient map $F^{\curlyvee} \longrightarrow\left(F^{\checkmark} / F\right)$ deforms non trivially if $\left(F^{\smile \checkmark} / F\right) \neq 0$, e.g. by deforming the support of the quotient (use Corollary 2.12, ii)). This would contradict the rigidity of $F$. Hence $F \simeq F^{\smile}$. Then $\operatorname{Hom}_{\mathcal{X}_{K}}\left(F, \mathcal{O}_{\mathcal{X}_{K}}\right)=\operatorname{Hom}_{\mathcal{X}_{K}}\left(\mathcal{O}_{\mathcal{X}_{K}}, F^{\vee}\right)$ and we can apply the previous discussion to the rigid sheaf $F^{`}$.

Let us now consider the spherical twist

$$
T_{K}:=T_{\mathcal{O}_{\mathcal{X}_{K}}}=\Phi_{\left(\mathcal{I}_{\Delta}\right)_{K}[1]}: \mathrm{D}^{\mathrm{b}}\left(\mathcal{X}_{K}\right) \stackrel{\sim}{\longrightarrow} \mathrm{D}^{\mathrm{b}}\left(\mathcal{X}_{K}\right)
$$

associated to the spherical object $\mathcal{O}_{\mathcal{X}_{K}} \in \mathrm{D}^{\mathrm{b}}\left(\mathcal{X}_{K}\right)$, i.e. the Fourier-Mukai equivalence with kernel $\left(\mathcal{I}_{\Delta}\right)_{K}$ [1], where $\Delta$ is the diagonal in $\mathcal{X} \times{ }_{R} \mathcal{X}$. We have the following consequence of the previous result, which will be used in the proof of Proposition 2.18.

Corollary 2.15. ([18, Proposition 2.18.) Suppose $\sigma$ is a stability condition on $\mathrm{D}^{\mathrm{b}}\left(\mathcal{X}_{K}\right)$. If $F \in \mathrm{D}^{\mathrm{b}}\left(\mathcal{X}_{K}\right)$ is semi-rigid with $\sum \operatorname{dim}_{K} \operatorname{Ext}_{\mathcal{X}_{K}}^{i}\left(\mathcal{O}_{\mathcal{X}_{K}}, F\right)=1$, then there exists an integer $n$ such that $T_{K}^{n}(F)$ is $\sigma$-stable.

For the notion of stability conditions in the sense of Bridgeland and Douglas see [3].

2.6. Stability conditions on the very general twistor fibre. The next task consists of actually constructing one explicit stability condition. Following the arguments in [18, it should be possible to classify all stability conditions on $\mathrm{D}^{\mathrm{b}}\left(\mathcal{X}_{K}\right)$ for $\mathcal{X}_{K}$ the very general twistor fibre as before. However, for our purpose this is not needed.

We shall next mimic the definition of a particular stability condition for general non-projective K3 surfaces introduced in [18, Sect. 4]. Fix a real number $u<-1$ and let $\mathcal{F}, \mathcal{T} \subset \mathbf{C o h}\left(\mathcal{X}_{K}\right)$ be the full additive subcategories of all torsion free respectively torsion sheaves $F \in \operatorname{Coh}\left(\mathcal{X}_{K}\right)$.

Lemma 2.16. The full subcategories $\mathcal{F}, \mathcal{T} \subset \operatorname{Coh}\left(\mathcal{X}_{K}\right)$ form a torsion theory for the abelian category $\operatorname{Coh}\left(\mathcal{X}_{K}\right)$.

Proof. For the definition of torsion theories see e.g. 3. Let $F \in \mathbf{C o h}\left(\mathcal{X}_{K}\right)$ and $E \in \operatorname{Coh}(\mathcal{X})$ with $E_{K} \simeq F$. Consider the short exact sequence $0 \rightarrow E_{\text {tor }} \rightarrow E \rightarrow E / E_{\text {tor }} \rightarrow 0$ of coherent sheaves on $\mathcal{X}$. Its restriction to $\mathcal{X}_{K}$, i.e. its image in $\operatorname{Coh}\left(\mathcal{X}_{K}\right)$, is still a short exact sequence, which decomposes $F$ into the torsion part $\left(E_{\text {tor }}\right)_{K}$ and its torsion free part $\left(E / E_{\text {tor }}\right)_{K}$. As there are no non-trivial homomorphisms from a torsion sheaf on $\mathcal{X}$ to a torsion free one, the same holds true in $\operatorname{Coh}\left(\mathcal{X}_{K}\right)$.

The heart of the $t$-structure associated to this torsion theory is the abelian category

$$
\mathcal{A} \subset \mathrm{D}^{\mathrm{b}}\left(\mathbf{C o h}\left(\mathcal{X}_{K}\right)\right) \simeq \mathrm{D}^{\mathrm{b}}\left(\mathcal{X}_{K}\right)
$$

consisting of all complexes $F \in \mathrm{D}^{\mathrm{b}}\left(\mathbf{C o h}\left(\mathcal{X}_{K}\right)\right)$ concentrated in degree 0 and -1 with $\mathcal{H}^{0}(F) \in$ $\operatorname{Coh}\left(\mathcal{X}_{K}\right)$ torsion and $\mathcal{H}^{-1}(F) \in \mathbf{C o h}\left(\mathcal{X}_{K}\right)$ torsion free. On this heart, one defines the additive function

$$
Z: \mathcal{A} \longrightarrow \mathbb{C}, F \longmapsto-u \cdot r-s
$$

where $(r, s)=v(\operatorname{res}(F))$. Note that by definition $Z$ takes values only in $\mathbb{R}$.

Proposition 2.17. The above construction defines a locally finite stability condition $\sigma$ on $\mathrm{D}^{\mathrm{b}}\left(\mathcal{X}_{K}\right)$. Moreover, if $F \in \mathrm{D}^{\mathrm{b}}\left(\mathcal{X}_{K}\right)$ is $\sigma$-stable and semi-rigid with $\operatorname{End}_{\mathcal{X}_{K}}(F) \simeq K$, then, up to shift, $F$ is a $K$-rational point $K(\mathbf{x})$. 
Proof. Let us first show that $Z(F) \in \mathbb{R}_{<0}$ for any non-trivial $F \in \mathcal{A}$. The Mukai vector of a torsion $F \in \operatorname{Coh}\left(\mathcal{X}_{K}\right)$ is of the form $v(\operatorname{res}(F))=(0, s)$ with $s=-c_{2}\left(E_{0}\right)$, where $E$ is an $R$-flat lift of $F$. Thus, $E_{0}$ is a non-trivial torsion sheaf on $X$ with zero-dimensional support and therefore $0<h^{0}\left(E_{0}\right)=\chi\left(E_{0}\right)=-c_{2}\left(E_{0}\right)$. Hence, $Z(F) \in \mathbb{R}_{<0}$.

Let now $F \in \operatorname{Coh}\left(\mathcal{X}_{K}\right)$ be torsion free. Then due to Lemma 2.5, there exists an $R$-flat lift $E \in \operatorname{Coh}(\mathcal{X})$ with $E_{0}$ a torsion free sheaf. We have to show that $u \cdot \operatorname{rk}\left(E_{0}\right)+s\left(E_{0}\right)<0$ or, equivalently, $\mathrm{c}_{2}\left(E_{0}\right)>\operatorname{rk}\left(E_{0}\right) \cdot(u+1)$. The inequality is linear in short exact sequences and holds for all ideal sheaves $\mathcal{I}_{Z} \subset \mathcal{O}_{X}$ of (possibly empty) zero-dimensional subschemes $Z \subset X$. By induction on the rank, we can therefore reduce to the case that $H^{0}\left(X, E_{0}\right)=0$ and $H^{2}\left(X, E_{0}\right) \simeq$ $\operatorname{Hom}_{X}\left(E_{0}, \mathcal{O}_{X}\right)^{*}=0$. (Indeed, e.g. any non trivial global section of $E_{0}$ induces an injection $\mathcal{O}_{X} \subset E_{0}$ whose cokernel has fewer global sections. Since $\mathcal{O}_{X}$ clearly satisfies the inequality, which is linear in short exact sequences, it then suffices to verify the inequality for the quotient which is of smaller rank than the original $E_{0}$. Since torsion sheaves also satisfy the inequality, one can divide out by the torsion part of the quotient to get again a torsion free sheaf.) But then $\chi\left(E_{0}\right) \leq 0$ and hence the Riemann-Roch formula yields $\mathrm{c}_{2}\left(E_{0}\right) \geq 2 \mathrm{rk}\left(E_{0}\right)>0$.

In order to verify the Harder-Narasimhan property of $\sigma$, one shows that the abelian category $\mathcal{A}$ is noetherian and artinian. At the same time, this then proves that $\sigma$ is locally finite. If $F \in \mathcal{A}$, then $\mathcal{H}^{-1}(F)[1], \mathcal{H}^{0}(F) \in \mathcal{A}$ and the distinguished triangle $0 \longrightarrow \mathcal{H}^{-1}(F)[1] \longrightarrow F \longrightarrow \mathcal{H}^{0}(F) \longrightarrow 0$ is thus an exact sequence in $\mathcal{A}$. So, if $F \supset F_{1} \supset F_{2} \supset \ldots$ is a descending sequence in $\mathcal{A}$, then the $\mathcal{H}^{-1}$ of it form a descending sequence of torsion free sheaves. Due to rank considerations this eventually stabilizes (the quotients $\mathcal{H}^{-1}\left(F_{i}\right) / \mathcal{H}^{-1}\left(F_{i+1}\right)$ are also torsion free!) and from then on one has a decreasing sequence of torsion sheaves $\mathcal{H}^{0}\left(F_{i}\right) \supset \mathcal{H}^{0}\left(F_{i+1}\right) \supset \ldots$ After choosing $R$-flat lifts and restricting to the special fibre, this yields a decreasing filtration of sheaves on $X$ concentrated in dimension zero, which stabilizes as well. Thus, $\mathcal{A}$ is artinian. The proof that $\mathcal{A}$ is noetherian is similar.

Similar arguments also prove that $\mathcal{O}_{\mathcal{X}_{K}}[1]$ is a minimal object (i.e. an object without proper subobjects) in $\mathcal{A}$ and therefore $\sigma$-stable of phase one. Indeed, if $0 \longrightarrow F \longrightarrow \mathcal{O}_{\mathcal{X}_{K}}[1] \longrightarrow G \longrightarrow 0$ is a decomposition in $\mathcal{A}$, then the long cohomology sequence shows $\mathcal{H}^{0}(G)=0$ and $\operatorname{rk}\left(\mathcal{H}^{-1}(F)\right)+$ $\operatorname{rk}\left(\mathcal{H}^{-1}(G)\right)=1$. Hence $\operatorname{rk}\left(\mathcal{H}^{-1}(G)\right)=0$, which would yield $G=0$, or $\operatorname{rk}\left(\mathcal{H}^{-1}(F)\right)=0$. The latter would result in a short exact sequence $0 \longrightarrow \mathcal{O}_{\mathcal{X}_{K}} \rightarrow \mathcal{H}^{-1}(G) \longrightarrow \mathcal{H}^{0}(F) \longrightarrow 0$ in $\mathbf{C o h}\left(\mathcal{X}_{K}\right)$ with $\mathcal{H}^{-1}(G)$ torsion free of rank one and $\mathcal{H}^{0}(F)$ torsion. As shown before, a torsion sheaf $\mathcal{H}^{0}(F)$ has Mukai vector $(0, s)$ with $s \geq 0$ and the Mukai vector of the torsion free rank one sheaf $\mathcal{H}^{-1}(G)$ is of the form $\left(1, s^{\prime}\right)$ with $s^{\prime} \leq 1$. The additivity of the Mukai vector leaves only the possibility $s=0$, which implies $F=0$ (see Example 2.11, iii)). Contradiction.

Suppose $F \in \mathcal{A}$ is a semi-rigid stable object with $\operatorname{End}_{\mathcal{X}_{K}}(F) \simeq K$. If we choose a lift $E \in \mathrm{D}^{\mathrm{b}}(\mathcal{X})$ of $F$ and denote the Mukai vector of $E_{0}$ by $(r, s)$, then $0=\chi_{K}(F, F)=\chi\left(E_{0}, E_{0}\right)=2 r s$ (2.2). Hence $r=0$ or $s=0$. On the other hand, $\chi_{K}\left(\mathcal{O}_{\mathcal{X}_{K}}, F\right)=r+s$ and since $\mathcal{O}_{\mathcal{X}_{K}}[1], F \in \mathcal{A}$ are both non-isomorphic stable objects of the same phase, $\operatorname{Ext}_{\mathcal{X}_{K}}^{i}\left(\mathcal{O}_{\mathcal{X}_{K}}, F\right) \neq 0$ at most for $i=0$ (use Serre duality for $i>1$ ). This shows $r+s \geq 0$. Thus, if $s=0$, then $r \geq 0$ and hence $r=0$, because objects in $\mathcal{A}$ have non-positive rank. Therefore, any semi-rigid stable $F \in \mathcal{A}$ with $\operatorname{End}_{\mathcal{X}_{K}}(F) \simeq K$ satisfies $r=0$, i.e. $F \in \operatorname{Coh}\left(\mathcal{X}_{K}\right)$, and, moreover, $F$ is torsion. Pick an $R$-flat lift $E$ of $F$, which is necessarily torsion as well. Proposition 2.10 shows that the support $\mathcal{Z} \subset \mathcal{X}$ of $E$ is of relative dimension zero over $\operatorname{Spf}(R)$. Clearly, the support of $E$ is irreducible, as otherwise $F$ would have a proper subsheaf contradicting the stability of $F$. The same argument shows that $E$ is a rank one sheaf on $\mathcal{Z}$. Hence, $F \simeq\left(\mathcal{O}_{\mathcal{Z}}\right)_{K}$, which is $K$-rational if and only if $\mathcal{Z} \subset \mathcal{X}$ is a section of $\pi: \mathcal{X} \rightarrow \operatorname{Spf}(R)$. Hence $F \simeq K(\mathbf{x})$ with End $_{\mathcal{X}_{K}}(K(\mathbf{x}))=K$.

2.7. Derived equivalences of the very general twistor fibre. Let us now consider two K3 surfaces $X$ and $X^{\prime}$, and formal deformations of them

$$
\pi: \mathcal{X} \longrightarrow \operatorname{Spf}(R) \text { and } \pi^{\prime}: \mathcal{X}^{\prime} \longrightarrow \operatorname{Spf}(R) \text {. }
$$


Moreover, we shall assume that $\pi: \mathcal{X} \rightarrow \operatorname{Spf}(R)$ is the formal twistor space of $X$ associated to a very general Kähler class $\omega$.

The aim of this section is to show that under the genericity assumption on the Kähler class any Fourier-Mukai equivalence between the general fibres $\mathcal{X}_{K}$ and $\mathcal{X}_{K}^{\prime}$ of the two formal deformations has, up to shift and spherical twist, a sheaf kernel.

Proposition 2.18. Suppose $\Phi: \mathrm{D}^{\mathrm{b}}\left(\mathcal{X}_{K}\right) \stackrel{\sim}{\rightarrow} \mathrm{D}^{\mathrm{b}}\left(\mathcal{X}_{K}^{\prime}\right)$ is a $K$-linear exact equivalence. Then, up to shift and spherical twist in $\mathcal{O}_{\mathcal{X}_{K}^{\prime}}$, the equivalence $\Phi$ identifies $K$-rational points of $\mathcal{X}_{K}$ with $K$-rational points of $\mathcal{X}_{K}^{\prime}$. More precisely, there exist integers $n$ and $m$ such that

$$
T_{K}^{n} \circ \Phi[m]:\left\{K(\mathbf{x}) \mid \mathbf{x} \in \mathcal{X}_{K} ; \operatorname{End}_{\mathcal{X}_{K}}(K(\mathbf{x})) \simeq K\right\} \stackrel{\sim}{\longrightarrow}\left\{K(\mathbf{y}) \mid \mathbf{y} \in \mathcal{X}_{K}^{\prime} ; \operatorname{End}_{\mathcal{X}_{K}}(K(\mathbf{y})) \simeq K\right\} .
$$

Proof. First note that although $\mathcal{X}_{K}^{\prime}$ is not necessarily a very general twistor fibre, Proposition 2.14 still applies. Indeed, the object $\mathcal{O}_{\mathcal{X}_{K}^{\prime}}$ is spherical and by Proposition 2.14 we know that up to shift $\mathcal{O}_{\mathcal{X}_{K}}$ is the only quasi-spherical object in $\mathrm{D}^{\mathrm{b}}\left(\mathcal{X}_{K}\right) \simeq \mathrm{D}^{\mathrm{b}}\left(\mathcal{X}_{K}^{\prime}\right)$. Since the property of being (quasi-)spherical is invariant under equivalence, one concludes that $\mathcal{O}_{\mathcal{X}_{K}}$ is mapped to a shift of $\mathcal{O}_{\mathcal{X}_{K}^{\prime}}$ and that $\mathcal{O}_{\mathcal{X}_{K}^{\prime}}$ is the only quasi-spherical object on $\mathrm{D}^{\mathrm{b}}\left(\mathcal{X}_{K}^{\prime}\right)$.

The argument follows literally the proof of [18, Lemma 4.9], so we will be brief. Let $\tilde{\sigma}$ be the stability condition on $\mathrm{D}^{\mathrm{b}}\left(\mathcal{X}_{K}^{\prime}\right)$ which is the image of $\sigma$ under $\Phi$. Then there exists an integer $n$, such that all sections $K(\mathbf{y})$ of $\mathcal{X}^{\prime} \rightarrow \operatorname{Spf}(R)$ are $T_{K}^{n}(\tilde{\sigma})$-stable (cf. Corollary 2.15] and [18, Prop. 2.18, Cor. 2.19]). In other words, for any $K$-rational point $\mathbf{y} \in \mathcal{X}_{K}^{\prime}$ the object $\Phi^{-1} T_{K}^{-n}(K(\mathbf{y}))$ is $\sigma$-stable. As $\Phi$ is an equivalence, $\Phi^{-1} T_{K}^{-n}(K(\mathbf{y}))$ is semi-rigid as well with $\operatorname{End}_{\mathcal{X}_{K}}\left(\Phi^{-1} T_{K}^{-n}(K(\mathbf{y}))\right)=K$. Hence, by Proposition 2.17 the set $\{K(\mathbf{y})\}$ is contained in $\left\{T_{K}^{n} \Phi(K(\mathbf{x}))[m]\right\}$ for some $m$.

Applying the same argument to $\Phi^{-1}$ yields equality $\left\{T^{n} \Phi(K(\mathbf{x})[m])\right\}=\{K(\mathbf{y})\}$.

Proposition 2.19. Suppose $\Phi: \mathrm{D}^{\mathrm{b}}\left(\mathcal{X}_{K}\right) \stackrel{\sim}{\rightarrow} \mathrm{D}^{\mathrm{b}}\left(\mathcal{X}_{K}^{\prime}\right)$ is a Fourier-Mukai equivalence with kernel $\mathcal{E}_{K} \in \mathrm{D}^{\mathrm{b}}\left(\left(\mathcal{X} \times_{R} \mathcal{X}^{\prime}\right)_{K}\right)$. If $\Phi$ induces a bijection of the $K$-rational points $\{K(\mathbf{x})\} \stackrel{\sim}{\longrightarrow}\left\{K\left(\mathbf{x}^{\prime}\right)\right\}$, then $\mathcal{E}_{K}$ is a sheaf, i.e. $\mathcal{E}_{K} \in \mathbf{C o h}\left(\left(\mathcal{X} \times{ }_{R} \mathcal{X}^{\prime}\right)_{K}\right)$.

Proof. The full triangulated subcategory $\mathcal{D} \subset \mathrm{D}^{\mathrm{b}}\left(\mathcal{X}_{K}\right) \simeq \mathrm{D}^{\mathrm{b}}\left(\mathbf{C o h}\left(\mathcal{X}_{K}\right)\right)$ of all complexes $F \in$ $\mathrm{D}^{\mathrm{b}}\left(\mathcal{X}_{K}\right)$ for which $\operatorname{Ext}_{\mathcal{X}_{K}}^{i}(F, K(\mathbf{x}))=0$ for all $i$ and all $K$-rational points $\mathbf{x} \in \mathcal{X}_{K}$ will play a central role in the proof.

i) We shall use the following general fact: Let $F \in \mathbf{C o h}\left(\mathcal{X}_{K}\right)$ such that $\operatorname{Hom}_{\mathcal{X}_{K}}(F, K(\mathbf{x}))=0$ for any $K$-rational point $\mathbf{x} \in \mathcal{X}_{K}$, then $\operatorname{Hom}_{\mathcal{X}_{K}}(K(\mathbf{x}), F)=0$ for any $K$-rational point $\mathbf{x} \in \mathcal{X}_{K}$. Moreover, in this case $F \in \mathcal{D}$.

In order to prove this, choose an $R$-flat lift $E$ of $F$. Then the support of $E$ is either $\mathcal{X}$ or a finite union $\bigcup \mathcal{Z}_{i}$ of irreducible multisections (see Proposition 2.10). In the first case we would have $\operatorname{Hom}_{\mathcal{X}_{K}}(F, K(\mathbf{x})) \neq 0$ for any point $\mathbf{x} \in \mathcal{X}_{K}$ (see Corollary 2.12, ii)), contradicting the assumption. Thus, $F \simeq \bigoplus F_{i}$ with each $\left(F_{i}\right)_{K}$ admitting a filtration with quotients isomorphic to $K\left(\mathbf{y}_{i}\right)$, where the $\mathbf{y}_{i}$ are points of the general fibre corresponding to different irreducible multisections (see Corollary 2.12 , i)). By our assumption, none of the points $\mathbf{y}_{i}$ can be $K$-rational. But then in fact $\operatorname{Hom}_{\mathcal{X}_{K}}\left(K(\mathbf{x}), K\left(\mathbf{y}_{i}\right)\right)=0$ for all $K$-rational points $\mathbf{x} \in \mathcal{X}_{K}$.

Since the $R$-flat lift $E$ of $F$ is supported in a finite union of multisection, the restriction $E_{0}$ of $E$ to the special fibre $X$ has rank zero. Hence $0=\operatorname{rk}\left(E_{0}\right)=\chi_{0}\left(k(x), E_{0}\right)=\chi_{K}(K(\mathbf{x}), F)=$ $-\operatorname{dim}_{K} \operatorname{Ext}_{\mathcal{X}_{K}}^{1}(K(\mathbf{x}), F)$, where $x \in X$ is the specialization of $K(\mathbf{x})$. This is the second assertion.

ii) Next we claim that if $F \in \mathcal{D}$, then all cohomology sheaves $\mathcal{H}^{q}(F) \in \mathbf{C o h}\left(\mathcal{X}_{K}\right)$ are as well contained in $\mathcal{D}$. Indeed, using the spectral sequence

$$
E_{2}^{p, q}=\operatorname{Ext}_{\mathcal{X}_{K}}^{p}\left(\mathcal{H}^{-q}(F), K(\mathbf{x})\right) \Rightarrow \operatorname{Ext}_{\mathcal{X}_{K}}^{p+q}(F, K(\mathbf{x}))
$$

one sees that for $q$ minimal with non-vanishing $\mathcal{H}^{-q}(F) \neq 0$ any non-trivial element in $E_{2}^{0, q}=$ $\operatorname{Hom}_{\mathcal{X}_{K}}\left(\mathcal{H}^{-q}(F), K(\mathbf{x})\right)$ would survive and thus contradict $F \in \mathcal{D}$. Hence, the maximal nontrivial cohomology sheaf of $F$ does not admit non-trivial homomorphisms to any $K$-rational point 
and is, therefore, due to i) contained in $\mathcal{D}$. Replacing $F$ by the cone of the natural morphism $F \rightarrow \mathcal{H}^{-q}(F)[q]$, which is again in $\mathcal{D}$ and with a smaller number of non-trivial cohomology sheaves, one can continue and eventually proves that all cohomology of $F$ is contained in $\mathcal{D}$.

iii) Consider a sheaf $0 \neq F \in \mathcal{D} \cap \mathbf{C o h}\left(\mathcal{X}_{K}\right)$. We claim that $\operatorname{Ext}_{\mathcal{X}_{K}}^{i}\left(\mathcal{O}_{\mathcal{X}_{K}}, F\right)=0$ for $i \neq 0$ and $\operatorname{Hom}_{\mathcal{X}_{K}}\left(\mathcal{O}_{\mathcal{X}_{K}}, F\right) \neq 0$.

By the definition of $\mathcal{D}$, one has $\chi_{K}(F, K(\mathbf{x}))=0$ for all $K$-rational points $\mathbf{x} \in \mathcal{X}_{K}$. Writing this as the Mukai pairing, one finds that the restriction $E_{0}$ of any $R$-flat lift $E$ of $F$ to the special fibre $X$ will be a sheaf with Mukai vector $(0,0, s)$, i.e. $E_{0}$ is a non-trivial sheaf concentrated in dimension zero. If $\operatorname{Ext}_{\mathcal{X}_{K}}^{i}\left(\mathcal{O}_{\mathcal{X}_{K}}, F\right) \neq 0$ for $i=1$ or $i=2$, then, by (2.3) , one would have $\operatorname{Ext}_{X}^{i}\left(\mathcal{O}_{X}, E_{0}\right) \neq 0$, which is absurd. On the other hand, since $s \neq 0$ for $F \neq 0$ (see Example 2.11, iii)), the Mukai pairing also shows $0 \neq s=\chi_{0}\left(\mathcal{O}_{X}, E_{0}\right)=\chi_{K}\left(\mathcal{O}_{\mathcal{X}_{K}}, F\right)$ and thus $\operatorname{Hom}_{\mathcal{X}_{K}}\left(\mathcal{O}_{\mathcal{X}_{K}}, F\right) \neq 0$.

iv) If $F \in \mathcal{D}$, then $\operatorname{Hom}_{\mathcal{X}_{K}}\left(\mathcal{O}_{\mathcal{X}_{K}}, \mathcal{H}^{q}(F)\right) \simeq \operatorname{Ext}_{\mathcal{X}_{K}}^{q}\left(\mathcal{O}_{\mathcal{X}_{K}}, F\right)$. Using ii) and iii), this follows from the spectral sequence

$$
E_{2}^{p, q}=\operatorname{Ext}_{\mathcal{X}_{K}}^{p}\left(\mathcal{O}_{\mathcal{X}_{K}}, \mathcal{H}^{q}(F)\right) \Rightarrow \operatorname{Ext}_{\mathcal{X}_{K}}^{p+q}\left(\mathcal{O}_{\mathcal{X}_{K}}, F\right) .
$$

v) Let us show that under $\Phi$ the image of any sheaf $F \in \mathbf{C o h}\left(\mathcal{X}_{K}\right)$ orthogonal to all $K$-rational points is again a sheaf $\Phi(F) \in \mathbf{C o h}\left(\mathcal{X}_{K}\right)$ (and moreover orthogonal to all $K$-rational points).

As all $K$-rational points are again of the form $\Phi(K(\mathbf{x}))$ for some $K$-rational point $\mathbf{x} \in \mathcal{X}_{K}$, the assumption $F \in \mathcal{D}$ implies $\Phi(F) \in \mathcal{D}^{\prime}$, where $\mathcal{D}^{\prime} \subset \mathrm{D}^{\mathrm{b}}\left(\mathcal{X}_{K}^{\prime}\right)$ is defined analogously to $\mathcal{D} \subset \mathrm{D}^{\mathrm{b}}\left(\mathcal{X}_{K}\right)$. Hence, using iii) and iv)

$$
\operatorname{Hom}_{\mathcal{X}_{K}^{\prime}}\left(\mathcal{O}_{\mathcal{X}_{K}^{\prime}}, \mathcal{H}^{q}(\Phi(F))\right)=\operatorname{Ext}_{\mathcal{X}_{K}^{\prime}}^{q}\left(\mathcal{O}_{\mathcal{X}_{K}^{\prime}}, \Phi(F)\right)=\operatorname{Ext}_{\mathcal{X}_{K}}^{q}\left(\mathcal{O}_{\mathcal{X}_{K}}, F\right)=0
$$

for $q \neq 0$. Here we use $\Phi\left(\mathcal{O}_{\mathcal{X}_{K}}\right) \simeq \mathcal{O}_{\mathcal{X}_{K}^{\prime}}$, which follows from Proposition 2.14 saying that $\mathcal{O}_{\mathcal{X}_{K}} \in$ $\mathrm{D}^{\mathrm{b}}\left(\mathcal{X}_{K}\right)$ respectively $\mathcal{O}_{\mathcal{X}_{K}^{\prime}} \in \mathrm{D}^{\mathrm{b}}\left(\mathcal{X}_{K}^{\prime}\right)$ are the only spherical objects up to shift. (The shift is indeed trivial which follows easily from the assumption $\Phi(K(\mathbf{x})) \simeq K\left(\mathbf{x}^{\prime}\right)$.) On the other hand, by ii), $\mathcal{H}^{q}(\Phi(F)) \in \mathcal{D}^{\prime}$ and thus by iii) $\operatorname{Hom}_{\mathcal{X}_{K}^{\prime}}\left(\mathcal{O}_{\mathcal{X}_{K}^{\prime}}, \mathcal{H}^{q}(\Phi(F))\right) \neq 0$ whenever $\mathcal{H}^{q}(\Phi(F)) \neq 0$. This yields $\Phi(F) \simeq \mathcal{H}^{0}(\Phi(F))$.

vi) We will now show that $\Phi$ not only sends $K$-rational points to $K$-rational points, but that in fact any point $K(\mathbf{y}), K$-rational or not, is mapped to a point. Applying the same argument to the inverse functor, one finds that $\Phi$ induces a bijection of the set of all ( $K$-rational or not) points.

If $K(\mathbf{y})$ is not $K$-rational, then $K(\mathbf{y}) \in \mathcal{D}$. Hence $G_{K}:=\Phi(K(\mathbf{y})) \in \mathcal{D}^{\prime} \cap \mathbf{C o h}\left(\mathcal{X}_{K}^{\prime}\right)$ due to v). Suppose $G \in \operatorname{Coh}\left(\mathcal{X}^{\prime}\right)$ is an $R$-flat lift of $G_{K}$. We shall argue as in i). Note that we can in fact apply Proposition 2.10 and Corollary 2.12, for $\mathcal{O}_{\mathcal{X}^{\prime}}$ is the only line bundle on $\mathcal{X}^{\prime}$ (otherwise there would be an extra spherical object) and Remark 2.13 therefore applies. The support of $G$ can either be $\mathcal{X}^{\prime}$ or a finite union of multisections. In the first case $\operatorname{Hom}_{K}\left(G_{K}, K\left(\mathbf{x}^{\prime}\right)\right) \neq 0$ for any $K$-rational point $\mathbf{x}^{\prime} \in \mathcal{X}_{K}^{\prime}$. As this would contradict $G_{K} \in \mathcal{D}^{\prime}$, we conclude that $G$ is supported on a finite union $\bigcup \mathcal{Z}_{i}$ of multisections $\mathcal{Z}_{i}$ each inducing a point $\mathbf{y}_{i} \in \mathcal{X}_{K}^{\prime}$. Thus, $G_{K} \simeq \bigoplus_{i=1}^{n} G_{i}$ with $G_{i}$ admitting a filtration with quotients isomorphic to $K\left(\mathbf{y}_{i}\right)$ (cf. Corollary 2.12, i)). Since $\Phi$ is an equivalence, $G_{K}$ is simple, i.e. End $\mathcal{X}_{K}\left(G_{K}\right)$ is a field. Thus, $n=1$ and $G_{K}=G_{1} \simeq K\left(\mathbf{y}_{1}\right)$.

vii) The last step is a standard argument. We have to show that the kernel of a Fourier-Mukai transform that sends points to points is a sheaf (cf. e.g. [17, Lemma 3.31]). If $\mathcal{E} \in \mathrm{D}^{\mathrm{b}}\left(\mathcal{X} \times_{R} \mathcal{X}^{\prime}\right)$ is a lift of $\mathcal{E}_{K}$, we have to show that the cohomology $\mathcal{H}^{q}(\mathcal{E})$ for $q \neq 0$ is $R$-torsion or equivalently that $\mathcal{H}^{q}(\mathcal{E})_{K}=0$ for $q \neq 0$.

Suppose $\mathcal{H}^{q}(\mathcal{E})$ is not $R$-torsion for some $q>0$. Let $q_{0}$ be maximal with this property and let $\mathbf{y} \in \mathcal{X}_{K}$ be a point corresponding to a multisection $\mathcal{Z} \subset \mathcal{X}$ in the image of the support of $\mathcal{H}^{q_{0}}(\mathcal{E})$ under the first projection. Then the sheaf pull-back $\mathcal{H}^{0}\left(i^{*} \mathcal{H}^{q_{0}}(\mathcal{E})\right)$ is non-trivial, where $i: \mathcal{Z} \times_{R} \mathcal{X}^{\prime} \rightarrow \mathcal{X} \times_{R} \mathcal{X}^{\prime}$ is the natural morphism. In fact, $\mathcal{H}^{0}\left(i^{*} \mathcal{H}^{q_{0}}(\mathcal{E})\right)_{K} \neq 0$. Consider the spectral sequence $\left(\right.$ in $\left.\mathrm{D}^{\mathrm{b}}\left(\mathcal{X} \times_{R} \mathcal{X}^{\prime}\right)\right)$ :

$$
E_{2}^{p, q}=\mathcal{H}^{p}\left(i^{*} \mathcal{H}^{q}(\mathcal{E})\right) \Rightarrow \mathcal{H}^{p+q}\left(i^{*} \mathcal{E}\right),
$$


which is concentrated in the region $p \leq 0$.

Due to the maximality of $q_{0}$, the non-vanishing $\mathcal{H}^{0}\left(i^{*} \mathcal{H}^{q_{0}}(\mathcal{E})\right)_{K} \neq 0$ implies $\mathcal{H}^{q_{0}}\left(i^{*} \mathcal{E}\right)_{K} \neq 0$. But then also $\mathcal{H}^{q_{0}}(\Phi(K(\mathbf{y})))=p_{\mathcal{X}_{K}^{\prime}}\left(\mathcal{H}^{q_{0}}\left(i^{*} \mathcal{E}\right)_{K}\right) \neq 0$, which contradicts vi) if $q_{0}>0$.

Suppose there exists a $q_{0}<0$ with $\mathcal{H}^{q_{0}}(\mathcal{E})_{K} \neq 0$. Choose $q_{0}<0$ maximal with this property and a multisection $\mathbf{y} \in \mathcal{X}_{K}$ in the support of (the direct image under the first projection of) $\mathcal{H}^{q_{0}}(\mathcal{E})$. Then $\mathcal{H}^{0}\left(i^{*} \mathcal{H}^{q_{0}}(\mathcal{E})\right)_{K} \neq 0$ and, by using the spectral sequence (2.6) again, $\mathcal{H}^{q_{0}}\left(i^{*} \mathcal{E}\right)_{K} \neq 0$. As above, this contradicts the assumption that $\Phi(K(\mathbf{y}))$ is a sheaf.

\section{Deformation of the Fourier-Mukai Kernel}

In this section we deal with the obstruction to deforming the kernel of a Fourier-Mukai equivalence sideways. To this end, we need to compare the Kodaira-Spencer classes of the two sides of the Fourier-Mukai equivalence. Before actually showing the triviality of the obstruction, in Section 3.2 we adapt various (known) facts about Hochschild (co)homology to our setting.

3.1. The obstructions. Let $X$ be a smooth projective variety and let $\pi_{n}: \mathcal{X}_{n} \rightarrow \operatorname{Spec}\left(R_{n}\right)$ be a scheme smooth and proper over $R_{n}$ such that $X \simeq \mathcal{X}_{n} \times_{R_{n}} \operatorname{Spec}\left(R_{0}\right)$. Assume that there exists a deformation $\pi_{n+1}: \mathcal{X}_{n+1} \longrightarrow \operatorname{Spec}\left(R_{n+1}\right)$ of $\mathcal{X}_{n}$ to $R_{n+1}$, i.e. a scheme smooth and proper over $R_{n+1}$ such that $\mathcal{X}_{n} \simeq \mathcal{X}_{n+1} \times_{R_{n+1}} \operatorname{Spec}\left(R_{n}\right)$. The extension class of the short exact sequence

$$
\left.0 \longrightarrow j_{n *} \mathcal{O}_{X} \stackrel{t^{n} d t}{\longrightarrow} \Omega_{\mathcal{X}_{n+1}}\right|_{\mathcal{X}_{n}} \longrightarrow \Omega_{\mathcal{X}_{n}} \longrightarrow 0
$$

is the (absolute) Kodaira-Spencer class $\widetilde{\kappa}_{n} \in \operatorname{Ext}_{\mathcal{X}_{n}}^{1}\left(\Omega_{\mathcal{X}_{n}}, \mathcal{O}_{X}\right)$.

The (absolute) Atiyah class of a complex $E_{n} \in \mathrm{D}^{\mathrm{b}}\left(\mathcal{X}_{n}\right)$ is by definition the class $\widetilde{A}\left(E_{n}\right) \in$ $\operatorname{Ext}_{\mathcal{X}_{n}}^{1}\left(E_{n}, E_{n} \otimes \Omega_{\mathcal{X}_{n}}\right)$ induced by the boundary map $\widetilde{\alpha}$ of the short exact sequence

$$
0 \longrightarrow J_{n} / J_{n}^{2} \stackrel{\mu_{1}}{\longrightarrow} \mathcal{O}_{\mathcal{X}_{n} \times \mathcal{X}_{n}} / J_{n}^{2} \longrightarrow \mathcal{O}_{\Delta_{n}} \longrightarrow 0,
$$

where $J_{n} \subset \mathcal{O}_{\mathcal{X}_{n} \times \mathcal{X}_{n}}$ is the ideal sheaf of the diagonal $\widetilde{\eta}_{n}: \mathcal{X}_{n} \stackrel{\sim}{\rightarrow} \Delta_{n} \subset \mathcal{X}_{n} \times \mathcal{X}_{n}$. (Note that the fibre products are not relative over $\operatorname{Spf}\left(R_{n}\right)$, but over $\operatorname{Spec}(\mathbb{C})$.) More precisely,

$$
\widetilde{A}\left(E_{n}\right): E_{n} \longrightarrow E_{n} \otimes \Omega_{\mathcal{X}_{n}}[1]
$$

is obtained by viewing the boundary map $\widetilde{\alpha}_{n}: \mathcal{O}_{\Delta_{n}} \rightarrow J_{n} / J_{n}^{2}[1] \simeq \widetilde{\eta}_{*} \Omega_{\mathcal{X}_{n}}[1]$ of (3.1) as a morphism between two Fourier-Mukai kernels and applying the induced functor transformation to $E_{n}$.

The Kodaira-Spencer class $\widetilde{\kappa}_{n}$ gives rise to a morphism $\operatorname{id}_{E_{n}} \otimes \widetilde{\kappa}_{n}: E_{n} \otimes \Omega_{\mathcal{X}_{n}}[1] \longrightarrow E_{n} \otimes j_{n *} \mathcal{O}_{X}[2]$, which then can be composed with the Atiyah class $\widetilde{A}\left(E_{n}\right)$ to give a class

$$
\widetilde{o}\left(E_{n}\right):=\widetilde{A}\left(E_{n}\right) \cdot \widetilde{\kappa}_{n} \in \operatorname{Ext}_{\mathcal{X}_{n}}^{2}\left(E_{n}, E_{n} \otimes\left(j_{n}\right)_{*} \mathcal{O}_{X}\right) \simeq \operatorname{Ext}_{X}^{2}\left(E_{0}, E_{0}\right),
$$

where the isomorphism is given by adjunction $j_{n}^{*} \dashv\left(j_{n}\right)_{*}$.

As it turns out, this class is the obstruction to deform $E_{n}$ sideways. This is

Theorem 3.1. Suppose $E_{n}$ is a perfect complex on $\mathcal{X}_{n}$ and the derived pull-back $E_{0}:=j_{n}^{*} E_{n}$ satisfies $\operatorname{Ext}_{X}^{0}\left(E_{0}, E_{0}\right) \simeq \mathbb{C}$ and $\operatorname{Ext}_{X}^{<0}\left(E_{0}, E_{0}\right)=0$. Then there exists $E_{n+1} \in \mathrm{D}_{\text {perf }}\left(\mathcal{X}_{n+1}\right)$ such that $i_{n}^{*} E_{n+1} \simeq E_{n}$ if and only if $\widetilde{o}\left(E_{n}\right)=0$.

The proof of the theorem, which makes use of Lieblich's obstruction in [25], can be found in [19, App. C]. When the deformation $\mathcal{X}_{n}$ is integrable, e.g. obtained as in Example 2.1, the theorem can also be deduced from the more general results in 22]. (The assumptions on the non-positive Ext's are not needed in [22].) Our main application of this theorem concerns the case where $E_{n}$ is a Fourier-Mukai kernel. In this situation the conditions on $E_{0}$ are easily verified.

Let us turn to the relative versions of $\widetilde{A}$ and $\widetilde{\kappa}_{n}$. The extension class

$$
\kappa_{n} \in \operatorname{Ext}_{\mathcal{X}_{n}}^{1}\left(\Omega_{\pi_{n}}, \mathcal{O}_{\mathcal{X}_{n}}\right)
$$


of the natural short exact sequence

$$
\left.0 \longrightarrow \mathcal{O}_{\mathcal{X}_{n}} \longrightarrow \Omega_{\mathcal{X}_{n+1}}\right|_{\mathcal{X}_{n}} \longrightarrow \Omega_{\pi_{n}} \longrightarrow 0,
$$

is the relative Kodaira-Spencer class of order $n$. Here $\Omega_{\pi_{n}}$ denotes the locally free sheaf of differentials over $R_{n}$.

The relative Atiyah class of a perfect complex $E_{n} \in \mathrm{D}_{\text {perf }}\left(\mathcal{X}_{n}\right)$ (cf. Remark 2.3) is the class

$$
A\left(E_{n}\right) \in \operatorname{Ext}_{\mathcal{X}_{n}}^{1}\left(E_{n}, E_{n} \otimes \Omega_{\pi_{n}}\right)
$$

that, as a morphism $E_{n} \longrightarrow E_{n} \otimes \Omega_{\mathcal{X}_{n}}[1]$, is induced by the morphism of Fourier-Mukai kernels

$$
\alpha_{n}: \mathcal{O}_{\Delta_{n}} \rightarrow\left(I_{n} / I_{n}^{2}\right)[1] \simeq \eta_{n *} \Omega_{\pi_{n}}[1] .
$$

Here $\eta_{n}: \mathcal{X}_{n} \stackrel{\sim}{\longrightarrow} \Delta_{n} \subset \mathcal{X}_{n} \times_{R_{n}} \mathcal{X}_{n}$ is the relative diagonal, $I_{n}$ its ideal sheaf, and $\alpha_{n}$ is the boundary morphism of the short exact sequence

$$
0 \longrightarrow I_{n} / I_{n}^{2} \longrightarrow \mathcal{O}_{\mathcal{X}_{n} \times_{R_{n}}} \mathcal{X}_{n} / I_{n}^{2} \longrightarrow \mathcal{O}_{\Delta_{n}} \longrightarrow 0 .
$$

The composition of $A\left(E_{n}\right)$ with id $\otimes \kappa_{n}[1]$ yields the relative obstruction class

$$
o\left(E_{n}\right):=A\left(E_{n}\right) \cdot \kappa_{n} \in \operatorname{Ext}_{\mathcal{X}_{n}}^{2}\left(E_{n}, E_{n}\right) .
$$

Moreover, the relative Atiyah class can be used to define the relative Chern character of a perfect complex in the usual way. (Clearly, for vector bundles or in fact arbitrary coherent sheaves on $X_{0}$ this coincides by Chern-Weil theory with the usual Chern character.) Composition in $\mathrm{D}_{\text {perf }}\left(\mathcal{X}_{n}\right)$ and exterior product $\Omega_{\pi_{n}}^{\otimes i} \rightarrow \Omega_{\pi_{n}}^{i}$ allow one to form

$$
\exp \left(A\left(E_{n}\right)\right) \in \bigoplus \operatorname{Ext}_{\mathcal{X}_{n}}^{i}\left(E_{n}, E_{n} \otimes \Omega_{\pi_{n}}^{i}\right)
$$

and taking the trace yields (use $E_{n}$ perfect)

$$
\operatorname{ch}\left(E_{n}\right):=\operatorname{tr}\left(\exp \left(A\left(E_{n}\right)\right)\right) \in \bigoplus H^{i}\left(\mathcal{X}_{n}, \Omega_{\pi_{n}}^{i}\right) .
$$

We compare now the absolute obstruction class $\widetilde{o}\left(E_{n}\right)$ with the relative one $o\left(E_{n}\right)$, in the situations of Theorem 3.1 .

Lemma 3.2. Let $E_{n}$ be a perfect complex on $\mathcal{X}_{n}$ as in Theorem 3.1 and assume furthermore that $E_{0}$ is rigid, i.e. $\operatorname{Ext}_{X}^{1}\left(E_{0}, E_{0}\right)=0$. Then $E_{n} \in \mathrm{D}_{\text {perf }}\left(\mathcal{X}_{n}\right)$ deforms to $E_{n+1} \in \mathrm{D}_{\text {perf }}\left(\mathcal{X}_{n+1}\right)$ if and only if the relative obstruction class $o\left(E_{n}\right) \in \operatorname{Ext}_{\mathcal{X}_{n}}^{2}\left(E_{n}, E_{n}\right)$ is trivial.

Proof. The image of the Atiyah class $\widetilde{A}\left(E_{n}\right)$ under the natural projection $\Omega_{\mathcal{X}_{n}} \rightarrow \Omega_{\pi_{n}}$ is the relative Atiyah class $A\left(E_{n}\right)$. Similarly, one can compare the two Kodaira-Spencer classes $\widetilde{\kappa}_{n} \in$ $\operatorname{Ext}_{\mathcal{X}_{n}}^{1}\left(\Omega_{\mathcal{X}_{n}}, \mathcal{O}_{X}\right)$ and $\kappa_{n} \in \operatorname{Ext}_{\mathcal{X}_{n}}^{1}\left(\Omega_{\pi_{n}}, \mathcal{O}_{\mathcal{X}}\right)$ by the following commutative diagram

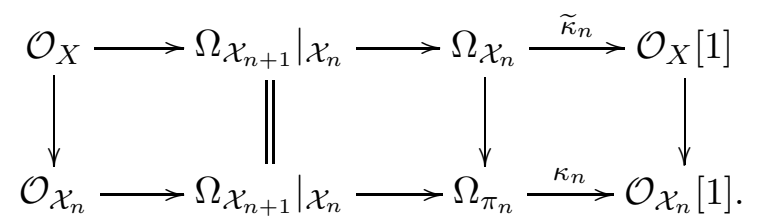

Here, $\left.\mathcal{O}_{X} \longrightarrow \mathcal{O}_{\mathcal{X}_{n}} \longrightarrow \Omega_{\mathcal{X}_{n+1}}\right|_{\mathcal{X}_{n}}$ is given by $1 \longmapsto t^{n} \longmapsto t^{n} d t$ and $\Omega_{\mathcal{X}_{n}} \rightarrow \Omega_{\pi_{n}}$ is the natural projection. Thus one obtains the following commutative diagram

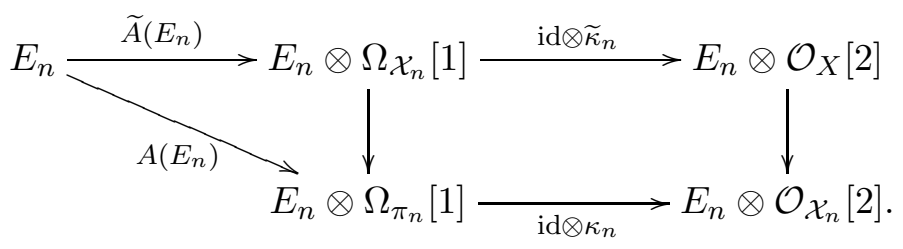


Let us consider the natural short exact sequence

$$
0 \longrightarrow \mathcal{O}_{X} \stackrel{t^{n}}{\longrightarrow} \mathcal{O}_{\mathcal{X}_{n}} \longrightarrow \mathcal{O}_{\mathcal{X}_{n-1}} \longrightarrow 0 .
$$

Tensoring it with $E_{n}$ and applying $\operatorname{Hom}_{\mathcal{X}_{n}}\left(E_{n},-\right)$ to it, one obtains the long exact sequence

$$
\cdots \longrightarrow \operatorname{Ext}_{\mathcal{X}_{n}}^{1}\left(E_{n}, E_{n-1}\right) \longrightarrow \operatorname{Ext}_{\mathcal{X}_{n}}^{2}\left(E_{n}, E_{0}\right) \stackrel{\varphi}{\longrightarrow} \operatorname{Ext}_{\mathcal{X}_{n}}^{2}\left(E_{n}, E_{n}\right) \longrightarrow \cdots
$$

and, by the previous discussion, $\varphi\left(\widetilde{o}\left(E_{n}\right)\right)=o\left(E_{n}\right)$.

By (2.3), $\operatorname{Ext}_{X}^{1}\left(E_{0}, E_{0}\right)=0$ implies $\operatorname{Ext}_{\mathcal{X}_{n}}^{1}\left(E_{n}, E_{n-1}\right) \simeq \operatorname{Ext}_{\mathcal{X}_{n-1}}^{1}\left(E_{n-1}, E_{n-1}\right)=0$. The claim follows.

3.2. Hochschild (co)homology and Fourier-Mukai transforms. Let $\pi_{n}: \mathcal{X}_{n} \rightarrow \operatorname{Spec}\left(R_{n}\right)$ be a smooth proper morphism with special fibre $X=\mathcal{X}_{0}$ of dimension $d$. We denote by $\eta_{n}$ : $\mathcal{X}_{n} \stackrel{\sim}{\longrightarrow} \Delta_{n} \subset \mathcal{X}_{n} \times_{R_{n}} \mathcal{X}_{n}$ the relative diagonal and define the relative Hochschild cohomology as the graded $R_{n}$-algebra

$$
\mathrm{HH}^{*}\left(\mathcal{X}_{n} / R_{n}\right):=\operatorname{Ext}_{\mathcal{X}_{n} \times_{R_{n}}}^{*} \mathcal{X}_{n}\left(\mathcal{O}_{\Delta_{n}}, \mathcal{O}_{\Delta_{n}}\right) \simeq \operatorname{Ext}_{\mathcal{X}_{n}}^{*}\left(\eta_{n}^{*} \mathcal{O}_{\Delta_{n}}, \mathcal{O}_{\mathcal{X}_{n}}\right)
$$

where the second isomorphism is obtained by adjunction. Multiplication $\mathrm{in}^{\mathrm{H}} \mathrm{HH}^{*}\left(\mathcal{X}_{n} / R_{n}\right)$ is given by composition in $\mathrm{D}^{\mathrm{b}}\left(\mathcal{X}_{n} \times_{R_{n}} \mathcal{X}_{n}\right)$ and the $R_{n}$-algebra structure is induced by the natural map $R_{n} \longrightarrow$ End $_{\mathcal{X}_{n} \times_{R_{n}} \mathcal{X}_{n}}\left(\mathcal{O}_{\Delta_{n}}\right)$.

Similarly, one defines the relative Hochschild homology as

$$
\mathrm{HH}_{*}\left(\mathcal{X}_{n} / R_{n}\right):=\operatorname{Ext}_{\mathcal{X}_{n} \times_{R_{n}}}^{-*} \mathcal{X}_{n}\left(\left(\eta_{n}\right)_{*}\left(\omega_{\pi_{n}}^{\check{2}}\right)[-d], \mathcal{O}_{\Delta_{n}}\right),
$$

which becomes a left $\mathrm{HH}^{*}\left(\mathcal{X}_{n} / R_{n}\right)$-module again via composition in $\mathrm{D}^{\mathrm{b}}\left(\mathcal{X}_{n} \times_{R_{n}} \mathcal{X}_{n}\right)$, where $\omega_{\pi_{n}}$ is the relative canonical bundle (see [35, 8, 5]). There is a natural isomorphism

$$
\mathrm{HH}_{k}\left(\mathcal{X}_{n} / R_{n}\right)=\operatorname{Ext}_{\mathcal{X}_{n} \times_{R_{n}} \mathcal{X}_{n}}^{-k}\left(\left(\eta_{n}\right)_{*}\left(\omega_{\pi_{n}}^{\check{2}}\right)[-d], \mathcal{O}_{\Delta_{n}}\right) \simeq \operatorname{Ext}_{\mathcal{X}_{n}}^{-k}\left(\mathcal{O}_{\mathcal{X}_{n}}, \eta_{n}^{*} \mathcal{O}_{\Delta_{n}}\right)=\operatorname{Tor}_{k}\left(\mathcal{O}_{\mathcal{X}_{n}}, \eta_{n}^{*} \mathcal{O}_{\Delta_{n}}\right) .
$$

This follows from the adjunction $\left(\eta_{n}\right) ! \dashv \eta_{n}^{*}$, where $\left(\eta_{n}\right) !: \mathrm{D}^{\mathrm{b}}\left(\mathcal{X}_{n}\right) \rightarrow \mathrm{D}^{\mathrm{b}}\left(\mathcal{X}_{n} \times_{R_{n}} \mathcal{X}_{n}\right)$ sends $E_{n}$ to $\left(\eta_{n}\right)_{*}\left(E_{n} \otimes \omega_{\pi_{n}}\right)[-d]$.

There is a natural isomorphism in $\mathrm{D}^{\mathrm{b}}\left(\mathcal{X}_{n}\right)$

$$
\mathbf{I}: \eta_{n}^{*} \mathcal{O}_{\Delta_{n}} \stackrel{\sim}{\longrightarrow} \bigoplus_{i} \Omega_{\pi_{n}}^{i}[i]
$$

called the (relative) Hochschild-Kostant-Rosenberg (HKR) isomorphism. The algebraic case was studied in [36, for the absolute case see also [28, 7]. The very general situation of an arbitrary analytic morphism has been discussed in [4, 5]. A characterization of $\mathbf{I}$ is given in Remark 3.4.

The HKR-isomorphism $\mathbf{I}$ is used in two different ways to define isomorphisms between Hochschild (co)homology and Dolbeault cohomology. Firstly, compose with the inverse of $\mathbf{I}$ in the first variable to obtain an isomorphism

$$
\operatorname{Ext}_{\mathcal{X}_{n}}^{*}\left(\eta_{n}^{*} \mathcal{O}_{\Delta_{n}}, \mathcal{O}_{\mathcal{X}_{n}}\right) \stackrel{\sim}{\longrightarrow} \operatorname{Ext}_{\mathcal{X}_{n}}^{*}\left(\bigoplus_{i} \Omega_{\pi_{n}}^{i}[i], \mathcal{O}_{\mathcal{X}_{n}}\right) \simeq \bigoplus_{i} H^{*-i}\left(\mathcal{X}_{n}, \bigwedge^{i} \mathcal{T}_{\pi_{n}}\right)
$$

where $\mathcal{T}_{\pi_{n}}$ is the relative tangent bundle. This leads to the HKR-isomorphism for Hochschild cohomology

$$
I^{\mathrm{HKR}}: \operatorname{Ext}_{\mathcal{X}_{n} \times_{R_{n}} \mathcal{X}_{n}}^{k}\left(\mathcal{O}_{\Delta_{n}}, \mathcal{O}_{\Delta_{n}}\right)=\operatorname{HH}^{k}\left(\mathcal{X}_{n} / R_{n}\right) \stackrel{\sim}{\longrightarrow} \operatorname{HT}^{k}\left(\mathcal{X}_{n} / R_{n}\right):=\bigoplus_{p+q=k} H^{p}\left(\mathcal{X}_{n}, \bigwedge^{q} \mathcal{T}_{\pi_{n}}\right) .
$$

For Hochschild homology we compose with $\mathbf{I}$ in the second variable to obtain:

$$
\operatorname{Ext}_{\mathcal{X}_{n}}^{-*}\left(\mathcal{O}_{\mathcal{X}_{n}}, \eta_{n}^{*} \mathcal{O}_{\Delta_{n}}\right) \stackrel{\sim}{\longrightarrow} \operatorname{Ext}_{\mathcal{X}_{n}}^{-*}\left(\mathcal{O}_{\mathcal{X}_{n}}, \bigoplus_{i} \Omega_{\pi_{n}}^{i}[i]\right) \simeq \bigoplus_{i} H^{-*+i}\left(\mathcal{X}_{n}, \Omega_{\pi_{n}}^{i}\right)
$$


This leads to the HKR-isomorphism

$$
I_{\mathrm{HKR}}: \operatorname{Ext}_{\mathcal{X}_{n} \times_{R_{n}} \mathcal{X}_{n}}^{-k}\left(\left(\eta_{n}\right)_{*}\left(\check{\omega}_{\pi_{n}}\right)[-d], \mathcal{O}_{\Delta_{n}}\right)=\mathrm{HH}_{k}\left(\mathcal{X}_{n} / R_{n}\right) \stackrel{\sim}{\longrightarrow} \mathrm{H} \Omega_{k}\left(\mathcal{X}_{n} / R_{n}\right):=\bigoplus_{q-p=k} H^{p}\left(\mathcal{X}_{n}, \Omega_{\pi_{n}}^{q}\right) .
$$

Remark 3.3. i) If $X$ is a smooth projective variety or a compact Kähler manifold, then the deformation invariance of the Hodge numbers together with the HKR-isomorphism $\mathrm{HH}_{*}\left(\mathcal{X}_{n} / R_{n}\right) \simeq$ $\mathrm{H} \Omega_{*}\left(\mathcal{X}_{n} / R_{n}\right)$ show that the $R_{n}$-module $\mathrm{HH}_{*}\left(\mathcal{X}_{n} / R_{n}\right)$ is free.

ii) Contraction defines on $\mathrm{H}_{*}\left(\mathcal{X}_{n} / R_{n}\right)$ the structure of a left $\operatorname{HT}^{*}\left(\mathcal{X}_{n} / R_{n}\right)$-module. The above isomorphism of $R_{n}$-modules

$$
\left(\mathrm{HH}^{*}\left(\mathcal{X}_{n} / R_{n}\right), \mathrm{HH}_{*}\left(\mathcal{X}_{n} / R_{n}\right)\right) \simeq\left(\mathrm{HT}^{*}\left(\mathcal{X}_{n} / R_{n}\right), \mathrm{H} \Omega_{*}\left(\mathcal{X}_{n} / R_{n}\right)\right)
$$

is expected to be a multiplicative isomorphism only after correcting $I^{\mathrm{HKR}}$ by $\operatorname{td}\left(\mathcal{T}_{\pi_{n}}\right)^{-1 / 2}$ and $I_{\mathrm{HKR}}$ by $\operatorname{td}\left(\mathcal{T}_{\pi_{n}}\right)^{1 / 2}$. See [24, 7, 10] for a discussion of the absolute case and [6, 9] for the ring structure of Hochschild cohomology.

Take $\pi_{n}^{\prime}: \mathcal{X}_{n}^{\prime} \longrightarrow \operatorname{Spec}\left(R_{n}\right)$ another smooth proper scheme over $R_{n}$ of relative dimension $d^{\prime}$ and set $X^{\prime}:=\mathcal{X}_{0}^{\prime}$. Let $\mathcal{E}_{n} \in \mathrm{D}_{\text {perf }}\left(\mathcal{X}_{n} \times_{R_{n}} \mathcal{X}_{n}^{\prime}\right)$ be a perfect complex defining a Fourier-Mukai transform

$$
\Phi_{\mathcal{E}_{n}}: \mathrm{D}^{\mathrm{b}}\left(\mathcal{X}_{n}\right) \stackrel{\sim}{\longrightarrow} \mathrm{D}^{\mathrm{b}}\left(\mathcal{X}_{n}^{\prime}\right)
$$

Following [8], $\Phi_{\mathcal{E}_{n}}$ induces a graded homomorphism

$$
\Phi_{\mathcal{E}_{n}}^{\mathrm{HH}_{*}}: \mathrm{HH}_{*}\left(\mathcal{X}_{n} / R_{n}\right) \longrightarrow \mathrm{HH}_{*}\left(\mathcal{X}_{n}^{\prime} / R_{n}\right) .
$$

For later use, we recall the construction in the relative setting.

Let $d=\operatorname{dim}(X)$ and $d^{\prime}=\operatorname{dim}\left(X^{\prime}\right)$. For two Fourier-Mukai kernels $\mathcal{E}$ and $\mathcal{F}$, we denote by $\mathcal{E} * \mathcal{F}$ the Fourier-Mukai kernel of the composition $\Phi_{\mathcal{E}} \circ \Phi_{\mathcal{F}}$ (see, e.g. [17, Prop. 5.10]). Define the functors

$$
\Psi_{1}: \mathrm{D}^{\mathrm{b}}\left(\mathcal{X}_{n} \times_{R_{n}} \mathcal{X}_{n}\right) \longrightarrow \mathrm{D}^{\mathrm{b}}\left(\mathcal{X}_{n} \times_{R_{n}} \mathcal{X}_{n}^{\prime}\right) \quad \Psi_{2}: \mathrm{D}^{\mathrm{b}}\left(\mathcal{X}_{n} \times_{R_{n}} \mathcal{X}_{n}^{\prime}\right) \longrightarrow \mathrm{D}^{\mathrm{b}}\left(\mathcal{X}_{n}^{\prime} \times_{R_{n}} \mathcal{X}_{n}^{\prime}\right)
$$

by $\Psi_{1}(\mathcal{G}):=\mathcal{E}_{n} * \mathcal{G}$ and $\Psi_{2}\left(\mathcal{G}^{\prime}\right):=\mathcal{G}^{\prime} *\left(\mathcal{E}_{n}\right)_{\mathrm{R}}$, where $\left(\mathcal{E}_{n}\right)_{\mathrm{R}}:=\mathcal{E}_{n}^{\prec} \otimes p_{1}^{*} \omega_{\pi_{n}}[d]$ denotes the FourierMukai kernel of the right adjoint of $\Phi_{\mathcal{E}_{n}}$ and $p_{1}$ is the projection (see e.g. [17, Prop. 5.9]). If, as above, $\Delta_{n} \subset \mathcal{X}_{n} \times_{R_{n}} \mathcal{X}_{n}$ and $\Delta_{n}^{\prime} \subset \mathcal{X}_{n}^{\prime} \times_{R_{n}} \mathcal{X}_{n}^{\prime}$ are the relative diagonals, then the composition $\Psi_{2} \circ \Psi_{1}$ induces a natural homomorphism

$$
\operatorname{Ext}_{\mathcal{X}_{n} \times_{R_{n}} \mathcal{X}_{n}}^{-*}\left(\left(\eta_{n}\right)_{*}\left(\omega_{\pi_{n}}^{\check{2}}\right)[-d], \mathcal{O}_{\Delta_{n}}\right) \longrightarrow \operatorname{Ext}_{\mathcal{X}_{n}^{\prime \prime} \times_{R_{n}} \mathcal{X}_{n}^{\prime}}^{-*}\left(\Psi_{2}\left(\Psi_{1}\left(\left(\eta_{n}\right)_{*}\left(\omega_{\pi_{n}}^{\check{2}}\right)\right)\right)[-d], \Psi_{2}\left(\Psi_{1}\left(\mathcal{O}_{\Delta_{n}}\right)\right)\right)
$$

An easy computation shows that $\Psi_{2}\left(\Psi_{1}\left(\mathcal{O}_{\Delta_{n}}\right)\right)=\mathcal{E}_{n} *\left(\mathcal{E}_{n}\right)_{\mathrm{R}}$ and $\Psi_{2}\left(\Psi_{1}\left(\left(\eta_{n}\right)_{*}\left(\omega_{\pi_{n}}^{\sim}\right)\right)\right)=\mathcal{E}_{n} *\left(\mathcal{E}_{n}\right)_{\mathrm{L}} \otimes$ $p_{2}^{*} \omega_{\pi_{n}^{\prime}}^{\curlyvee}\left[d-d^{\prime}\right]$, where $\left(\mathcal{E}_{n}\right)_{\mathrm{L}}:=\mathcal{E}_{n}^{\prec} \otimes p_{2}^{*} \omega_{\pi_{n}^{\prime}}\left[d^{\prime}\right]$ is the Fourier-Mukai kernel of the left adjoint to $\Phi_{\mathcal{E}_{n}}$ and $p_{2}$ is the projection.

The adjunction morphisms $\mathcal{E}_{n} *\left(\mathcal{E}_{n}\right)_{\mathrm{R}} \longrightarrow \mathcal{O}_{\Delta_{n}^{\prime}}$ and $\mathcal{O}_{\Delta_{n}^{\prime}} \rightarrow \mathcal{E}_{n} *\left(\mathcal{E}_{n}\right)_{\mathrm{L}}$ (see [8]) lead to natural morphisms

$$
\xi_{\mathrm{R}}: \Psi_{2}\left(\Psi_{1}\left(\mathcal{O}_{\Delta_{n}}\right)\right) \longrightarrow \mathcal{O}_{\Delta_{n}^{\prime}} \text { and } \xi_{\mathrm{L}}:\left(\eta_{n}^{\prime}\right)_{*}\left(\omega_{\pi_{n}^{\prime}}^{\check{2}}\right)\left[-d^{\prime}\right] \longrightarrow \Psi_{2}\left(\Psi_{1}\left(\left(\eta_{n}\right)_{*}\left(\omega_{\pi_{n}}^{\check{2}}\right)\right)\right)[-d]
$$

(which are isomorphisms if $\Phi_{\mathcal{E}_{n}}$ is an equivalence). Composition with both morphisms eventually leads to

$$
\Phi_{\mathcal{E}_{n}}^{\mathrm{HH}_{*}}: \mathrm{HH}_{*}\left(\mathcal{X}_{n} / R_{n}\right) \longrightarrow \operatorname{Ext}_{\mathcal{X}_{n}^{\prime} \times R_{n}}^{-*} \mathcal{X}_{n}^{\prime}\left(\left(\eta_{n}^{\prime}\right)_{*}\left({\check{\pi_{n}^{\prime}}}_{\check{2}}^{\check{2}}\right)\left[-d^{\prime}\right], \mathcal{O}_{\Delta_{n}^{\prime}}\right)=\mathrm{HH}_{*}\left(\mathcal{X}_{n}^{\prime} / R_{n}\right)
$$

It is easy to show, using the construction, that the action on Hochschild homology is functorial.

If $\Phi_{\mathcal{E}_{n}}$ is an equivalence, then it induces an isomorphism at the level of Hochschild cohomology as well. Hence we have a pair of isomorphisms

$$
\left(\Phi_{\mathcal{E}_{n}}^{\mathrm{HH}^{*}}, \Phi_{\mathcal{E}_{n}}^{\mathrm{HH}_{*}}\right):\left(\mathrm{HH}^{*}\left(\mathcal{X}_{n} / R_{n}\right), \mathrm{HH}_{*}\left(\mathcal{X}_{n} / R_{n}\right)\right) \stackrel{\sim}{\longrightarrow}\left(\mathrm{HH}^{*}\left(\mathcal{X}_{n}^{\prime} / R_{n}\right), \mathrm{HH}_{*}\left(\mathcal{X}_{n}^{\prime} / R_{n}\right)\right),
$$


with $\Phi_{\mathcal{E}_{n}}^{\mathrm{HH}^{*}}$ an isomorphism of $R_{n}$-algebras and $\Phi_{\mathcal{E}_{n}}^{\mathrm{HH}_{*}}$ compatible with the $\mathrm{HH}^{*}$-module structure on both sides (this is a simple generalization to the relative setting of e.g. [17, Rmk. 6.3]).

Conjugating with the HKR-isomorphisms yields also isomorphisms

$$
\Phi_{\mathcal{E}_{n}}^{\mathrm{HT}^{*}}:=I^{\mathrm{HKR}} \circ \Phi_{\mathcal{E}_{n}}^{\mathrm{HH}^{*}} \circ\left(I^{\mathrm{HKR}}\right)^{-1}: \operatorname{HT}^{*}\left(\mathcal{X}_{n} / R_{n}\right) \stackrel{\sim}{\longrightarrow} \mathrm{HT}^{*}\left(\mathcal{X}_{n}^{\prime} / R_{n}\right)
$$

and

$$
\Phi_{\mathcal{E}_{n}}^{\mathrm{H} \Omega_{*}}:=I_{\mathrm{HKR}} \circ \Phi_{\mathcal{E}_{n}}^{\mathrm{HH}} \circ I_{\mathrm{HKR}}^{-1}: \mathrm{H} \Omega_{*}\left(\mathcal{X}_{n} / R_{n}\right) \stackrel{\sim}{\rightarrow} \mathrm{H} \Omega_{*}\left(\mathcal{X}_{n}^{\prime} / R_{n}\right),
$$

which, however, are in general not compatible with the natural multiplicative structure of the pair $\left(\mathrm{HT}^{*}, \mathrm{H} \Omega_{*}\right)$. Also note that $\Phi_{\mathcal{E}_{n}}^{\mathrm{HT}}$ and $\Phi_{\mathcal{E}_{n}}^{\mathrm{H} \Omega_{*}}$ are graded, but often not bigraded.

Finally we recall the definition of the Hochschild versions of the Atiyah class and the Chern character (see [5, 17] and the original [28]). Consider the adjunction morphism adj : $\mathcal{O}_{\Delta_{n}} \longrightarrow\left(\eta_{n}\right)_{*} \eta_{n}^{*} \mathcal{O}_{\Delta_{n}}$ as a morphism between Fourier-Mukai kernels. The associated morphism between the FourierMukai transforms applied to $E_{n} \in \mathrm{D}^{\mathrm{b}}\left(\mathcal{X}_{n}\right)$ yields the Hochschild Atiyah class

$$
A H\left(E_{n}\right): E_{n} \longrightarrow E_{n} \otimes \eta_{n}^{*} \mathcal{O}_{\Delta_{n}} .
$$

Next one defines the Hochschild Chern character of a perfect complex $E_{n} \in \mathrm{D}_{\text {perf }}\left(\mathcal{X}_{n}\right)$ as

$$
\operatorname{ch}^{\mathrm{HH}_{*}}\left(E_{n}\right):=\operatorname{tr}\left(A H\left(E_{n}\right)\right) \in \operatorname{Hom}\left(\mathcal{O}_{\mathcal{X}_{n}}, \eta_{n}^{*} \mathcal{O}_{\Delta_{n}}\right) \simeq \mathrm{HH}_{0}\left(\mathcal{X}_{n} / R_{n}\right) .
$$

Remark 3.4. i) The HKR-isomorphism $I$ in (3.2) can also be described in terms of the universal relative Atiyah class (see [5, 7]). Indeed, let $\alpha_{n}: \mathcal{O}_{\Delta_{n}} \longrightarrow\left(\eta_{n}\right)_{*} \Omega_{\pi_{n}}[1]$ be the universal relative Atiyah class and denote by

$$
\exp \left(\alpha_{n}\right): \mathcal{O}_{\Delta_{n}} \rightarrow \bigoplus_{i}\left(\eta_{n}\right)_{*} \Omega_{\pi_{n}}^{i}[i]
$$

its exponential. The push-forward of $\mathbf{I}$ composed with the adjunction map equals $\exp \left(\alpha_{n}\right)$, i.e.

$$
\exp \left(\alpha_{n}\right): \mathcal{O}_{\Delta_{n}} \stackrel{\text { adj }}{\longrightarrow}\left(\eta_{n}\right)_{*} \eta_{n}^{*} \mathcal{O}_{\Delta_{n}} \stackrel{\left(\eta_{n}\right)_{*} \mathbf{I}}{\longrightarrow}\left(\eta_{n}\right)_{*}\left(\bigoplus_{i} \Omega_{\pi_{n}}^{i}[i]\right) \text {. }
$$

In other words, under the isomorphism

$$
\operatorname{Hom}_{\mathcal{X}_{n}}\left(\eta_{n}^{*} \mathcal{O}_{\Delta_{n}}, \bigoplus_{i} \Omega_{\pi_{n}}^{i}[i]\right) \simeq \operatorname{Hom}_{\mathcal{X}_{n} \times_{R_{n}} \mathcal{X}_{n}}\left(\mathcal{O}_{\Delta_{n}},\left(\eta_{n}\right)_{*}\left(\bigoplus_{i} \Omega_{\pi_{n}}^{i}[i]\right)\right)
$$

given by adjunction the HKR-isomorphism $\mathbf{I}$ is mapped to $\exp \left(\alpha_{n}\right)$.

Using this description of $\mathbf{I}$, one sees that $A H\left(E_{n}\right)$ and $A\left(E_{n}\right)$ are related by

$$
\left(\operatorname{id}_{E_{n}} \otimes \mathbf{I}\right) \circ A H\left(E_{n}\right)=\exp \left(A\left(E_{n}\right)\right) .
$$

(There is a small difference between $\exp \left(A\left(E_{n}\right)\right.$ ) and the Atiyah-Chern character $A C\left(E_{n}\right)=$ $\exp \left(-A\left(E_{n}\right)\right)$ in [5], which is due to a different sign convention in the definition of the Atiyah class. It is of no importance for our discussion.)

ii) Taking traces of (3.4) one obtains

$$
I_{\mathrm{HKR}}\left(\operatorname{ch}^{\mathrm{HH}_{*}}\left(E_{n}\right)\right)=\operatorname{ch}\left(E_{n}\right)
$$

for all perfect complexes $E_{n} \in \mathrm{D}_{\text {perf }}\left(\mathcal{X}_{n}\right)$. For the absolute case see e.g. [7].

Consider a perfect complex $E_{n} \in \mathrm{D}_{\text {perf }}\left(\mathcal{X}_{n}\right)$ as a Fourier-Mukai kernel on $\mathcal{X}_{n} \simeq \operatorname{Spec}\left(R_{n}\right) \times_{R_{n}} \mathcal{X}_{n}$. The induced Fourier-Mukai transform $\Phi_{E_{n}}: \mathrm{D}^{\mathrm{b}}\left(\operatorname{Spec}\left(R_{n}\right)\right) \rightarrow \mathrm{D}^{\mathrm{b}}\left(\mathcal{X}_{n}\right)$ yields

$$
\Phi_{E_{n}}^{\mathrm{HH}_{*}}: \mathrm{HH}_{0}\left(\operatorname{Spec}\left(R_{n}\right) / R_{n}\right) \longrightarrow \mathrm{HH}_{0}\left(\mathcal{X}_{n} / R_{n}\right),
$$

where, by definition, $\mathrm{HH}_{0}\left(\operatorname{Spec}\left(R_{n}\right) / R_{n}\right)=\operatorname{End}_{R_{n}}\left(R_{n}\right)$. 
Lemma 3.5. Let $\Phi_{\mathcal{E}_{n}}: \mathrm{D}^{\mathrm{b}}\left(\mathcal{X}_{n}\right) \rightarrow \mathrm{D}^{\mathrm{b}}\left(\mathcal{X}_{n}^{\prime}\right)$ be a Fourier-Mukai functor with $\mathcal{E}_{n} \in \mathrm{D}_{\text {perf }}\left(\mathcal{X}_{n} \times_{R_{n}} \mathcal{X}_{n}^{\prime}\right)$ and let $E_{n} \in \mathrm{D}_{\text {perf }}\left(\mathcal{X}_{n}\right)$. Then

i) $\operatorname{ch}^{\mathrm{HH}_{*}}\left(E_{n}\right)=\Phi_{E_{n}}^{\mathrm{HH}_{*}}(1)$;

ii) $\Phi_{\mathcal{E}_{n}}^{\mathrm{HH}_{*}}\left(\operatorname{ch}^{\mathrm{HH}_{*}}\left(E_{n}\right)\right)=\operatorname{ch}^{\mathrm{HH}_{*}}\left(\Phi_{\mathcal{E}_{n}}\left(E_{n}\right)\right)$.

Proof. To prove part i), one first observes that $\Psi_{2} \circ \Psi_{1}: \operatorname{End}_{R_{n}}\left(R_{n}\right) \rightarrow \operatorname{End}_{R_{n}}\left(\Psi_{2}\left(E_{n}\right)\right)$ sends

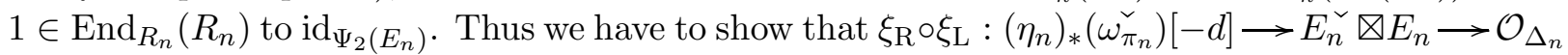
equals $\operatorname{tr}\left(A H\left(E_{n}\right)\right)$ under the adjunction

$$
\operatorname{Hom}_{\mathcal{X}_{n} \times_{R_{n}} \mathcal{X}_{n}}\left(\left(\eta_{n}\right)_{*}\left(\omega_{\pi_{n}}^{\check{r}}\right)[-d], \mathcal{O}_{\mathcal{X}_{n}}\right) \simeq \operatorname{Hom}_{\mathcal{X}_{n}}\left(\mathcal{O}_{\mathcal{X}_{n}}, \eta_{n}^{*} \mathcal{O}_{\Delta_{n}}\right) .
$$

To see this, observe that $\xi_{\mathrm{L}}$ under the adjunction

$$
\operatorname{Hom}_{\mathcal{X}_{n} \times_{R_{n}} \mathcal{X}_{n}}\left(\left(\eta_{n}\right)_{*}\left(\left(_{\pi_{n}}^{\curlyvee}\right)[-d], E_{n}^{\curlyvee} \otimes E_{n}\right) \simeq \operatorname{Hom}_{\mathcal{X}_{n}}\left(\mathcal{O}_{\mathcal{X}_{n}}, \eta_{n}^{*}\left(E_{n}^{\ulcorner} \otimes E_{n}\right)\right)\right.
$$

corresponds to the identity section of $\mathcal{E} n d\left(E_{n}\right)$ and that $\eta_{n}^{*} \xi_{\mathrm{R}} \in \operatorname{Hom}_{\mathcal{X}_{n}}\left(\mathcal{E} n d\left(E_{n}\right), \eta_{n}^{*} \mathcal{O}_{\Delta_{n}}\right)$ is obtained by composing the pull-back of the natural adjunction morphism

$$
\eta_{n}^{*}\left(E_{n}^{\curlyvee} \otimes E_{n} \longrightarrow\left(\eta_{n}\right)_{*} \eta_{n}^{*}\left(E_{n}^{\prec} \otimes E_{n}\right)\right)
$$

with $\eta_{n}^{*}\left(\eta_{n}\right)_{*}(\operatorname{tr}): \eta_{n}^{*}\left(\eta_{n}\right)_{*} \mathcal{E} n d\left(E_{n}\right) \longrightarrow \eta_{n}^{*} \mathcal{O}_{\Delta_{n}}$. Both assertions follow from the construction of the left and right adjoint of a Fourier-Mukai functor. In particular, $\xi_{\mathrm{R}}$ is indeed the composition of the restriction $E_{n}^{\curlyvee} \otimes E_{n} \longrightarrow\left(\eta_{n}\right)_{*} \mathcal{E} n d\left(E_{n}\right)$ with $\left(\eta_{n}\right)_{*}(\operatorname{tr})$. To conclude, recall that $A H\left(E_{n}\right)$ is obtained by applying adj : $\mathcal{O}_{\Delta_{n}} \longrightarrow\left(\eta_{n}\right)_{*} \eta_{n}^{*} \mathcal{O}_{\Delta_{n}}$, viewed as a morphism of Fourier-Mukai kernels, to $E_{n}$. Hence $\operatorname{ch}^{\mathrm{HH}_{*}}\left(E_{n}\right)=\operatorname{tr}\left(A H\left(E_{n}\right)\right)$ can be described as the composition of the identity $\mathcal{O}_{\mathcal{X}_{n}} \longrightarrow \mathcal{E} n d\left(E_{n}\right)$ with adj applied to $\mathcal{E} n d\left(E_{n}\right)$ :

$$
\mathcal{E} n d\left(E_{n}\right) \longrightarrow \mathcal{E} n d\left(E_{n}\right) \otimes \eta_{n}^{*} \mathcal{O}_{\Delta_{n}}
$$

followed by the trace on $\mathcal{E} n d\left(E_{n}\right)$. Then note that (3.7) clearly equals (3.6), which yields the equality in i).

Part ii) is the relative version of [8, Thm. 10] and follows directly from i) and functoriality.

Notice that the right hand side in part i) of the previous lemma is taken as the definition of the Hochschild Chern character in the absolute case in [8].

3.3. Controlling the obstructions. Let $\mathcal{X}_{n}$ and $\mathcal{X}_{n}^{\prime}$ be as in the previous section. Take $\mathcal{E}_{n} \in$ $\mathrm{D}_{\text {perf }}\left(\mathcal{X}_{n} \times_{R_{n}} \mathcal{X}_{n}^{\prime}\right)$ be the kernel of a Fourier-Mukai equivalence $\Phi_{\mathcal{E}_{n}}: \mathrm{D}^{\mathrm{b}}\left(\mathcal{X}_{n}\right) \rightarrow \mathrm{D}^{\mathrm{b}}\left(\mathcal{X}_{n}^{\prime}\right)$. Let $v_{n} \in H^{1}\left(\mathcal{X}_{n}, \mathcal{T}_{\pi_{n}}\right) \subset \operatorname{HT}^{2}\left(\mathcal{X}_{n} / R_{n}\right)$ and suppose $v_{n}^{\prime}:=\Phi_{\mathcal{E}_{n}}^{\mathrm{HT}^{*}}\left(v_{n}\right) \in H^{1}\left(\mathcal{X}_{n}^{\prime}, \mathcal{T}_{\pi_{n}^{\prime}}\right) \subset \operatorname{HT}^{2}\left(\mathcal{X}_{n} / R_{n}\right)$. The inverse images of both classes to the product $\mathcal{X}_{n} \times_{R_{n}} \mathcal{X}_{n}^{\prime}$ can be considered as classes in $H^{1}\left(\mathcal{X}_{n} \times_{R_{n}} \mathcal{X}_{n}^{\prime}, \mathcal{T}_{\pi_{n} \times \pi_{n}^{\prime}}\right) \subset \operatorname{HT}^{2}\left(\mathcal{X}_{n} \times_{R_{n}} \mathcal{X}_{n}^{\prime} / R_{n}\right)$. We write $p_{1}^{*} v_{n}+p_{2}^{*} v_{n}^{\prime}$ as $v_{n} \boxplus v_{n}^{\prime} \in H^{1}\left(\mathcal{X}_{n} \times_{R_{n}}\right.$ $\left.\mathcal{X}_{n}^{\prime}, \mathcal{T}_{\pi_{n} \times \pi_{n}^{\prime}}\right)$.

For any $w \in \operatorname{HT}^{*}\left(\mathcal{X}_{n} \times_{R_{n}} \mathcal{X}_{n}^{\prime} / R_{n}\right)$ contraction with the exponential of the relative Atiyah class of $\mathcal{E}_{n}$ defines a class $\exp \left(A\left(\mathcal{E}_{n}\right)\right) \cdot w \in \operatorname{Ext}_{\mathcal{X}_{n} \times_{R_{n}} \mathcal{X}_{n}^{\prime}}\left(\mathcal{E}_{n}, \mathcal{E}_{n}\right)$. Applied to $v_{n} \boxplus v_{n}^{\prime}$ the component in $\operatorname{Ext}_{\mathcal{X}_{n} \times_{R_{n}} \mathcal{X}_{n}^{\prime}}^{2}\left(\mathcal{E}_{n}, \mathcal{E}_{n}\right)$ is simply the contraction with the relative Atiyah class.

The following is a straightforward generalization of a result of Toda [34] which itself relies on Căldăraru's paper [7]. For the reader's convenience we will sketch the main arguments of the proof.

Lemma 3.6. With the above assumptions, one has

$$
0=A\left(\mathcal{E}_{n}\right) \cdot\left(v_{n} \boxplus v_{n}^{\prime}\right) \in \operatorname{Ext}_{\mathcal{X}_{n} \times_{R_{n}} \mathcal{X}_{n}^{\prime}}^{2}\left(\mathcal{E}_{n}, \mathcal{E}_{n}\right) .
$$

Proof. This results relies on the commutativity of the following diagram (see [34, Lemma 5.7,5.8]) which in turn is based upon the description of the HKR-isomorphisms in terms of the universal 
Atiyah class (see (3.3)):

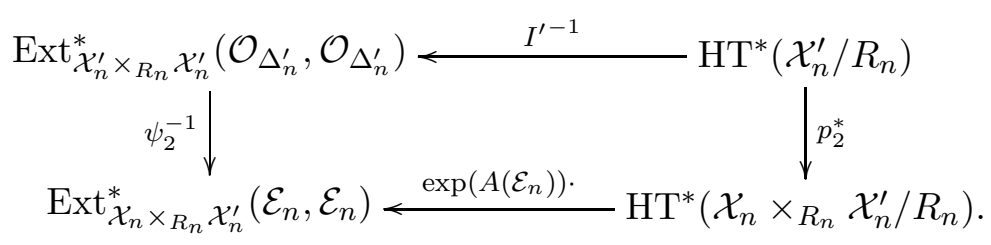

(Here, $\psi_{2}$ is induced by the equivalence $\Psi_{2}$ in Section 3.2. Similarly, $\psi_{1}$ further below is induced by $\Psi_{1}$.) A similar diagram holds true with $\mathcal{X}_{n}^{\prime}$ replaced by $\mathcal{X}_{n}$ and pulling back via the first projection. Then one has to add the pull-back $\tau^{*}$ of the automorphism $\tau$ of $\mathcal{X}_{n} \times_{R_{n}} \mathcal{X}_{n}$ interchanging the two factors. As we will see, the appearance of $\tau^{*}$ is crucial. One obtains the commutative diagram

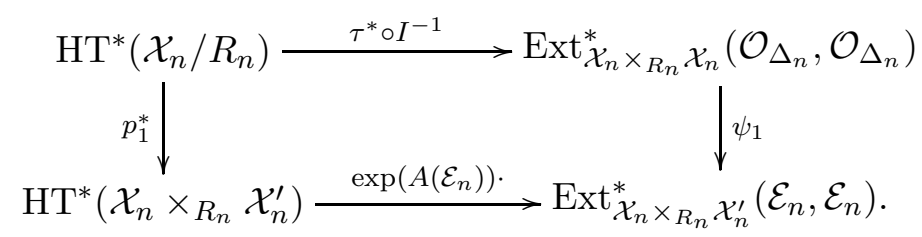

The proof given in 34] generalizes in a straightforward way.

The action of $\tau^{*}$ can be best understood via the isomorphism $\operatorname{HT}^{*}\left(\mathcal{X}_{n} / R_{n}\right) \simeq \mathrm{HH}^{*}\left(\mathcal{X}_{n} / R_{n}\right)=$ $\operatorname{Ext}_{\mathcal{X}_{n} \times_{R_{n}} \mathcal{X}_{n}}^{*}\left(\mathcal{O}_{\Delta_{n}}, \mathcal{O}_{\Delta_{n}}\right)$. It turns out that $\tau^{*}$ respects the bigrading of $\operatorname{HT}^{*}\left(\mathcal{X}_{n} / R_{n}\right)$ and acts by $(-1)^{q}$ on $H^{p}\left(\mathcal{X}_{n}, \bigwedge^{q} \mathcal{T}_{\pi_{n}}\right)$.

The degree two component of the product of a class in $H^{1}\left(\mathcal{X}_{n} \times_{R_{n}} \mathcal{X}_{n}^{\prime}, \mathcal{T}_{\pi_{n} \times \pi_{n}^{\prime}}\right)$ with $\exp \left(A\left(\mathcal{E}_{n}\right)\right)$ is simply the contraction with the Atiyah class. Thus one finds

$$
\begin{aligned}
A\left(\mathcal{E}_{n}\right) \cdot\left(v_{n} \boxplus v_{n}^{\prime}\right) & =A\left(\mathcal{E}_{n}\right) \cdot p_{1}^{*} v_{n}+A\left(\mathcal{E}_{n}\right) \cdot p_{2}^{*} v_{n}^{\prime} \\
& =\psi_{1}\left(\tau^{*}\left(I^{-1}\left(v_{n}\right)\right)\right)+\psi_{2}^{-1}\left(I^{\prime-1}\left(v_{n}^{\prime}\right)\right) \\
& =-\psi_{1}\left(I^{-1}\left(v_{n}\right)\right)+\psi_{2}^{-1}\left(I^{\prime-1}\left(v_{n}^{\prime}\right)\right) \\
& =\psi_{2}^{-1} I^{\prime-1}\left(-I^{\prime}\left(\psi_{2}\left(\psi_{1}\left(I^{-1}\left(v_{n}\right)\right)\right)+v_{n}^{\prime}\right)\right. \\
& =\psi_{2}^{-1} I^{\prime-1}\left(-\Phi_{\mathcal{E}_{n}}^{\mathrm{HT}^{2}}\left(v_{n}\right)+v_{n}^{\prime}\right)=0
\end{aligned}
$$

which is precisely what we claimed.

Combining this result and Lemma 3.2, we have the following:

Proposition 3.7. With the above assumptions, suppose furthermore that

i) $\mathcal{E}_{n} \in \mathrm{D}_{\text {perf }}\left(\mathcal{X}_{n} \times_{R_{n}} \mathcal{X}_{n}^{\prime}\right)$ is rigid;

ii) $v_{n} \boxplus v_{n}^{\prime} \in H^{1}\left(\mathcal{X}_{n} \times_{R_{n}} \mathcal{X}_{n}^{\prime}, \mathcal{T}_{\pi_{n} \times \pi_{n}^{\prime}}\right)$ is the relative Kodaira-Spencer class $\kappa_{n}$ corresponding to some deformation $\mathcal{Y}_{n+1} \longrightarrow \operatorname{Spec}\left(R_{n+1}\right)$ of $\mathcal{X}_{n} \times R_{n} \mathcal{X}_{n}^{\prime} \rightarrow \operatorname{Spec}\left(R_{n}\right)$.

Then there exists a perfect complex $\mathcal{E}_{n+1} \in \mathrm{D}_{\text {perf }}\left(\mathcal{Y}_{n+1}\right)$ such that $\mathcal{E}_{n} \simeq L i_{n}^{*} \mathcal{E}_{n+1}$.

In the next example we will see an explicit case in which assumption ii) of the previous result can be controlled.

Example 3.8. Let $\pi: \mathbb{X} \rightarrow D$ be a smooth proper analytic family over a 1-dimensional disk $D$ with distinguished fibre $X:=\pi^{-1}(0)$. We assume that the Kodaira-Spencer map, i.e. the boundary map of the tangent bundle sequence $0 \longrightarrow \mathcal{T}_{\pi} \longrightarrow \mathcal{T}_{\mathbb{X}} \longrightarrow \pi^{*} \mathcal{T}_{D} \longrightarrow 0$, yields an isomorphism $\kappa: \mathcal{T}_{D} \stackrel{\sim}{\longrightarrow} R^{1} \pi_{*} \mathcal{T}_{\pi} \simeq \mathcal{E} x t_{\pi}^{1}\left(\Omega_{\pi}, \mathcal{O}_{\mathbb{X}}\right)$ and that $h^{1}\left(\mathbb{X}_{t}, \mathcal{T}_{\mathbb{X}_{t}}\right)$ is constant. These assumptions are satisfied when $X$ is either a K3 surface or a product of K3 surfaces.

Fix $n>0$ and fix an embedding $\operatorname{Spec}\left(R_{n}\right) \subset D$ choosing a local parameter $t$ around $0 \in D$ and define $\pi_{n}:=\left.\pi\right|_{\mathcal{X}_{n}}: \mathcal{X}_{n}:=\mathbb{X} \times_{D} \operatorname{Spec}\left(R_{n}\right) \rightarrow \operatorname{Spec}\left(R_{n}\right)$. Let $\kappa_{n-1} \in \operatorname{Ext}_{\mathcal{X}_{n-1}}^{1}\left(\Omega_{\pi_{n-1}}, \mathcal{O}_{\mathcal{X}_{n-1}}\right)$ be the 
relative Kodaira-Spencer class and suppose that $\beta \in \operatorname{Ext}_{\mathcal{X}_{n}}^{1}\left(\Omega_{\pi_{n}}, \mathcal{O}_{\mathcal{X}_{n}}\right)$ is a lift of $\kappa_{n-1}$ under the natural restriction map,

$$
\operatorname{Ext}_{\mathcal{X}_{n}}^{1}\left(\Omega_{\pi_{n}}, \mathcal{O}_{\mathcal{X}_{n}}\right) \longrightarrow \operatorname{Ext}_{\mathcal{X}_{n-1}}^{1}\left(\Omega_{\pi_{n-1}}, \mathcal{O}_{\mathcal{X}_{n-1}}\right), \quad \beta \longmapsto \kappa_{n-1} .
$$

Then $\operatorname{Spec}\left(R_{n}\right) \subset D$ can be extended to $\operatorname{Spec}\left(R_{n+1}\right) \subset D$ such that $\beta=\kappa_{n}$, i.e. $\beta$ is the relative Kodaira-Spencer class on $\mathcal{X}_{n}$ determined by $\mathcal{X}_{n+1}:=\mathbb{X} \times_{D} \operatorname{Spec}\left(R_{n+1}\right)$. Indeed, $\beta$ considered as a section of $\left.\left.\operatorname{Ext}_{\mathcal{X}_{n}}^{1}\left(\Omega_{\pi_{n}}, \mathcal{O}_{\mathcal{X}_{n}}\right) \simeq \mathcal{E} x t_{\pi}^{1}\left(\Omega_{\pi}, \mathcal{O}_{\mathbb{X}}\right)\right|_{\operatorname{Spec}\left(R_{n}\right)} \simeq \mathcal{T}_{D}\right|_{\operatorname{Spec}\left(R_{n}\right)}$ can be locally extended to a vector field on $D$. Integrating this vector field yields a smooth curve $S \subset D$ containing $\operatorname{Spec}\left(R_{n}\right)$. The image of the restriction $\left.\mathcal{T}_{S}\right|_{\operatorname{Spec}\left(R_{n}\right)} \rightarrow \mathcal{E} x t_{\pi_{n}}^{1}\left(\Omega_{\pi_{n}}, \mathcal{O}_{\mathcal{X}_{n}}\right)$ of the Kodaira-Spencer map $\mathcal{T}_{S} \longrightarrow \mathcal{E} x t_{\pi_{S}}^{1}\left(\Omega_{\pi_{S}}, \mathcal{O}_{\mathbb{X}_{S}}\right)$ is thus spanned by $\beta$. Choosing the embedding $\operatorname{Spec}\left(R_{n+1}\right)$ (i.e. the local parameter) appropriately, one can assume that $\beta=\kappa_{n}$.

Later we will consider two situations. We shall start with a deformation over a smooth onedimensional base and study the induced finite order and formal neighbourhoods. This information will be used to construct an a priori different formal deformation by describing recursively the relative Kodaira-Spencer classes of arbitrary order.

\section{Deformation of Derived equivalences of K3 Surfaces}

Let $X$ and $X^{\prime}$ be two projective K3 surfaces and let

$$
\Phi_{\mathcal{E}_{0}}: \mathrm{D}^{\mathrm{b}}(X) \stackrel{\sim}{\longrightarrow} \mathrm{D}^{\mathrm{b}}\left(X^{\prime}\right)
$$

be a Fourier-Mukai equivalence with kernel $\mathcal{E}_{0} \in \mathrm{D}^{\mathrm{b}}\left(X \times X^{\prime}\right)$. For most of Section 4 we will only consider the case $X=X^{\prime}$. In order to distinguish both sides of the Fourier-Mukai equivalence however, we will nevertheless use $X^{\prime}$ for the right hand side.

In this section we complete (see end of Section 4.4) the proof of our main result, which we restate here in a different form.

Theorem 4.1. Suppose $X=X^{\prime}$. Then the induced Hodge isometry $\Phi_{\mathcal{E}_{0}}^{H^{*}}: \widetilde{H}(X, \mathbb{Z}) \stackrel{\sim}{\longrightarrow} \widetilde{H}(X, \mathbb{Z})$ satisfies

$$
\Phi_{\mathcal{E}_{0}}^{H^{*}} \neq\left(-\mathrm{id}_{H^{2}}\right) \oplus \mathrm{id}_{H^{0} \oplus H^{4}} .
$$

As all orientation preserving Hodge isometries do lift to autoequivalences (see [15, 21, 31] or [17, Ch. 10]), this seemingly weaker form is equivalent to the original Theorem 2.

The proof splits in several steps and we argue by contradiction. First, we need to translate the hypothesis, which is in terms of singular cohomology, into the language of Hochschild homology. This will allow us to deform the given Fourier-Mukai kernel sideways to first order (see Section 4.1). Extending the kernel to arbitrary order is more involved, it will take up Section 4.2. Using results of Lieblich, we conclude in Section 4.3 that the Fourier-Mukai kernel can be extended to a perfect complex on the formal scheme and thus leads to a derived equivalence of the general fibres. The kernel of any Fourier-Mukai equivalence of the general fibre however has been shown in Section 2.7 to be a sheaf. In Section 4.4 we explain how this leads to a contradiction when going back to the special fibre.

\subsection{From singular cohomology to first order obstruction. Suppose}

$$
\Phi_{\mathcal{E}_{0}}^{H^{*}}: \widetilde{H}(X, \mathbb{Z}) \stackrel{\sim}{\longrightarrow} \widetilde{H}\left(X^{\prime}, \mathbb{Z}\right)
$$

preserves the Kähler cone up to sign, i.e. $\Phi_{\mathcal{E}_{0}}^{H^{*}}\left(\mathcal{K}_{X}\right)= \pm \mathcal{K}_{X^{\prime}}$. In the situation of our main theorem we will have $X=X^{\prime}$ and $\Phi_{\mathcal{E}_{0}}^{H^{*}}$ acts on $H^{2}(X, \mathbb{Z})$ by -id and thus indeed $\Phi_{\mathcal{E}_{0}}^{H^{2}}\left(\mathcal{K}_{X}\right)=-\mathcal{K}_{X}$.

Consider a real ample class $\omega$ on $X$, i.e. $\omega \in \mathcal{K}_{X} \cap(\operatorname{Pic}(X) \otimes \mathbb{R})$ and let $v_{0} \in H^{1}\left(X, \mathcal{T}_{X}\right)$ be the Kodaira-Spencer class of the first order deformation of $X$ given by the twistor space $\mathbb{X}(\omega) \rightarrow \mathbb{P}(\omega)$ associated to the Kähler class $\omega$. More precisely, up to scaling, $v_{0}$ maps to $\omega$ under the isomorphism $H^{1}\left(X, \mathcal{T}_{X}\right) \stackrel{\sim}{\longrightarrow} H^{1}\left(X, \Omega_{X}^{1}\right)$ induced by a fixed trivializing section $\sigma \in H^{0}\left(X, \omega_{X}\right)$. 
Lemma 4.2. Under the above assumptions, $v_{0}^{\prime}:=\Phi_{\mathcal{E}_{0}}^{\mathrm{HT}^{*}}\left(v_{0}\right) \in H^{1}\left(X^{\prime}, \mathcal{T}_{X^{\prime}}\right) \subset \operatorname{HT}^{2}\left(X^{\prime}\right)$.

Proof. Since $\omega$ can be written as a real linear combination of integral ample classes and all isomorphisms are linear, it suffices to prove the assertion for $\omega=c_{1}(L)$ with $L$ an ample line bundle. Then there exists a line bundle $L^{\prime} \in \operatorname{Pic}\left(X^{\prime}\right)$ such that $\Phi_{\mathcal{E}_{0}}^{H^{*}}\left(c_{1}(L)\right)=\mathrm{c}_{1}\left(L^{\prime}\right)$. We claim that then also $\Phi_{\mathcal{E}_{0}}^{\mathrm{H} \Omega_{*}}\left(c_{1}(L)\right)=\mathrm{c}_{1}\left(L^{\prime}\right)$. Indeed, by Lemma 3.5, ii), one knows that $\Phi_{\mathcal{E}_{0}}^{\mathrm{HH}_{*}} \circ \mathrm{ch}^{\mathrm{HH}_{*}}=\mathrm{ch}^{\mathrm{HH}} \circ \Phi_{\mathcal{E}_{0}}$, which combined with (3.5) yields $\Phi^{\mathrm{H} \Omega_{*}} \circ \mathrm{ch}=\operatorname{ch} \circ \Phi_{\mathcal{E}_{0}}$. On the other hand, for K3 surfaces one has $\operatorname{td}\left(X^{\prime}\right)^{1 / 2} \cdot\left(\operatorname{ch} \circ \Phi_{\mathcal{E}_{0}}\right)=v \circ \Phi_{\mathcal{E}_{0}}=\Phi_{\mathcal{E}_{0}}^{H^{*}} \circ v$, where $v:=\operatorname{td}^{1 / 2} \cdot \operatorname{ch}$ is the Mukai vector on $X$ respectively $X^{\prime}$. Thus, $\operatorname{td}\left(X^{\prime}\right)^{1 / 2} \cdot\left(\Phi_{\mathcal{E}_{0}}^{\mathrm{H} \Omega_{*}} \circ \mathrm{ch}\right)=\operatorname{td}\left(X^{\prime}\right)^{1 / 2} \cdot\left(\operatorname{ch} \circ \Phi_{\mathcal{E}_{0}}\right)=\Phi_{\mathcal{E}_{0}}^{H^{*}} \circ v$. Since multiplication with $\operatorname{td}^{1 / 2}$ does not affect the component of degree two, this shows that $\Phi_{\mathcal{E}_{0}}^{H^{*}}\left(\mathrm{c}_{1}(L)\right)=\mathrm{c}_{1}\left(L^{\prime}\right)$ implies $\Phi_{\mathcal{E}_{0}}^{\mathrm{H} \Omega_{*}}\left(\mathrm{c}_{1}(L)\right)=\mathrm{c}_{1}\left(L^{\prime}\right)$.

In the next step we shall use the following:

Claim. Suppose $\alpha \in \mathrm{HH}_{0}$ with $I(\alpha) \in H^{1}(\Omega) \subset \mathrm{H} \Omega_{0}$. Let $w \in \mathrm{HH}^{2}$ such that $w \cdot \sigma=\alpha$ and $w_{0}:=I(w) \in \mathrm{HT}^{2}$. Then

$$
\left.w_{0}\right\lrcorner \sigma=I(\alpha) .
$$

Indeed, $\left.\left.\left.w_{0}\right\lrcorner \sigma=I(w)\right\lrcorner I(\sigma)=\left(\operatorname{td}^{-1 / 2} I(w)\right)\right\lrcorner\left(\operatorname{td}^{1 / 2} I(\sigma)\right)=\operatorname{td}^{1 / 2} I(w \cdot \sigma)=I(\alpha)$. Here we used $I(\alpha) \in H^{1}\left(\Omega^{1}\right)$ for the second and the last equality (write down the bidegree decomposition for $I(w)$ which a priori might have components not contained in $\left.H^{1}(\mathcal{T})\right), \sigma \in H^{0}\left(\Omega^{2}\right)$ for the second one, and [26, Thm. 1.2] for the penultimate one.

As $\mathrm{c}_{1}(L) \in H^{1}(\Omega)$, by the previous claim, there exists $w_{0} \in \mathrm{HT}^{2}$ such that $\left.\mathrm{c}_{1}(L)=w_{0}\right\lrcorner \sigma$. Hence, if $\sigma^{\prime}:=\Phi_{\mathcal{E}_{0}}^{\mathrm{H} \Omega_{*}}(\sigma)$, we get the following sequence of equalities:

$$
\begin{aligned}
\Phi^{\mathrm{H} \Omega_{*}}\left(\mathrm{c}_{1}(L)\right) & \left.\left.=\Phi^{\mathrm{H} \Omega_{*}}\left(w_{0}\right\lrcorner \sigma\right)=I \Phi^{\mathrm{HH}_{*}} I^{-1}\left(w_{0}\right\lrcorner \sigma\right) \\
& \stackrel{(\mathrm{i})}{=} I \Phi^{\mathrm{HH}_{*}}(w \cdot \sigma) \stackrel{(\mathrm{ii})}{=} I\left(\Phi^{\mathrm{HH}^{*}}(w) \cdot \sigma^{\prime}\right) \\
& \left.\stackrel{(\mathrm{iii})}{=}\left(I \Phi^{\mathrm{HH}}(w)\right)\right\lrcorner \sigma^{\prime},
\end{aligned}
$$

where (i) follows from the claim for $I(w)=w_{0}$, (ii) is due to the multiplicativity of $\left(\Phi^{\mathrm{HH}^{*}}, \Phi^{\mathrm{HH}_{*}}\right)$, and (iii) is obtained by applying again the claim to the $(1,1)$-class $c_{1}\left(L^{\prime}\right)=\Phi_{\mathcal{E}_{0}}^{\mathrm{H} \Omega_{*}}\left(c_{1}(L)\right)$. Thus, $\left.\left(I \Phi^{\mathrm{HH}^{*}}(w)\right)\right\lrcorner \sigma^{\prime} \in H^{1,1}\left(X^{\prime}\right)$, which suffices to conclude $v_{0}^{\prime} \in H^{1}\left(X^{\prime}, \mathcal{T}_{X^{\prime}}\right)$.

Remark 4.3. The result in Lemma 4.2 can be derived from the compatibility between the action of a Fourier-Mukai transform on Hochschild homology and the one on singular cohomology proved in [27, Thm. 1.2] which, in turn, relies on [28, 32]. The main result in [32] can also be used to deduce directly (4.2).

Using for example [34, we can then construct $\mathcal{X}_{1}^{\prime} \rightarrow \operatorname{Spec}\left(R_{1}\right)$ with Kodaira-Spencer class $v_{0}^{\prime} \in H^{1}\left(X^{\prime}, \mathcal{T}_{X^{\prime}}\right)$. Note that by construction $\mathcal{X}_{1}^{\prime} \rightarrow \operatorname{Spec}\left(R_{1}\right)$ depends on the actual FourierMukai kernel $\mathcal{E}_{0}$.

Corollary 4.4. The Fourier-Mukai kernel $\mathcal{E}_{0}$ extends to a perfect complex $\mathcal{E}_{1} \in \mathrm{D}_{\text {perf }}\left(\mathcal{X}_{1} \times_{R_{1}} \mathcal{X}_{1}^{\prime}\right)$ inducing an equivalence $\Phi_{\mathcal{E}_{1}}: \mathrm{D}^{\mathrm{b}}\left(\mathcal{X}_{1}\right) \stackrel{\sim}{\longrightarrow} \mathrm{D}^{\mathrm{b}}\left(\mathcal{X}_{1}^{\prime}\right)$.

Proof. As $\mathcal{E}_{0}$ is the Fourier-Mukai kernel of an equivalence, $\mathcal{O}_{\Delta} \otimes \mathcal{E}_{0}$ defines an equivalence $\mathrm{D}^{\mathrm{b}}(X \times X) \simeq \mathrm{D}^{\mathrm{b}}\left(X \times X^{\prime}\right)$ that sends $\mathcal{O}_{\Delta}$ to $\mathcal{E}_{0}$. In particular, this yields an isomorphism $\operatorname{Ext}_{X \times X}^{1}\left(\mathcal{O}_{\Delta}, \mathcal{O}_{\Delta}\right) \simeq \operatorname{Ext}_{X \times X^{\prime}}^{1}\left(\mathcal{E}_{0}, \mathcal{E}_{0}\right)$. But since $\operatorname{Ext}_{X \times X}^{1}\left(\mathcal{O}_{\Delta}, \mathcal{O}_{\Delta}\right) \simeq H^{0}\left(X, \mathcal{T}_{X}\right) \oplus H^{1}\left(X, \mathcal{O}_{X}\right)=0$ for the K3 surface $X$, this immediately shows that $\mathcal{E}_{0}$ is rigid. Hence the existence of $\mathcal{E}_{1}$ follows from Proposition 3.7. The assertion that $\Phi_{\mathcal{E}_{1}}$ is again an equivalence is part iii) of Remark 2.3. 
4.2. Deforming to higher order. The idea to proceed is to extend recursively $\mathcal{X}_{n}^{\prime}$ to $\mathcal{X}_{n+1}^{\prime}$ such that the Fourier-Mukai kernel $\mathcal{E}_{n}$ on $\mathcal{X}_{n} \times_{R_{n}} \mathcal{X}_{n}^{\prime}$ deforms to a perfect complex on $\mathcal{X}_{n+1} \times_{R_{n+1}} \mathcal{X}_{n+1}^{\prime}$.

We will choose a formal twistor space $\pi: \mathcal{X} \rightarrow \operatorname{Spf}(R)$ of $X$ associated to a very general real ample class $\omega$ with $n$-th order neighbourhoods $\pi_{n}: \mathcal{X}_{n} \rightarrow \operatorname{Spec}\left(R_{n}\right)$. The relative Kodaira-Spencer classes of $\mathcal{X}_{n} \subset \mathcal{X}_{n+1}$ will be denoted $v_{n} \in H^{1}\left(\mathcal{X}_{n}, \mathcal{T}_{\pi_{n}}\right)$.

Suppose we have constructed $\pi_{n}^{\prime}: \mathcal{X}_{n}^{\prime} \rightarrow \operatorname{Spec}\left(R_{n}\right)$ and a perfect complex $\mathcal{E}_{n} \in \mathrm{D}_{\text {perf }}\left(\mathcal{X}_{n} \times_{R_{n}} \mathcal{X}_{n}^{\prime}\right)$ such that $\Phi:=\Phi_{\mathcal{E}_{n}}: \mathrm{D}^{\mathrm{b}}\left(\mathcal{X}_{n}\right) \stackrel{\sim}{\longrightarrow} \mathrm{D}^{\mathrm{b}}\left(\mathcal{X}_{n}^{\prime}\right)$ is an equivalence. Then let

$$
v_{n}^{\prime}:=\Phi^{\mathrm{HT}^{2}}\left(v_{n}\right) \in \operatorname{HT}^{2}\left(\mathcal{X}_{n}^{\prime} / R_{n}\right) .
$$

We would like to view $v_{n}^{\prime}$ as a relative Kodaira-Spencer class of order $n$ on $\mathcal{X}_{n}^{\prime}$ of some extension $\mathcal{X}_{n}^{\prime} \subset \mathcal{X}_{n+1}^{\prime} \longrightarrow \operatorname{Spec}\left(R_{n+1}\right)$. For this we need the following lemma, which is the higher order version of Lemma 4.2. However, the reader will observe that the arguments in the two situations are different and neither of the proofs can be adapted to cover the other case as well.

Lemma 4.5. The class $v_{n}^{\prime}$ is contained in $H^{1}\left(\mathcal{X}_{n}^{\prime}, \mathcal{T}_{\pi_{n}^{\prime}}\right) \subset \operatorname{HT}^{2}\left(\mathcal{X}_{n}^{\prime} / R_{n}\right)$.

Proof. Let $\sigma_{n} \in \mathrm{HH}_{2}\left(\mathcal{X}_{n} / R_{n}\right)=H^{0}\left(\mathcal{X}_{n}, \omega_{\pi_{n}}\right)=\mathrm{H} \Omega_{2}\left(\mathcal{X}_{n} / R_{n}\right)$ be a trivializing section of $\omega_{\pi_{n}}$ and let $\sigma_{n}^{\prime}:= \pm \Phi^{\mathrm{H} \Omega_{*}}\left(\sigma_{n}\right) \in \mathrm{HH}_{2}\left(\mathcal{X}_{n}^{\prime} / R_{n}\right)$. Furthermore, let $\left.\omega_{n}:=v_{n}\right\lrcorner \sigma_{n} \in H^{1}\left(\mathcal{X}_{n}, \Omega_{\pi_{n}}\right) \subset \mathrm{H} \Omega_{0}\left(\mathcal{X}_{n} / R_{n}\right)$ and $\left.\omega_{n}^{\prime}:=v_{n}^{\prime}\right\lrcorner \sigma_{n}^{\prime} \in \mathrm{H} \Omega_{0}\left(\mathcal{X}_{n}^{\prime} / R_{n}\right)$. Then also $\omega_{n}^{\prime}= \pm \Phi^{\mathrm{H} \Omega_{0}}\left(\omega_{n}\right)$, as $\left(\Phi^{\mathrm{HH}^{*}}, \Phi^{\mathrm{HH}_{*}}\right)$ is compatible with the multiplicative structure.

Clearly, $v_{n}^{\prime}$ is contained in $H^{1}\left(\mathcal{X}_{n}^{\prime}, \mathcal{T}_{\pi_{n}^{\prime}}\right) \subset \operatorname{HT}^{2}\left(\mathcal{X}_{n}^{\prime} / R_{n}\right)$ if and only if $\omega_{n}^{\prime}$ is contained in $H^{1}\left(\mathcal{X}_{n}^{\prime}, \Omega_{\pi_{n}^{\prime}}\right) \subset \mathrm{H} \Omega_{0}\left(\mathcal{X}_{n}^{\prime} / R_{n}\right)$.

In a first step, we shall show that $\Phi^{\mathrm{H} \Omega_{0}}: \mathrm{H} \Omega_{0}\left(\mathcal{X}_{n} / R_{n}\right) \stackrel{\sim}{\longrightarrow} \mathrm{H} \Omega_{0}\left(\mathcal{X}_{n}^{\prime} / R_{n}\right)$ preserves $H^{0,0} \oplus H^{2,2}$, i.e. that it maps $\left(H^{0,0} \oplus H^{2,2}\right)\left(\mathcal{X}_{n} / R_{n}\right):=H^{0}\left(\mathcal{X}_{n}, \mathcal{O}_{\mathcal{X}_{n}}\right) \oplus H^{2}\left(\mathcal{X}_{n}, \omega_{\pi_{n}}\right)$ to $\left(H^{0,0} \oplus H^{2,2}\right)\left(\mathcal{X}_{n}^{\prime} / R_{n}\right):=$ $H^{0}\left(\mathcal{X}_{n}^{\prime}, \mathcal{O}_{\mathcal{X}_{n}^{\prime}}\right) \oplus H^{2}\left(\mathcal{X}_{n}^{\prime}, \omega_{\pi_{n}^{\prime}}\right)$.

To this end, consider the Chern character $\operatorname{ch}\left(E_{n}\right) \in \mathrm{H} \Omega_{0}\left(\mathcal{X}_{n} / R_{n}\right)$ for arbitrary $E_{n} \in \mathrm{D}_{\text {perf }}\left(\mathcal{X}_{n}\right)$. In particular, $\operatorname{ch}\left(\mathcal{O}_{\mathcal{X}_{n}}\right)=1 \in H^{0}\left(\mathcal{X}_{n}, \mathcal{O}_{\mathcal{X}_{n}}\right) \subset \mathrm{H} \Omega_{0}\left(\mathcal{X}_{n} / R_{n}\right)$, since $A\left(\mathcal{O}_{\mathcal{X}_{n}}\right)$ is by definition trivial. Furthermore, if $k\left(x_{n}\right) \in \mathrm{D}^{\mathrm{b}}\left(\mathcal{X}_{n}\right)$ denotes the structure sheaf of a section of $\pi_{n}: \mathcal{X}_{n} \rightarrow \operatorname{Spec}\left(R_{n}\right)$, then $\operatorname{ch}\left(k\left(x_{n}\right)\right)$ is contained in $H^{2}\left(\mathcal{X}_{n}, \omega_{\pi_{n}}\right) \subset \mathrm{H} \Omega_{0}\left(\mathcal{X}_{n} / R_{n}\right)$, as rank and determinant of $k\left(x_{n}\right)$ are trivial. Actually, $\operatorname{ch}\left(k\left(x_{n}\right)\right)$ trivializes the $R_{n}$-module $H^{2}\left(\mathcal{X}_{n}, \omega_{\pi_{n}}\right)$. Indeed, since the Atiyah class is compatible with pull-back, one has $j_{n}^{*} \operatorname{ch}\left(k\left(x_{n}\right)\right)=\operatorname{ch}\left(k\left(x_{0}\right)\right)$ and the latter is clearly non-trivial in $H^{2,2}(X)=\mathbb{C}$.

So, $\left(H^{0,0} \oplus H^{2,2}\right)\left(\mathcal{X}_{n} / R_{n}\right)$ is contained in the $R_{n}$-submodule of $\mathrm{H} \Omega_{0}\left(\mathcal{X}_{n} / R_{n}\right)$ spanned by the Chern character of perfect complexes. The analogous assertion holds true for $\mathcal{X}_{n}^{\prime}$.

As we will show now, in fact equality holds. This will later be needed only for $\mathcal{X}_{n}^{\prime}$. So we write it down in this case. If $E_{n}^{\prime} \in \mathrm{D}_{\text {perf }}\left(\mathcal{X}_{n}^{\prime}\right)$, then $\operatorname{ch}_{1}\left(E_{n}^{\prime}\right) \in H^{1}\left(\mathcal{X}_{n}, \Omega_{\pi_{n}^{\prime}}\right)$ equals $\operatorname{tr}\left(A\left(E_{n}^{\prime}\right)\right)$, which by standard arguments is simply $A\left(\operatorname{det}\left(E_{n}^{\prime}\right)\right)$. The $\operatorname{determinant} \operatorname{det}\left(E_{n}^{\prime}\right)$ is a line bundle on $\mathcal{X}_{n}^{\prime}$. Therefore, it suffices to prove that any line bundle on $\mathcal{X}_{n}^{\prime}$ is trivial, but this has been discussed already in Section 2.4. In fact, it suffices to prove this for $n=1$ and then $\mathcal{X}_{1}^{\prime} \rightarrow \operatorname{Spec}\left(R_{1}\right)$ is the first infinitesimal neighbourhood of $X^{\prime}$ inside its twistor space associated to the Kähler class $\omega^{\prime}$ (see Remark 2.13).

As explained in the proof of Lemma 4.2, (3.5) and part ii) of Lemma 3.5 imply $\Phi^{\mathrm{H} \Omega_{0}} \circ \mathrm{ch}=\operatorname{ch} \circ \Phi$. Hence $\Phi^{\mathrm{H} \Omega_{0}}\left(\operatorname{ch}\left(E_{n}\right)\right)$ is contained in $\left(H^{0,0} \oplus H^{2,2}\right)\left(\mathcal{X}_{n}^{\prime} / R_{n}\right)$ for any $E_{n} \in \mathrm{D}_{\text {perf }}\left(\mathcal{X}_{n}\right)$ and therefore

$$
\Phi^{\mathrm{H} \Omega_{0}}\left(\left(H^{0,0} \oplus H^{2,2}\right)\left(\mathcal{X}_{n} / R_{n}\right)\right) \subset\left(H^{0,0} \oplus H^{2,2}\right)\left(\mathcal{X}_{n}^{\prime} / R_{n}\right) .
$$

Let now $w_{n}:=I^{-1}\left(v_{n}\right) \in \mathrm{HH}^{2}\left(\mathcal{X}_{n} / R_{n}\right)$ and $w_{n}^{\prime}:=I^{-1}\left(v_{n}^{\prime}\right) \in \mathrm{HH}^{2}\left(\mathcal{X}_{n}^{\prime} / R_{n}\right)$. Then by definition of $\Phi^{\mathrm{HT}^{*}}$ we have $\Phi^{\mathrm{HH}^{2}}\left(w_{n}\right)=w_{n}^{\prime}$. The multiplicativity of $\left(\Phi^{\mathrm{HH}^{*}}, \Phi^{\mathrm{HH}_{*}}\right)$ and Lemma 3.5. ii) imply $\Phi^{\mathrm{HH}_{*}}\left(w_{n} \cdot \operatorname{ch}^{\mathrm{HH}_{*}}\left(E_{n}\right)\right)=w_{n}^{\prime} \cdot \operatorname{ch}^{\mathrm{HH}_{*}}\left(\Phi\left(E_{n}\right)\right)$. So, $w_{n} \cdot \operatorname{ch}^{\mathrm{HH}_{*}}\left(E_{n}\right)=0$ for all $E_{n} \in \mathrm{D}_{\text {perf }}\left(\mathcal{X}_{n}\right)$ if and only if $w_{n}^{\prime} \cdot \operatorname{ch}^{\mathrm{HH}_{*}}\left(E_{n}^{\prime}\right)=0$ for all $E_{n}^{\prime} \in \mathrm{D}_{\text {perf }}\left(\mathcal{X}_{n}^{\prime}\right)$. 
Suppose we know already that in general

$$
\left.w_{n} \cdot \operatorname{ch}^{\mathrm{HH}_{*}}\left(E_{n}\right)=0 \text { if and only if } v_{n}\right\lrcorner \operatorname{ch}\left(E_{n}\right)=0
$$

and the analogous statement on $\mathcal{X}_{n}^{\prime}$. (For this assertion our assumptions that $\mathcal{X}_{n} \rightarrow \operatorname{Spec}\left(R_{n}\right)$ is of dimension two and that $v_{n}=I\left(w_{n}\right) \in \mathrm{HT}^{2}\left(\mathcal{X}_{n} / R_{n}\right), w_{n} \in \mathrm{HH}^{2}\left(\mathcal{X}_{n} / R_{n}\right)$ are important. See below. $)$ Then one concludes as follows. Since obviously $\left.v_{n}\right\lrcorner\left(H^{0,0} \oplus H^{2,2}\right)\left(\mathcal{X}_{n} / R_{n}\right)=0$ and as shown above $\operatorname{Im}(\mathrm{ch}) \subset\left(H^{0,0} \oplus H^{2,2}\right)\left(\mathcal{X}_{n} / R_{n}\right)$, the 'if' direction in (4.4) would yield $w_{n} \cdot \mathrm{ch}^{\mathrm{HH}_{*}}\left(E_{n}\right)=0$ for all $E_{n} \in$ $\mathrm{D}_{\text {perf }}\left(\mathcal{X}_{n}\right)$ and hence $w_{n}^{\prime} \cdot \mathrm{ch}^{\mathrm{HH}_{*}}\left(E_{n}^{\prime}\right)=0$ for all $E_{n}^{\prime} \in \mathrm{D}_{\text {perf }}\left(\mathcal{X}_{n}^{\prime}\right)$. As $\left(H^{0,0} \oplus H^{2,2}\right)\left(\mathcal{X}_{n}^{\prime} / R_{n}\right)$ is actually spanned by $\operatorname{Im}(\mathrm{ch})$, the 'only if' direction in (4.4) then shows that $\left.v_{n}^{\prime}\right\lrcorner\left(H^{0,0} \oplus H^{2,2}\right)\left(\mathcal{X}_{n}^{\prime} / R_{n}\right)=0$. The latter clearly means $v_{n}^{\prime} \in H^{1}\left(\mathcal{X}_{n}^{\prime}, \mathcal{T}_{\pi_{n}^{\prime}}\right)$.

The assertion (4.4) follows almost directly from (3.4). More precisely, in our situation, 5, Cor. 5.2.3] says that for any $v_{n} \in \operatorname{HT}^{2}\left(\mathcal{X}_{n} / R_{n}\right)$ and any $E_{n} \in \mathrm{D}_{\text {perf }}\left(\mathcal{X}_{n}\right)$ the part of $\left.v_{n}\right\lrcorner\left(\exp \left(A\left(\mathcal{F}_{n}\right)\right)\right)$ contained in $\operatorname{Ext}_{\mathcal{X}_{n}}^{2}\left(E_{n}, E_{n}\right)$ coincides with the projection of $I^{-1}\left(v_{n}\right) \cdot A H\left(E_{n}\right)$ under $\operatorname{Ext}_{\mathcal{X}_{n}}^{2}\left(E_{n}, E_{n} \otimes\right.$ $\left.\eta_{n}^{*} \mathcal{O}_{\Delta_{n}}\right) \stackrel{\sim}{\longrightarrow} \operatorname{Ext}_{\mathcal{X}_{n}}^{2}\left(E_{n}, E_{n}\right)$. Taking traces on both sides yields $\left.v_{n}\right\lrcorner \operatorname{ch}\left(E_{n}\right)=I^{-1}\left(v_{n}\right) \cdot \operatorname{ch}^{\mathrm{HH}_{*}}\left(E_{n}\right)$, which then proves (4.4).

Corollary 4.6. If $\mathcal{E}_{n} \in \mathrm{D}_{\text {perf }}\left(\mathcal{X}_{n} \times_{R_{n}} \mathcal{X}_{n}^{\prime}\right)$ induces an equivalences $\Phi_{\mathcal{E}_{n}}: \mathrm{D}^{\mathrm{b}}\left(\mathcal{X}_{n}\right) \stackrel{\sim}{\longrightarrow} \mathrm{D}^{\mathrm{b}}\left(\mathcal{X}_{n}^{\prime}\right)$, then there exists a deformation $\mathcal{X}_{n+1}^{\prime} \rightarrow \operatorname{Spec}\left(R_{n+1}\right)$ of $\mathcal{X}_{n}^{\prime} \rightarrow \operatorname{Spec}\left(R_{n}\right)$ and a complex $\mathcal{E}_{n+1} \in$ $\mathrm{D}_{\text {perf }}\left(\mathcal{X}_{n+1} \times_{R_{n+1}} \mathcal{X}_{n+1}^{\prime}\right)$ such that $\mathcal{E}_{n} \simeq L i_{n}^{*} \mathcal{E}_{n+1}$. Moreover, $\mathcal{E}_{n+1}$ induces an equivalence $\Phi_{\mathcal{E}_{n+1}}$ : $\mathrm{D}^{\mathrm{b}}\left(\mathcal{X}_{n+1}\right) \stackrel{\sim}{\longrightarrow} \mathrm{D}^{\mathrm{b}}\left(\mathcal{X}_{n+1}^{\prime}\right)$.

Proof. By Example 3.8, choose the extension $\mathcal{X}_{n+1}^{\prime} \rightarrow \operatorname{Spec}\left(R_{n+1}\right)$ such that its Kodaira-Spencer class $\kappa_{n} \in H^{1}\left(\mathcal{X}_{n}^{\prime}, \mathcal{T}_{\pi_{n}^{\prime}}\right)$ is $v_{n}^{\prime}$ in (4.3), which by Lemma 4.5] is indeed an element in $H^{1}\left(\mathcal{X}_{n}^{\prime}, \mathcal{T}_{\pi_{n}^{\prime}}\right)$. Since $\mathcal{E}_{0}=L j_{n}^{*} \mathcal{E}_{n}$ is rigid, Proposition 3.7 allows one to conclude the existence of a complex $\mathcal{E}_{n+1} \in \mathrm{D}_{\text {perf }}\left(\mathcal{X}_{n+1} \times_{R_{n+1}} \mathcal{X}_{n+1}^{\prime}\right)$ with $L i_{n}^{*} \mathcal{E}_{n+1} \simeq \mathcal{E}_{n}$. The last assertion follows from part iii) of Remark 2.3 .

4.3. Deformation to the general fibre. Applying Corollary 4.6 recursively, we obtain a formal scheme $\pi^{\prime}: \mathcal{X}^{\prime} \rightarrow \operatorname{Spf}(R)$ and perfect complexes $\mathcal{E}_{n} \in \mathrm{D}_{\text {perf }}\left(\mathcal{X}_{n} \times_{R_{n}} \mathcal{X}_{n}^{\prime}\right), n \in \mathbb{N}$, inducing FourierMukai equivalences $\Phi_{\mathcal{E}_{n}}: \mathrm{D}^{\mathrm{b}}\left(\mathcal{X}_{n}\right) \stackrel{\sim}{\rightarrow} \mathrm{D}^{\mathrm{b}}\left(\mathcal{X}_{n}^{\prime}\right)$ with $L i_{n}^{*} \mathcal{E}_{n+1} \simeq \mathcal{E}_{n}$ and with $\mathcal{E}_{0}$ as given in (4.1).

Now we use Lieblich's [25, Sect. 3.6] to conclude that the existence of all higher order deformations is enough to show the existence of a formal deformation of the complex. So, there exists a complex $\mathcal{E} \in \mathrm{D}^{\mathrm{b}}\left(\mathcal{X} \times_{R} \mathcal{X}^{\prime}\right)$ with $L \iota_{n}^{*} \mathcal{E} \simeq \mathcal{E}_{n}$, for all $n \in \mathbb{N}$.

Remark 4.7. Lieblich's result is far from being trivial and the proof is quite ingenious. Of course, if a coherent sheaf lifts to any order, it deforms by definition to a sheaf on the formal neighbourhood. For complexes as objects in the derived categories this is a different matter. Note that a priori one really only gets an object in $\mathrm{D}^{\mathrm{b}}\left(\mathcal{X} \times_{R} \mathcal{X}^{\prime}\right)$, which is, by definition, $\mathrm{D}_{\text {coh }}^{\mathrm{b}}\left(\mathcal{O}_{\mathcal{X} \times_{R} \mathcal{X}^{\prime}}-\mathbf{M o d}\right)$, and not in $\mathrm{D}^{\mathrm{b}}\left(\mathbf{C o h}\left(\mathcal{X} \times{ }_{R} \mathcal{X}^{\prime}\right)\right)$ as one could wish for.

For the convenience of the reader, let us recall Lieblich's strategy. Instead of considering deformations of $\mathcal{E}_{0}$ as an object in the derived category, Lieblich shows in [25, Prop. 3.3.4] that by replacing $\mathcal{E}_{0}$ with a complex of quasi-coherent injective sheaves one can work with actual deformations of complexes, i.e. the differentials and objects are deformed (flat over the base) and the restrictions to lower order yield isomorphisms of complexes. By taking limits, one obtains a bounded complex of ind-quasi-coherent sheaves on the formal scheme. Eventually, one has to show that the complex obtained in this way, which is an object in $\mathrm{D}^{\mathrm{b}}\left(\mathcal{O}_{\mathcal{X} \times{ }_{R} \mathcal{X}^{\prime}}\right.$-Mod $)$, has coherent cohomology. This is a local statement and is addressed in [25, Lemma 3.6.11]. Note that the main result [25, Prop. 3.6.1] treats the case that the formal scheme is given as a formal neighbourhood of an actual scheme over $\operatorname{Spec}(R)$ and asserts then the existence of a perfect complex on the scheme. 
In our case, the actual scheme does not exist but only the formal one. However, Lieblich's arguments proving the existence of the perfect complex on the formal scheme, which is the first step in his approach, do not use the existence of the scheme itself.

Now, by Remark 2.3 , iv $), \Phi_{\mathcal{E}_{K}}: \mathrm{D}^{\mathrm{b}}\left(\mathcal{X}_{K}\right) \stackrel{\sim}{\rightarrow} \mathrm{D}^{\mathrm{b}}\left(\mathcal{X}_{K}^{\prime}\right)$ is an equivalence. The Fourier-Mukai equivalence $T_{\mathcal{O}_{\mathcal{X}^{\prime}}}: \mathrm{D}^{\mathrm{b}}\left(\mathcal{X}^{\prime}\right) \stackrel{\sim}{\longrightarrow} \mathrm{D}^{\mathrm{b}}\left(\mathcal{X}^{\prime}\right)$ with kernel $\mathcal{I}_{\Delta_{\mathcal{X}^{\prime}}}[1]$ 'restricted' to the special fibre is the spherical twist

$$
T_{0}: \mathrm{D}^{\mathrm{b}}\left(X^{\prime}\right) \stackrel{\sim}{\longrightarrow} \mathrm{D}^{\mathrm{b}}\left(X^{\prime}\right)
$$

and 'restricted' to the general fibre it yields the spherical twist

$$
T_{K}: \mathrm{D}^{\mathrm{b}}\left(\mathcal{X}_{K}^{\prime}\right) \stackrel{\sim}{\longrightarrow} \mathrm{D}^{\mathrm{b}}\left(\mathcal{X}_{K}^{\prime}\right)
$$

Then Proposition 2.18 asserts that there exist integers $n$ and $m$ such that the composition $T_{K}^{n} \circ$ $\Phi_{\mathcal{E}_{K}}[m]$ defines a bijection between the set of $K$-rational points of $\mathcal{X}_{K}$ and $\mathcal{X}_{K}^{\prime}$. By the discussion in Section 2.7 this is enough to conclude that $T_{K}^{n} \circ \Phi_{\mathcal{E}_{K}}[\mathrm{~m}]$ can be written as a Fourier-Mukai transform whose kernel is a sheaf on $\left(\mathcal{X} \times{ }_{R} \mathcal{X}^{\prime}\right)_{K}$. Note that $n$ and $m$ must both be even. Indeed if a $K$-rational point is sent to a $K$-rational point via $T_{K}^{n} \circ \Phi_{\mathcal{E}_{K}}[m]$, then its restriction to the special fibre $T_{0}^{n} \circ \Phi_{\mathcal{E}_{0}}[m]$ preserves the Mukai vector of a point $(0,0,1)$. Now use that $T_{0}^{H^{*}}$ sends $(0,0,1)$ to $(-1,0,0)$, that the simple shifts acts by $-\mathrm{id}$, and that $\Phi_{\mathcal{E}_{0}}^{H^{*}}$ preserves $(0,0,1)$ by assumption.

The conclusion of the discussion so far is that, up to applying shift and spherical twist, the Fourier-Mukai kernel $\mathcal{E}_{0}$ of an equivalence $\Phi$ with $\Phi^{H^{*}}=-\mathrm{id}_{H^{2}} \oplus \mathrm{id}_{H^{0} \oplus H^{4}}$ deforms to a sheaf on the general fibre $\left(\mathcal{X} \times_{R} \mathcal{X}^{\prime}\right)_{K}$, where $\mathcal{X} \rightarrow \operatorname{Spf}(R)$ is the formal neighbourhood of $X$ inside a very general twistor space and $\mathcal{X}^{\prime} \rightarrow \operatorname{Spf}(R)$ was constructed recursively. One now has to show that this leads to a contradiction.

4.4. Return to the special fibre. Let $X$ be a smooth projective $\mathrm{K} 3$ surface and $\mathcal{G}$ a coherent sheaf on $X \times X$. Consider the Fourier-Mukai transform $\Phi_{\mathcal{G}}: \mathrm{D}^{\mathrm{b}}(X) \rightarrow \mathrm{D}^{\mathrm{b}}(X)$ with kernel $\mathcal{G}$. As we make no further assumptions on $\mathcal{G}, \Phi_{\mathcal{G}}$ is not necessarily an equivalence. We shall be interested in the induced map on cohomology $\Phi_{\mathcal{G}}^{H^{*}}: \widetilde{H}(X, \mathbb{Q}) \longrightarrow \widetilde{H}(X, \mathbb{Q})$.

Lemma 4.8. For any sheaf $\mathcal{G}$ on $X \times X$ one has $\Phi_{\mathcal{G}}^{H^{*}} \neq\left(-\operatorname{id}_{H^{2}}\right) \oplus \operatorname{id}_{H^{0} \oplus H^{4}}$.

Proof. Suppose $\Phi_{\mathcal{G}}^{H^{*}}=\left(-\mathrm{id}_{H^{2}}\right) \oplus \mathrm{id}_{H^{0} \oplus H^{4}}$. Choose an ample line bundle $L$ on $X$. Then for $n, m \gg$ 0 the sheaf $\mathcal{G}_{n, m}:=\mathcal{G} \otimes\left(q^{*} L^{n} \otimes p^{*} L^{m}\right)$ is globally generated and $\Phi_{\mathcal{G}}\left(L^{n}\right)=p_{*}\left(\mathcal{G} \otimes q^{*} L^{n}\right)$ is a sheaf. So, there exists a short exact sequence $0 \longrightarrow \mathcal{K} \longrightarrow \mathcal{O}_{X \times X}^{N} \longrightarrow \mathcal{G}_{n, m} \longrightarrow 0$. Twisting further with $q^{*} L^{n^{\prime}}, n^{\prime} \gg 0$, kills the higher direct images of $\mathcal{K}$ under the projection $p$, i.e. $R^{i} p_{*}\left(\mathcal{K} \otimes q^{*} L^{n^{\prime}}\right)=0$ for $i>0$. Thus, there exists a surjection $\mathcal{O}_{X}^{N^{\prime}} \simeq p_{*}\left(\mathcal{O}_{X \times X}^{N} \otimes q^{*} L^{n^{\prime}}\right) \longrightarrow p_{*}\left(\mathcal{G}_{n+n^{\prime}, m}\right)=\Phi_{\mathcal{G}}\left(L^{n+n^{\prime}}\right) \otimes L^{m}$.

On the other hand, by assumption $v\left(\Phi_{\mathcal{G}}\left(L^{n+n^{\prime}}\right) \otimes L^{m}\right)=1+\left(m-\left(n+n^{\prime}\right)\right) c_{1}(L)+s$ for some $s \in H^{4}(X, \mathbb{Q})$. Thus, $\Phi_{\mathcal{G}}\left(L^{n+n^{\prime}}\right) \otimes L^{m}$ is a globally generated coherent sheaf of rank one with first Chern class $\left(m-\left(n+n^{\prime}\right)\right) \mathrm{c}_{1}(L)$. It is not difficult to see that this is impossible as soon as $m-\left(n+n^{\prime}\right)<0$.

We leave it to the reader to formulate a similar statement for sheaves on the product $X \times X^{\prime}$ of two not necessarily isomorphic K3 surfaces.

Consider two formal deformations $\mathcal{X} \rightarrow \operatorname{Spf}(R)$ and $\mathcal{X}^{\prime} \rightarrow \operatorname{Spf}(R)$ of the same algebraic K3 surface $X=\mathcal{X}_{0}=\mathcal{X}_{0}^{\prime}$.

Corollary 4.9. Let $\mathcal{E} \in \mathrm{D}^{\mathrm{b}}\left(\mathcal{X} \times{ }_{R} \mathcal{X}^{\prime}\right)$ be an object whose restriction to the general fibre is a sheaf, i.e. $\mathcal{E}_{K} \in \mathbf{C o h}\left(\mathcal{X}_{K}\right)$. If $\mathcal{E}_{0} \in \mathrm{D}^{\mathrm{b}}(X \times X)$ denotes the restriction to the special fibre, then the Fourier-Mukai transform $\Phi_{\mathcal{E}_{0}}: \mathrm{D}^{\mathrm{b}}(X) \longrightarrow \mathrm{D}^{\mathrm{b}}(X)$ induces a map $\Phi_{\mathcal{E}_{0}}^{H^{*}}: \widetilde{H}(X, \mathbb{Q}) \longrightarrow \widetilde{H}(X, \mathbb{Q})$ different from $\left(-\mathrm{id}_{H^{2}}\right) \oplus \mathrm{id}_{H^{0} \oplus H^{4}}$. 
Proof. If $\mathcal{E}_{K}$ is a sheaf, then there exists an $R$-flat lift $\tilde{\mathcal{E}} \in \operatorname{Coh}\left(\mathcal{X} \times{ }_{R} \mathcal{X}^{\prime}\right)$ of $\mathcal{E}_{K}$. Thus the complex $\mathcal{E}$ and the sheaf $\tilde{\mathcal{E}}$ coincide on the general fibre or, in other words, they differ by $R$-torsion complexes. In particular, the restrictions to the special fibres $\mathcal{E}_{0}$ and $\tilde{\mathcal{E}}_{0}$ define the same elements in the K-group (see Remark 2.7) and therefore the same correspondence $\Phi_{\mathcal{E}_{0}}^{H^{*}}=\Phi_{\tilde{\mathcal{E}}_{0}}^{H^{*}}: \widetilde{H}(X, \mathbb{Z}) \stackrel{\sim}{\longrightarrow} \widetilde{H}(X, \mathbb{Z})$.

So, $\tilde{\mathcal{E}}_{0}$ is a sheaf(!) on $X \times X$ inducing the same map on cohomology as the complex $\mathcal{E}_{0}$. Now Lemma 4.8 applies and yields the contradiction.

Clearly, the corollary applies directly to our problem with $\mathcal{E}$ as in Section 4.3 and which therefore contradicts the assumption that $\Phi_{\mathcal{E}_{0}}^{H^{*}}$ acts as $\left(-\mathrm{id}_{H^{2}}\right) \oplus \mathrm{id}_{H^{0} \oplus H^{4}}$. This concludes the proof of Theorem 4.1,

4.5. Derived equivalence between non-isomorphic K3 surfaces. The main theorem implies that every derived equivalence between projective K3 surfaces is orientation preserving.

Let $X$ be an arbitrary K3 surface. Then its cohomology $\widetilde{H}(X, \mathbb{Z})$ admits a natural orientation (of the positive directions). Indeed, if $\omega \in H^{1,1}(X)$ is any Kähler class, then $1-\omega^{2} / 2$ and $\omega$ span a positive plane in $\widetilde{H}(X, \mathbb{R})$. Another positive plane orthogonal to it is spanned by real and imaginary part of a generator $\sigma \in H^{2,0}(X)$. Together they span a positive four-space which is endowed with a natural orientation by choosing the base $\operatorname{Re}(\sigma), \operatorname{Im}(\sigma), 1-\omega^{2} / 2, \omega$. This orientation does neither depend on the particular Kähler class $\omega$ nor on the choice of the regular two-form $\sigma$.

If $X^{\prime}$ is another $\mathrm{K} 3$ surface, one says that an isometry $\widetilde{H}(X, \mathbb{Z}) \stackrel{\sim}{\longrightarrow} \widetilde{H}\left(X^{\prime}, \mathbb{Z}\right)$ is orientation preserving if the natural orientations of the four positive directions in $\widetilde{H}(X, \mathbb{R})$ respectively in $\widetilde{H}\left(X^{\prime}, \mathbb{R}\right)$ coincide under the isometry.

Corollary 4.10. Let $\Phi: \mathrm{D}^{\mathrm{b}}(X) \stackrel{\sim}{\longrightarrow} \mathrm{D}^{\mathrm{b}}\left(X^{\prime}\right)$ be an exact equivalence between two projective $K 3$ surfaces. Then the induced Hodge isometry

$$
\Phi^{H^{*}}: \widetilde{H}(X, \mathbb{Z}) \stackrel{\sim}{\longrightarrow} \widetilde{H}\left(X^{\prime}, \mathbb{Z}\right)
$$

is orientation preserving.

Proof. Suppose that $\Phi^{H^{*}}$ is an orientation reversing Hodge isometry. Composing with $-\mathrm{id}_{H^{2}\left(X^{\prime}\right)} \oplus$ $\operatorname{id}_{\left(H^{0} \oplus H^{4}\right)\left(X^{\prime}\right)}$ provides us with an orientation preserving Hodge isometry $\widetilde{H}(X, \mathbb{Z}) \stackrel{\sim}{\longrightarrow} \widetilde{H}\left(X^{\prime}, \mathbb{Z}\right)$. Such a composition can then be lifted to a Fourier-Mukai equivalence $\Psi: \mathrm{D}^{\mathrm{b}}(X) \stackrel{\sim}{\longrightarrow} \mathrm{D}^{\mathrm{b}}\left(X^{\prime}\right)$ (see e.g. [17, Cor. 10.13]). Then

$$
\Psi^{-1} \circ \Phi: \mathrm{D}^{\mathrm{b}}(X) \stackrel{\sim}{\longrightarrow} \mathrm{D}^{\mathrm{b}}(X)
$$

would be an exact equivalence with orientation reversing action on $\widetilde{H}(X, \mathbb{Z})$, which contradicts Theorem 2.

Acknowledgements. We wish to thank M. Lieblich and M. Rapoport for useful discussions and the referees for many insightful comments and suggestions. We gratefully acknowledge the support of the following institutions: Hausdorff Center for Mathematics, IHES, Imperial College, Istituto Nazionale di Alta Matematica, Max-Planck Institute, and SFB/TR 45.

\section{REFERENCES}

[1] A. Beauville, J.-P. Bourguignon, M. Demazure, Géométrie des surfaces K3: modules et périodes, Séminaires Palaiseau. Astérisque 126 (1985).

[2] C. Borcea, Diffeomorphisms of a K3 surface, Math. Ann. 275 (1986), 1-4.

[3] T. Bridgeland, Stability conditions on K3 surfaces, Duke Math. J. 141 (2008), 241-291.

[4] R.-O. Buchweitz, H. Flenner, Global Hochschild (co-)homology of singular spaces, Adv. Math. 217 (2008), 205-242.

[5] R.-O. Buchweitz, H. Flenner, The global decomposition theorem for Hochschild (co-)homology of singular spaces via the Atiyah Chern character, Adv. Math. 217 (2008), 243-281. 
[6] D. Calaque, M. Van den Bergh, Hochschild cohomology and Atiyah classes, arXiv:0708.2725.

[7] A. Căldăraru, The Mukai pairing II: The Hochschild-Kostant-Rosenberg isomorphism, Adv. Math. 194 (2005), $34-66$.

[8] A. Căldăraru, S. Willerton, The Mukai pairing, I: a categorical approach, arXiv:0707.2052.

[9] V. Dolgushev, D. Tamarkin, B. Tsygan, The homotopy Gerstenhaber algebra of Hochschild cochains of a regular algebra is formal, J. Noncommut. Geom. 1 (2007), 1-25.

[10] V. Dolgushev, D. Tamarkin, B. Tsygan, Formality of the homotopy calculus algebra of Hochschild (co)chains, arXiv:0807.5117.

[11] S. Donaldson, Polynomial invariants for smooth four-manifolds, Topology 29 (1990), 257-315.

[12] A. Fujiki, On the de Rham cohomology group of a compact Kähler symplectic manifold, Alg. geom., Sendai, 1985, Adv. Stud. Pure Math. 10 (1987), 105-165.

[13] M. Gross, D. Huybrechts, D. Joyce, Calabi-Yau manifolds and Related Geometries, Universitext, Springer (2003).

[14] R. Hartshorne, Algebraic Geometry, GTM 52, Springer (1977).

[15] S. Hosono, B.H. Lian, K. Oguiso, S.-T. Yau, Autoequivalences of derived category of a K3 surface and monodromy transformations, J. Alg. Geom. 13 (2004), 513-545.

[16] D. Huybrechts, M. Lehn, The geometry of moduli spaces of sheaves, Aspects of Math. E31, Vieweg, Braunschweig, (1997).

[17] D. Huybrechts, Fourier-Mukai transforms in algebraic geometry, Oxford Mathematical Monographs (2006).

[18] D. Huybrechts, E. Macrì, P. Stellari, Stability conditions for generic K3 surfaces, Compositio Math. 144 (2008), $134-162$.

[19] D. Huybrechts, E. Macrì, P. Stellari, Derived equivalences of K3 surfaces and orientation, arXiv:0710.1645v2.

[20] D. Huybrechts, E. Macrì, P. Stellari, Formal deformations and their categorical general fibre, arXiv:0809.3201.

[21] D. Huybrechts, P. Stellari, Equivalences of twisted K3 surfaces, Math. Ann. 332 (2005), 901-936.

[22] D. Huybrechts, R. Thomas, Deformation-obstruction theory for complexes via Atiyah and Kodaira-Spencer classes, arXiv:0805.3527.

[23] L. Illusie, Grothendieck's existence theorem in formal geometry, in Fundamenal Algebraic Geometry, Grothendieck's FGA explained. ed. B. Fantechi et al. Math. Surveys and Mon. 123, AMS (2005).

[24] M. Kontsevich, Deformation quantization of Poisson manifolds, Lett. Math. Phys. 66 (2003), 157-216.

[25] M. Lieblich, Moduli of complexes on a proper morphism, J. Alg. Geom. 15 (2006), 175-206.

[26] E. Macrì, M. Nieper-Wisskirchen, P. Stellari, The module structure of Hochschild homology in some examples, C. R. Acad. Sci. Paris, Ser. I 346 (2008), 863-866.

[27] E. Macrì, P. Stellari, Infinitesimal derived Torelli theorem for K3 surfaces, to appear in: Int. Math. Res. Not., arXiv:0804.2552.

[28] N. Markarian, Poincaré-Birkhoff-Witt isomorphism, Hochschild homology and Riemann-Roch theorem. Preprint MPI 2001-52.

[29] S. Mukai, On the moduli space of bundles on K3 surfaces, I, In: Vector Bundles on Algebraic Varieties, Oxford University Press, Bombay and London (1987), 341-413.

[30] D. Orlov, Equivalences of derived categories and K3 surfaces, J. Math. Sci. 84 (1997), 1361-1381.

[31] D. Ploog, Groups of autoequivalences of derived categories of smooth projective varieties, PhD-thesis, Berlin (2005).

[32] A. Ramadoss, The relative Riemann-Roch theorem from Hochschild homology, New York J. Math. 14 (2008), 643-717..

[33] B. Szendröi, Diffeomorphisms and families of Fourier-Mukai transforms in mirror symmetry, Applications of Alg. Geom. to Coding Theory, Phys. and Comp. NATO Science Series. Kluwer (2001), 317-337.

[34] Y. Toda, Deformations and Fourier-Mukai transforms, J. Diff. Geom. 81 (2009), 197-224.

[35] C. Weibel, Cyclic homology for schemes, Proc. Amer. Math. Soc. 124 (1996), 1655-1662.

[36] A. Yekutieli, The continuous Hochschild cochain complex of a scheme, Canadian J. Math. 54 (2002), $1319-1337$.

D.H.: Mathematisches Institut, Universität Bonn, Beringstr. 1, 53115 Bonn, Germany

E-mail address: huybrech@math.uni-bonn.de

E.M.: Department of Mathematics, University of Utah, 155 South 1400 East, Salt Lake City, UT 84112-0090, USA

E-mail address: macri@math.utah.edu

P.S.: Dipartimento di Matematica "F. Enriques", Università degli Studi di Milano, Via Cesare SALdini 50, 20133 Milano, Italy

E-mail address: paolo.stellari@unimi.it 